\title{
A GENERAL KINETIC ANALYSIS OF NON-LINEAR IRREVERSIBLE COPOLYMERIZATIONS
}

\author{
MÁRIO RUI P. F. N. COSTA and ROLANDO C. S. DIAS \\ LSRE, Faculty of Engineering, University of Porto, Rua dos Bragas, 4099 Porto Codex, Portugal
}

(Received 25 November 1992; accepted for publication 17 August 1993)

\begin{abstract}
Irreversible non-linear copolymerizations with arbitrarily great numbers of monomers are described specifying the stoichiometric coefficients of the reactions involving end-groups. Rate equations for the vectorial generating function or discrete transform of the distribution of end-groups and repeating units and its derivatives with respect to logarithms of Laplace parameters can thus be written. Similar rate equations can be written for the discrete transforms of the distributions of descendants of each linking group with respect to their numbers of end-groups. This allows the prediction of gel properties, such as the concentration of elastically active network chains and mass fraction of pendant material. Mass balance equations using these rate laws are non-linear partial differential equations solvable by the method of characteristics. Numerical methods are developed for computing molecular weight distributions and average molecular weights before and after gelation, as well as the weight fraction of sol and gel properties related to its elasticity behaviour. The curing of a tetraepoxide with a primary diamine is taken as a case study and results are compared with predictions by the theory of branching processes.
\end{abstract}

\section{INTRODUCTION}

This work stems from the kinetic approach for modelling irreversible non-linear polycondensations, developed by Kuchanov and co-workers (Pis'men and Kuchanov, 1971; Kuchanov and Pis'men, 1972a, b; Kuchanov and Povolotskaya, 1982). These earlier works have laid the foundations of a rigorous method for the prediction of average molecular weights, both before and after gelation. However, prediction of gel structure, such as computing the concentrations of elastically active network chains, has remained hitherto an unsolved problem. Moreover, numerical methods for solving the mass balance equations of the kinetic approach have not been implemented, and this has precluded its application to all but the simplest chemical systems.

In this paper, a unified treatment of essentially all irreversible non-linear polymerizations, including polycondensations and polyadditions, is carried out. This is possible because a common set of rules to describe the molecular growth can be established. In order to describe the formation of these polymers, it is necessary to identify the repeating units, which are not changed by chemical reaction, and the end-groups attached to them, which can lead to molecular growth by intermolecular reaction and linking of the units to which they are attached, or become inactivated by a termination or transfer reaction, which may be intramolecular and form a loop.

There is often a large number of those structural constituents which must be distinguished, even with as little as two or three symmetrical monomers at the start of the reaction. This happens because of the following:

(a) Substitution effects, such as the difference in reactivities of the hydroxyls of pentaerythritol after reaction with a carboxylic acid (Gordon and Leonis, 1975a, b); (b) Intramolecular reactions between end-groups, which have a rate depending on the size of the out coming loop, leading inevitably to the consideration of a large number of molecular fragments for their kinetic modelling, even with only the loop with the lowest possible size being allowed to form (Moschiar et al., 1988).

Each time a new kinetic scheme is under study, huge systems of algebraic and differential equations must be written and solved.This is a time-consuming and error-prone task. For instance, a model of phenol-formaldehyde polycondensation (Váquez et al., 1984) requires 19 kinds of end-groups to be defined; the above-cited model of polyurethane formation with consideration of the smallest rings (Moschiar et al., 1988) uses 25 molecular fragments. Still more complex models are likely to be needed.

For that reason, it is desirable that a single computer program could be able to carry out the prediction of polymer properties for any such polymerization scheme, needing only a minimum of usersupplied chemical information, and specially without the need of analytically solving mass balance equations of polymer species. So, the present treatment allows an arbitrary number of monomers and reactive groups, both in polycondensations and polyadditions, with a distribution of molecular weights in monomer classes. The problem of numerical evaluation of molecular weight averages and molecular weight distributions both before and after gelation is completely solved in this contribution, as well as the distribution of the number of cross-links in the gel, allowing therefore an estimation of the concentration of elastically active network chains and other gel properties relevant for the prediction of its elastic properties. Previous uses of the kinetic approach were limited to the prediction of gel-points and average molecular weights in the pre-gel state. 
A GENERAL KINETIC MODEL OF IRREVERSIBLE NON-LINEAR COPOLYMERIZATIONS

Chemical species, their concentrations and molecular weights

Non-alternating copolymerizations, or homopolymerizations of monomers with functionality greater than two, give rise to molecules with a large number of position isomers, except, of course, when the degree of polymerization is very low. For irreversible polymerization reactions, there is no need of distinguishing polymer species according to the way their repeating units are linked, if only properties such as average molecular weights and concentration of active network chains in gel are to be computed.

Let $N$ be the number of monomer classes. Each monomer class contains a chemically invariant moiety (the repeating unit), named $\mathbf{U}^{m}$ for the $m$ th monomer, and a certain number $f_{i}^{m}$ of reactive groups (most often end-groups, sometimes individual molecules), named $\mathbf{A}_{\mathbf{i}}$. The number of chemically distinguishable reacting groups among all monomers will be named $G$. Superscripts will be used in this paper whenever they refer to monomer classes, and subscripts will be introduced when they refer to reactive groups.

For instance, in a polycondensation of adipoyl chloride $\mathrm{ClCO}\left(\mathrm{CH}_{2}\right)_{4} \mathrm{COCl}$ and azelayl chloride $\mathrm{ClCO}\left(\mathrm{CH}_{2}\right)_{5} \mathrm{COCl}$ with amines and/or alcohols, the acid chloride end-groups have virtually identical reactivities (provided intramolecular reactions are negligible), so that the two monomers should be considered as forming a class of difunctional monomers with two $\mathrm{COCl}$ end-groups. If there is caproyl chloride $\mathrm{CH}_{3}\left(\mathrm{CH}_{2}\right)_{4} \mathrm{COCl}$ as a co-monomer, it is necessary to introduce a new class of monofunctional monomers carrying a single $\mathrm{COCl}$ end-group.

Monomer classes are often pre-polymers. For instance, in the formation of a polyurethane, part of the polyol component is usually a polyether or a polyester terminated by two, three or some other number of hydroxyl groups, which have a certain molecular weight distribution. Each repeating unit $\mathbf{U}^{\mathrm{m}}$ has a certain molecular weight distribution, defined by a discrete distribution $\Omega^{m j}$ representing the mole fraction of each subspecies $U^{m j}$ of the monomer class $U^{m}$ with a molecular weight $M^{m j}$. The variable $j$ is any index enumerating the repeating units and going from 0 to $\infty$.

Since there is no need to distinguish among position isomers, it is enough to describe each polymer molecule through the numbers of end-groups and repeating units it contains. So, a molecule with a vector of numbers of end-groups $\mathbf{a}=\left[a_{1} \ldots a_{G}\right]$ and a vector of numbers of repeating units $\mathbf{n}=$ $\left[n^{1} \ldots n^{N}\right]$ shall be named $\mathbf{P}(\mathbf{a}, \mathbf{n})$. Its mole concentration will be written as $P(\mathbf{a}, \mathbf{n})$, in order to simplify as much as possible the equations that follow. This discrete distribution of vectors $\mathbf{a}$ and $\mathbf{n}$ is appropriately named a vector-number-molecular-number distribution (VNMND). It will nearly always be found from its vectorial discrete transform:

$$
\begin{aligned}
\bar{P}(\sigma, \zeta)= & \sum_{a_{1}=0}^{\infty} \ldots \sum_{a_{G}=0}^{\infty} \sum_{n^{1}=0}^{\infty} \ldots \sum_{n^{N}=0}^{\infty} \sigma_{1}^{a_{1}} \ldots \sigma_{G}^{a_{G}}\left(\zeta^{1}\right)^{n^{1}} \\
& \ldots\left(\zeta^{N}\right)^{n^{N}} P\left(a_{1}, \ldots, a_{G}, n^{1}, \ldots, n^{N}\right) .
\end{aligned}
$$

In polyadditions of vinyl compounds, the monomers would be counted as polymer molecules, since there is no creation of repeating units according to this approach. However, they must be also considered as containing special reactive groups.

Each family of molecules $\mathbf{P}(\mathbf{a}, \mathbf{n})$ is likely not to have a unique molecular weight, because of the dispersion of molecular weights of some of the monomer classes. Let $\mathbf{P}_{\mathrm{j}}$ be a molecule with molecular weight $M\left(P_{j}\right), j$ being a variable used for enumerating the set of all possible molecules. The molecular weight is often not simply the sum of the molecular weights of the monomers that formed $\mathbf{P}_{\mathrm{j}}$, because end-groups are likely to give rise to by-products when they react with other end-groups or other molecules in the reaction medium. So, let $M_{i}$ be the decrease of molecular weight of a polymer molecule when one of its endgroups $\mathbf{A}_{\mathbf{i}}$ reacts. This molecular weight loss can always be supposed to be independent of the nature of this latter reaction. This is rather evident for endgroups like $\mathrm{COCl}$, which loses a $\mathrm{Cl}$ atom when reacting with any group carrying an active hydrogen (-OH, $-\mathrm{NH}_{2}$ and so on), so that it can safely be assumed that $M_{\mathrm{COCl}}=M_{\mathrm{Cl}}=35.5$. In some special cases, like $-\mathrm{CH}_{2} \mathrm{OH}$ in phenol-formaldehyde resins, which may form either methylene $-\mathrm{CH}_{2}$ - with loss of formaldehyde or oxydimethylene bridges $-\mathrm{CH}_{2} \mathrm{OCH}_{2}$-with loss of water when they react, the whole molecular weight of the $-\mathrm{CH}_{2} \mathrm{OH}$ group must be considered lost when it reacts, so that $M_{\mathrm{CH}_{2} \mathrm{OH}}=33$, and $-\mathrm{CH}_{2}-,-\mathrm{CH}_{2} \mathrm{OCH}_{2}-$ must be considered end-groups with $M_{\mathrm{CH}_{2} \mathrm{OCH}_{2}}=44$, $M_{\mathrm{CH}_{1}}=14$, in spite of them being unable to react further (unless their formation becomes reversible).

Mole fraction $\Omega^{m j}$ of subspecies $\mathrm{U}^{\mathrm{mi}}$ is also the probability of choosing a subspecies $U^{m j}$ when randomly picking up a unit $\mathrm{U}^{\mathrm{m}}$, because of the chosen normalization. Since the build-up of polymer species is independent of the nature of the subspecies, if a polymer molecule has $n^{m}$ repeating units $U^{m}$, the probability of having $n^{m 0}, n^{m 1}, \ldots$, numbers of subspecies $\mathrm{U}^{\mathrm{m} 0}, \mathrm{U}^{\mathrm{ml}}, \ldots$, is given by the multinomial distribution with $n^{m}=n^{m 0}+n^{m 1}+\cdots$ and a set of probabilities $\Omega^{m 0}, \Omega^{m 1}, \ldots$ It is shown in Appendix A that the discrete transform of the number molecular weight distribution (NMWD) is related to the discrete transform of VNMND through

$$
\begin{aligned}
\bar{P}(\mu) & =\sum_{j=0}^{\infty} \mu^{M\left(P_{j}\right)} P_{j} \\
& =\widetilde{P}\left[\mu^{M_{1}}, \ldots, \mu^{M_{G}}, \bar{\Omega}^{1}(\mu), \ldots, \bar{\Omega}^{N}(\mu)\right] .
\end{aligned}
$$

Expressions for the NMWD of repeating units in the important cases often encountered in practice of Schulz-Flory and Poisson distributions are given in Table 1. Also, the expressions that result for the discrete transform of the NMWD and the leading aver- 
Table 1. Molecular weight distributions and average molecular weights of classes of repeating units following Schulz-Flory and Poisson's distributions

\begin{tabular}{l}
\hline \multicolumn{1}{c}{ Schulz-Flory distribution } \\
\hline $\bar{\Omega}(\mu)=\frac{(1-p) \mu^{M_{2}}}{1-p \mu^{M_{n}}}$ \\
$(1-p) \bar{M}_{n}=p\left(M_{R}-M_{E}\right)+M_{E}$ \\
$(1-p)^{2} \bar{M}_{w} \bar{M}_{n}=\left(M_{R}-M_{E}\right)^{2} p^{2}+\left(M_{R}^{2}+2 M_{R} M_{E}-2 M_{E}^{2}\right) p+M_{E}^{2}$ \\
$(1-p)^{3} \bar{M}_{z} \bar{M}_{w} \bar{M}_{n}=\left(M_{R}-M_{E}\right)^{3} p^{3}+\left(4 M_{R}^{3}-6 M_{R} M_{E}^{2}+3 M_{E}^{3}\right) p^{2}+\left[\left(M_{R}+M_{E}\right)^{3}-4 M_{E}^{3}\right] p+M_{E}$ \\
$\bar{\Omega}(\mu)=\mu^{M_{\varepsilon}} \exp \left[J\left(\mu^{M_{k}}-1\right)\right] \quad$ Poisson's distribution \\
$\bar{M}_{n}=J M_{R}+M_{E}$ \\
$\bar{M}_{w} \bar{M}_{n}=J M_{R}^{2}+\left(J M_{R}+M_{E}\right)^{2}$ \\
$\bar{M}_{z} \bar{M}_{w} \bar{M}_{n}=J M_{R}^{3}+3 J M_{R}^{2}\left(J M_{R}+M_{E}\right)+\left(J M_{R}+M_{E}\right)^{3}$ \\
\hline
\end{tabular}

Table 2. Relations between leading moments of MWD and of VNMND

$$
\begin{aligned}
\lambda_{M_{1}}= & \sum_{m=1}^{N} \bar{M}_{n}^{m} \lambda^{m}+\sum_{i=1}^{G} M_{i} \lambda_{i} \\
\lambda_{M_{2}}= & \sum_{m=1}^{N} \sum_{n=1}^{N} \bar{M}_{n}^{m} \bar{M}_{n}^{n} \lambda^{m n}+2 \sum_{m=1}^{N} \sum_{i=1}^{G} \bar{M}_{n}^{m} M_{i} \lambda_{i}^{m}+\sum_{i=1}^{G} \sum_{j=1}^{G} M_{i} M_{j} \lambda_{i j}+\sum_{m=1}^{N} \bar{M}_{n}^{m}\left(\bar{M}_{w}^{m}-\bar{M}_{n}^{m}\right) \lambda^{m} \\
\lambda_{M_{2}}= & \sum_{m=1}^{N} \sum_{n=1}^{N} \sum_{p=1}^{N} \bar{M}_{n}^{m} \bar{M}_{n}^{n} \bar{M}_{n}^{p} \lambda^{m n p}+3 \sum_{m=1}^{N} \sum_{n=1}^{N} \sum_{i=1}^{G} \bar{M}_{n}^{m} \bar{M}_{n}^{n} M_{i} \lambda_{i}^{m n}+3 \sum_{m=1}^{N} \sum_{i=1}^{G} \sum_{j=1}^{G} \bar{M}_{n}^{m} M_{i} M_{j} \lambda_{i j}^{m} \\
& +\sum_{i=1}^{G} \sum_{j=1}^{G} \sum_{k=1}^{G} M_{i} M_{j} M_{k} \lambda_{i j k}+3 \sum_{m=1}^{N} \bar{M}_{n}^{m}\left(\bar{M}_{w}^{m}-\bar{M}_{n}^{m}\right)\left(\sum_{n=1}^{N} \bar{M}_{n} \lambda^{m n}+\sum_{i=1}^{G} M_{i} \lambda_{i}^{m}\right) \\
& +\sum_{m=1}^{N}\left[\bar{M}_{z}^{m} \bar{M}_{w}^{m} \bar{M}_{n}^{m}-3 \bar{M}_{w}^{m}\left(\bar{M}_{n}^{m}\right)^{2}+2\left(\bar{M}_{n}^{m}\right)^{3}\right] \lambda^{m}
\end{aligned}
$$

age molecular weights are given for repeating units with Schulz-Flory and Poisson's distributions of their sub-classes, assuming also that their possible molecular weights are evenly distributed according to

$$
M^{j}=j M_{R}+M_{E} \quad(j=0, \infty) .
$$

Integer-order moments of NMWD, $\lambda_{M_{t}}$, allow average molecular weights, such as number-, weight- and $z$-average molecular weights to be obtained as

$$
\begin{aligned}
& \bar{M}_{n}=\lambda_{M_{1}} / \lambda_{M_{0}} \\
& \bar{M}_{w}=\lambda_{M_{2}} / \lambda_{M_{1}} \\
& \bar{M}_{z}=\lambda_{M_{3}} / \lambda_{M_{2}}
\end{aligned}
$$

and these can be obtained by differentiating eq. (2) with respect to $\log \mu$ and setting $\mu=1$. The resulting expressions are given in Table 2. Evaluation of the moments of NMWD requires the set of the moments of the VNMND, which are derivatives of $\bar{P}$ on $\sigma=1_{G}$, $\zeta=1^{N}$ :

$\lambda_{i j k \ldots}^{m n p \ldots}=\Lambda_{i j k \ldots}^{m n p \ldots}\left(\mathbf{1}_{G}, \mathbf{1}^{N}\right)=\frac{\partial \partial \partial \ldots \partial \partial \partial \ldots \vec{P}}{\partial \log \sigma_{i} \partial \log \sigma_{j} \partial \log \sigma_{k} \ldots \partial \log \zeta^{m} \partial \log \zeta^{n} \partial \log \zeta^{p} \ldots}\left(\mathbf{1}_{G}, \mathbf{1}^{N}\right)$. $\lambda_{j}$ and $\lambda^{j}$ are, respectively, the concentrations of endgroups $\mathbf{A}_{j}$ and repeating units $U^{j}$ belonging to finite molecules (the sol), and so the following equalities hold:

$$
A_{j s}=\lambda_{j}=\Lambda_{j}\left(1_{G}, 1^{N}\right)
$$

$$
U_{s}^{j}=\lambda^{j}=\Lambda^{j}\left(\mathbf{1}_{G}, \mathbf{1}^{N}\right) .
$$

The weight fraction of $\mathrm{sol} w_{s}$ is therefore given by

$$
w_{s}=\lambda_{M_{i}}=\sum_{m=1}^{N} \bar{M}_{n}^{m} \lambda^{m}+\sum_{i=1}^{G} M_{j} \lambda_{i}
$$

When dealing with polyadditions, the contributions of the smallest molecules should be subtracted from the moments, as there is always an appreciable amount of monomers along with high molecular weight polymer.

Molecular growth in polycondensation processes occurs through linking of repeating units by the reaction of the end-groups that are attached to them. Intramolecular loops are produced by reaction of two end-groups separated by a finite number of repeating units. Propagation and termination by combination play an analogous rule in polyadditions.

When an end-group $A_{i}$ attached to some repeating

unit $\mathbf{X}$ reacts with another end-group $\mathbf{A}_{\mathbf{j}}$ attached to some repeating unit $\mathrm{Y}$ in another molecule creating a link $L_{i j}$ between the two repeating units, the vector of numbers of end-groups attached to $\mathbf{X}$ changes by 
$v_{i j}=\left[v_{i j 1}, \ldots, v_{i j G}\right]$ and likewise the vector of numbers of end-groups attached to $\mathrm{Y}$ changes by $v_{j i}=$ $\left[v_{j i 1}, \ldots, v_{j i G}\right]$. This is the key concept allowing the analysis of arbitrarily complex polymerizations.

The curing of a tetraepoxide with a diamine will be used as a case study throughout this paper. There are $N=2$ repeating units: the one carrying the two primary amine groups $\left(\mathrm{U}^{1}\right)$ and the other repeating unit carrying the 4 epoxide groups $\left(\mathbf{U}^{2}\right)$. Each primary amine group $\left(A_{1}\right)$ disappears by reaction with one epoxy group $\left(A_{2}\right)$, being transformed into a secondary amine group $\left(\mathbf{A}_{3}\right)$ attached to the same $U^{1}$ repeating unit and making a secondary hydroxyl group $\left(\mathbf{A}_{4}\right)$ appear in the other repeating unit $\left(\mathbf{U}^{2}\right)$ :

$$
\mathbf{X A}_{1}+\mathbf{Y A}_{2} \stackrel{k_{12}}{\longrightarrow} \mathbf{A}_{3} \mathbf{X L}_{12} \mathbf{Y A}_{4} \text {. }
$$

Therefore, $v_{121}=-1, v_{212}=-1, v_{123}=1, v_{214}=1$. The other components of $v_{12}$ and $v_{21}$ are nil, except the ones for the linking groups $\mathbf{L}_{12}$, but it is seldom needed to keep a record of the linking groups.

It is useful to define overall stoichiometric coefficients $\omega_{i j}$, which form a symmetrical matrix:

$$
\omega_{i j}=\omega_{j i}=v_{i j}+v_{j i}
$$

An apparent bimolecular rate constant of the reaction between end-groups $\mathbf{A}_{\mathbf{i}}$ and $\mathbf{A}_{\mathbf{j}}$, named $k_{i j}$, will be defined as the rate of formation of $\mathbf{L}_{\mathrm{ij}}$ divided by the concentrations of $\mathbf{A}_{\mathbf{i}}$ and $\mathbf{A}_{\mathbf{j}}$. This set of rate constants is obviously symmetrical. $k_{i j}$ depends most often on the concentration of end groups and catalysts. For instance, in the case of the reaction between amines and epoxy groups, there is an autocatalysis by the hydroxyl groups (Dusek, 1986) and so

$$
k_{12}=k_{21}=k_{12}^{0}+k_{12}^{\prime} A_{4} .
$$

The complete kinetic scheme of this example is presented in Table 3.

It is possible that parallel reactions exist between the same pair of end-groups $\mathbf{A}_{\mathbf{i}}$ and $\mathbf{A}_{\mathbf{j}}$. A notorious example is the termination reaction between two radicals, which can go through a combination or a dismutation process. In such a case, two (or even more) apparent rate constants $k_{i j}^{\prime}$ and $k_{i j}^{\mathrm{tI}}$, each one with its set of stoichiometric coefficients $v_{i j k}^{1}$ and $v_{i j k}^{\mathrm{II}}$, would have to be introduced.

Reactions of end-groups not leading to intermolecular links, such as intramolecular cyclization, can be modelled using a similar mathematical formalism. The numbers of end-groups of the repeating unit to which they are attached change by a vector $v_{i}^{*}=\left[v_{i 1}^{*}, \ldots, v_{i G}^{*}\right]$, at a rate of reaction given by $k_{i}^{*} A_{i}$. The apparent kinetic constants $k_{i}^{*}$ may also be a function of the concentrations of other end-groups, such as in the example dealing with radical polymerization discussed next.

All pairs of end-groups able to react intramolecularly must be considered pseudo end-groups. The correct definition of end-groups and determination of stoichiometric coefficients is a complex task when intramolecular cyclization reactions need be considered. These reactions were neglected in the treatment of curing an epoxy resin as dealt with in this work.

Radical copolymerization of $N$ polyvinyl compounds $\mathrm{X}^{n}\left(=\mathrm{CH}_{2}\right)_{f^{n}}$ or $\mathbf{U}^{n}\left(\mathbf{A}_{n}\right)_{f^{n}}(n=1, N)$, with penultimate effects in propagation, has a great practical importance and is a good example of system with reactions involving end-groups without forming links, such as termination by dismutation and transfer reactions. Its mechanism and corresponding set of

Table 3. Kinetic scheme of the reaction between a tetraepoxide and a diamine

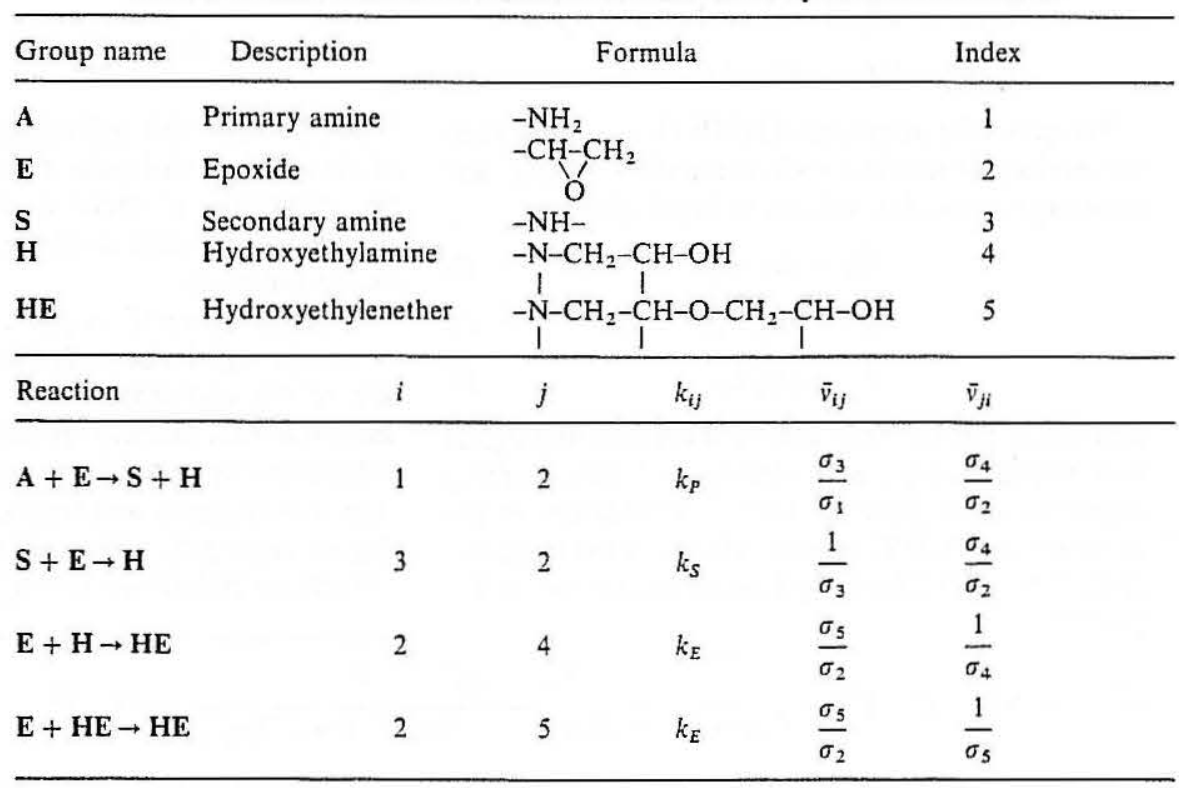

Parameters used in simulation: $k_{S} / k_{P}=0.2$ and $k_{E} / k_{P}=0.055$.

Molecular weights of monomers: $M^{1}=248$ and $M^{2}=422$; for all end-groups $M_{i}=0$. 
stoichiometric coefficients is summarized in Appen$\operatorname{dix} \mathrm{B}$. Not all reactions in radical polymerization can be analysed with this approach, because of the assumption of reaction irreversibility. For instance, depropagation and $\beta$-scission of radicals cannot be taken into account. From this point of view, Villermaux and Blavier's tendency model (Villermaux and Blavier, 1984) remains more general than our proposed model, although it suffers from the drawback of not being able to cope with strongly nonli. ar polymerizations.

One should not overlook the possibility of a breakdown of the validity of the principle of the equal reactivity beyond gel point. Some experimental evidence (Argyropoulos et al., 1987b) points to diffusional limitations having a different effect on small and large polymer molecules, these latter being entrapped in gel. No quantitative model for these effects was put forward up to now, which is not surprising, in view of the difficulty of gathering the necessary experimental data. However, the overall picture of post-gel polymerization according to classical theory (Flory, 1941a-c, 1942, 1947; Stockmayer, 1943, 1944) is fairly accurate, with the exception of the above-mentioned effect (Argyropoulos and Bolker, 1986; Argyropoulos et al., 1987a, c). It seems justified to develop a mathematical treatment of non-linear polymerizations using the principle of the equal reactivity, keeping in mind its main value as a first approximation of their behaviour after gelation.

\section{Rate equations}

Using the principle of the equal reactivity and the above definitions of rate constants and stoichiometric coefficients, the rate equation for the end-groups can be written as follows:

$$
R_{\mathrm{A}_{\mathrm{k}}}=\sum_{i=1}^{G} \sum_{j=1}^{G} k_{i j} v_{i j k} A_{i} A_{j}+\sum_{i=1}^{G} k_{i}^{*} v_{i k}^{*} A_{i} .
$$

So, according to the previous definitions, each pair of end-groups $\mathbf{A}_{\mathbf{i}}$ and $\mathbf{A}_{\mathbf{j}}$ in molecules $\mathbf{P}(\mathbf{a}, \mathbf{m})$ and $\mathbf{P}(\mathbf{b}, \mathbf{n})$ reacts with rate $a_{i} b_{j} k_{i j} P(\mathbf{a}, \mathbf{m}) P(\mathbf{b}, \mathbf{n})$ forming a link between repeating units and thus yields a molecule $\mathbf{P}\left(\mathbf{a}+\mathbf{b}+\omega_{i j}, \mathbf{m}+\mathbf{n}\right):$

$$
\mathbf{P}(\mathbf{a}, \mathbf{m})+\mathbf{P}(\mathbf{b}, \mathbf{n}) \stackrel{a_{i} b_{j} k_{i j}}{\longrightarrow} \mathbf{P}\left(\mathbf{a}+\mathbf{b}+\omega_{i j}, \mathbf{m}+\mathbf{n}\right) .
$$

A reaction of its end-groups $\mathbf{A}_{\mathbf{i}}$ not forming intermolecular links converts molecule $\mathbf{P}(\mathbf{a}, \mathbf{n})$ into a molecule $\mathbf{P}\left(\mathbf{a}+v_{i}^{*}, \mathbf{n}\right)$ :

$$
\mathbf{P}(\mathbf{a}, \mathbf{n}) \stackrel{a_{k} k_{i}^{*}}{\longrightarrow} \mathbf{P}\left(\mathbf{a}+v_{i}^{*}, \mathbf{n}\right)
$$

The rate of formation of polymer species $\mathbf{P}(\mathbf{a}, \mathbf{n})$ is therefore

$$
\begin{aligned}
R_{\mathbf{P}(\mathrm{a}, \mathrm{n})}= & \frac{1}{2} \sum_{i=1}^{G} \sum_{j=1}^{G} k_{i j}\left[\sum_{b_{1}=0}^{b_{1}-\omega_{i j t}} \cdots \sum_{b_{G}=0}^{b_{G}-\omega_{j, G}} \sum_{m^{1}=0}^{n^{1}}\right. \\
& \ldots \sum_{m^{N}=0}^{n^{N}} b_{i} P\left(b_{1}, \ldots, b_{G}, m^{1}, \ldots, m^{N}\right)
\end{aligned}
$$

$$
\begin{aligned}
& \times\left(a_{j}-b_{j}-\omega_{i j j}\right) P\left(a_{1}-b_{1}-\omega_{i j 1}, \ldots, a_{G}\right. \\
& \left.-b_{G}-\omega_{i j G}, n^{1}-m^{1}, \ldots, n^{N}-m^{N}\right) \\
& \left.-a_{i} P\left(a_{1}, \ldots, a_{G}, n^{1}, \ldots, n^{N}\right) A_{j}\right] \\
& +\sum_{i=1}^{G} k_{i}^{*} \sum_{m^{1}=0}^{n^{1}} \ldots \sum_{m^{N}=0}^{n^{N}}\left[\left(a_{i}-v_{i i}^{*}\right)\right. \\
& \times P\left(a_{1}-v_{i 1}^{*}, \ldots, a_{G}-v_{i G}^{*}, n^{1}, \ldots, n^{N}\right) \\
& \left.-a_{i} P\left(a_{1}, \ldots, a_{G}, n^{1}, \ldots, n^{N}\right)\right] .
\end{aligned}
$$

The rate of formation of species $P\left(a_{1}, \ldots, \mathbf{a}_{G}\right.$, $n^{1}, \ldots, n^{N}$ ) depends only on the concentrations of species with lower numbers of end-groups and of repeating units. After insertion of eq. (13) in a mass balance equation of polymer species, a closed system of equations results, from which any set of $P\left(a_{1}, \ldots, a_{G}, n^{1}, \ldots, n^{N}\right)$ with $0 \leqslant a_{i} \leqslant a_{i, \mathrm{MAx}}$, $0 \leqslant n^{m} \leqslant n_{\text {MAX }}^{\mathrm{m}}, 1 \leqslant i \leqslant G, 1 \leqslant m \leqslant N$ can be computed. However, because of the multiple embedded sums, the evaluation of ' requires $O\left(a_{1, \operatorname{MAx}} \ldots a_{G, \operatorname{MAx}} n_{\mathrm{MAX}}^{1} \ldots n_{\mathrm{MAX}}^{N}\right)^{2}$ operations. This is likely to be a huge number, unless the concentrations of the first oligomers are the only one to be evaluated.

Fortunately, the approach suggested by Costa and Villermaux (1988) or Mills $(1986 \mathrm{a}-\mathrm{c})$, based on the evaluation and numerical inversion of discrete transforms also works for this kind of problems and will be used next. Its first step consists in obtaining the discrete transform of rate law [eq. (13)]:

$$
\begin{gathered}
\tilde{R}_{\mathrm{P}}(\sigma, \zeta)=\sum_{i=1}^{G} \sum_{j=1}^{G} k_{i j} \frac{\partial \bar{P}}{\partial \log \sigma_{i}}\left(\frac{1}{2} \bar{\omega}_{i j} \frac{\partial \bar{P}}{\partial \log \sigma_{j}}-A_{j}\right) \\
+\sum_{i=1}^{G} k_{i}^{*} \frac{\partial \bar{P}}{\partial \log \sigma_{i}}\left(\bar{v}_{i}^{*}-1\right) \\
\bar{\omega}_{i j}(\sigma)=\prod_{k=1}^{G} \sigma_{k}^{\left(\omega_{i j k}\right.}=\bar{v}_{i j} \bar{v}_{j i} \\
\bar{v}_{i}^{*}(\sigma)=\prod_{k=1}^{G} \sigma_{k}^{v_{i}^{*}} .
\end{gathered}
$$

\section{EVALUATION OF MWD AND AVERAGE MOLECULAR WEIGHTS IN IDEAL REACTORS}

Integration of mass balance equation of polymer species Prediction of polymer properties will now be carried out for ideal reactors. A non-steady state perfectly mixed continuous stirred tank reactor (CSTR) is a convenient starting point, since batch/plug flow and semi-batch reactors can be considered as special cases.

The overall mass balance should be first considered. Reaction of one mole of end-groups $A_{i}$ and one mole of $\mathrm{A}_{\mathrm{j}}$ is supposed to change volume by $W_{i j}$ and the reaction of end-group $\mathbf{A}_{\mathbf{i}}$ without forming a linking group is supposed to change the volume by $W_{i}^{*}$. A relative change of reactor volume by chemical reaction $R_{V}$ is found by adding all these contributions. If mixing volume is neglected, inlet and outlet volume 
flow rates and reaction volume are related by

$$
\begin{aligned}
\frac{\mathrm{d} \log V}{\mathrm{~d} t}= & \frac{1}{2} \sum_{i=1}^{G} \sum_{j=1}^{G} k_{i j} A_{i} A_{j} W_{i j} \\
& +\sum_{i=1}^{G} k_{i}^{*} A_{i} W_{i}^{*}+\frac{Q_{F}(t)-Q(t)}{V} \\
= & R_{V}+\frac{Q_{F}(t)-Q(t)}{V} .
\end{aligned}
$$

This balance also holds in a batch reactor (where $Q_{F}=Q=0$ ) and in a semi-batch reactor (where only $Q=0$ ). It is natural to consider that $V$ is constant in a CSTR and let eq. (17) be used for relating inlet and outlet flow rates. In a semi-batch reactor, it is used for computing reaction volume $V(t)$.

Therefore, a generic molecular species, such as some end-group $A_{k}$, verifies the following mass balance equation with space time $\tau$ defined as the ratio of reaction volume by inlet flow rate:

$$
\begin{aligned}
\frac{\partial A_{k}}{\partial t} & =R_{\mathrm{A}_{\mathrm{k}}}+\frac{A_{k F}(t)-A_{k}}{\tau(t)}-R_{V} A_{k} \\
\left.A_{k}\right|_{t=0} & =A_{k 0} .
\end{aligned}
$$

Discrete transforms of number molecular number distributions or moments of these also verify this generic mass balance. So, the NMND of polymer species can be found by integrating the non-linear first-order partial differential equation

$$
\begin{aligned}
\frac{\partial \bar{P}}{\partial t}= & \sum_{i=1}^{G} \sum_{j=1}^{G} k_{i j} \frac{\partial \bar{P}}{\partial \log \sigma_{i}}\left(\frac{1}{2} \bar{\omega}_{i j} \frac{\partial \bar{P}}{\partial \log \sigma_{j}}-A_{j}\right) \\
& +\sum_{i=1}^{G} k_{i}^{*} \frac{\partial \bar{P}}{\partial \log \sigma_{i}}\left(\bar{v}_{i}^{*}-1\right) \\
& +\frac{\bar{P}_{F}(t)-\bar{P}}{\tau}-R_{V} \bar{P} \\
\left.\bar{P}\right|_{t=0}= & \bar{P}_{0}(\sigma, \zeta) .
\end{aligned}
$$

Because of the way the time variable $t$ appears in eq. (20),

$$
\begin{aligned}
\frac{\partial \bar{P}}{\partial t} & =F\left(t, \sigma_{1}, \ldots, \sigma_{G}, \bar{P}, \frac{\partial \bar{P}}{\partial \log \sigma_{1}}, \ldots, \frac{\partial \bar{P}}{\partial \log \sigma_{G}}\right) \\
& =F\left(t, \sigma_{1}, \ldots, \sigma_{G}, \bar{P}, \Lambda_{1}, \ldots, \Lambda_{G}\right)
\end{aligned}
$$

a special adaptation of the method of characteristics (Courant and Hilbert, 1962) is worth doing, as will be shown next. Partial differentiation with respect to $\log \sigma_{1}, \ldots, \log \sigma_{G}$ leads to a system now including $G$ new partial differential equations for the $\Lambda_{1}, \ldots, \Lambda_{G}$ :

$$
\frac{\partial \Lambda_{k}}{\partial t}-\sum_{i=1}^{G} \frac{\partial F}{\partial \Lambda_{i}} \frac{\partial \Lambda_{k}}{\partial \log \sigma_{i}}=\frac{\partial F}{\partial \log \sigma_{k}}+\frac{\partial F}{\partial \bar{P}} \Lambda_{k} \quad(k=1, G)
$$

(notice the use of the identities $\partial \Lambda_{i} / \partial \log \sigma_{k}=$ $\partial^{2} \bar{P} / \partial \log \sigma_{i} \partial \log \sigma_{k}=\partial \Lambda_{k} / \partial \log \sigma_{i}$ ). These new partial differential equations are semi-linear, and along the characteristic curves defined by

$$
\frac{\mathrm{d} \log \sigma_{k}}{\mathrm{~d} t}=-\frac{\partial F}{\partial \Lambda_{k}}
$$

$\Lambda_{1}, \ldots, \Lambda_{G}$ verify

$$
\frac{\mathrm{d} \Lambda_{k}}{\mathrm{~d} t}=\frac{\partial F}{\partial \log \sigma_{k}}+\frac{\partial F}{\partial \bar{P}} \Lambda_{k} .
$$

Also, along the characteristics:

$$
\begin{aligned}
\frac{\mathrm{d} \bar{P}}{\mathrm{~d} t} & =\sum_{i=1}^{G} \frac{\partial \bar{P}}{\partial \log \sigma_{i}} \frac{\mathrm{d} \log \sigma_{i}}{\mathrm{~d} t}=\sum_{i=1}^{G} \Lambda_{i} \frac{\mathrm{d} \log \sigma_{i}}{\mathrm{~d} t} \\
& =-\sum_{i=1}^{G} \Lambda_{i} \frac{\partial F}{\partial \Lambda_{i}}
\end{aligned}
$$

Carrying out the substitution in eqs (24)-(26) of the required partial derivatives of the right-hand side of eq. (20), $\partial F / \partial \log \sigma_{i}, \partial F / \partial \Lambda_{i}$ and $\partial F / \partial \bar{P}$, the following system of $2 G+1$ ordinary differential equations along the characteristics $\sigma(t)$ starting at $\sigma=\sigma_{0}(\zeta$ is constant along the characteristics) is obtained:

$$
\begin{gathered}
\frac{\mathrm{d} \bar{P}}{\mathrm{~d} t}=-\frac{1}{2} \sum_{i=1}^{G} \sum_{j=1}^{G} k_{i j} \bar{\omega}_{i j} \Lambda_{i} \Lambda_{j} \\
+\frac{\bar{P}_{F}(t)-\bar{P}}{\tau}-R_{V} \bar{P} \\
\frac{\mathrm{d} \log \sigma_{k}}{\mathrm{~d} t}=\sum_{i=1}^{G} k_{k i}\left(A_{i}-\bar{\omega}_{k i} \Lambda_{i}\right)+k_{k}^{*}\left(1-\bar{v}_{k}^{*}\right) \\
\frac{\mathrm{d} \Lambda_{k}}{\mathrm{~d} t}=\frac{1}{2} \sum_{i=1}^{G} \sum_{j=1}^{G} k_{i j} \omega_{i j k} \bar{\omega}_{i j} \Lambda_{i} \Lambda_{j}+\sum_{i=1}^{G} k_{i}^{*} v_{i k}^{*} \bar{v}_{i}^{*} \Lambda_{i} \\
+\frac{\Lambda_{k F}(t)-\Lambda_{k}}{\tau}-R_{Y} \Lambda_{k} \\
\bar{P}_{\left.\right|_{t}=0}=\bar{P}_{0}\left(\sigma_{0}, \zeta\right) \\
\left.\sigma_{k}\right|_{t=0}=\sigma_{k 0} \\
\left.\Lambda_{k}\right|_{t=0}=\Lambda_{k 0}\left(\sigma_{0}, \zeta\right)=\frac{\partial \bar{P}_{0}}{\partial \log \sigma_{k 0}}\left(\sigma_{0}, \zeta\right)
\end{gathered}
$$

In order that a certain characteristic passes through a previously specified value of $\sigma$ at a time $t$, a system of $G$ algebraic equations must be solved to find the starting vector $\sigma_{0}$ at time $t=0$ :

$$
\log \sigma_{k}\left(\log \sigma_{0}, \zeta\right)-\log \sigma_{k}=0 .
$$

The Newton-Raphson method can be used to solve this system of algebraic equations, with some care in the choice of the starting values of $\sigma_{0}$. The Jacobian of system (33),

$$
S_{k 1}=\frac{\partial \log \sigma_{k}}{\partial \log \sigma_{l 0}}
$$

can be found after introducing the auxiliary sensitivities

$$
S_{i}^{k}=\frac{\partial \log \Lambda_{k}}{\partial \log \sigma_{l 0}}
$$

and solving eqs (28)-(29) and eqs (31)-(32) simultaneously with the additional $2 G^{2}$ ordinary differential 
equations:

$$
\begin{aligned}
\frac{\mathrm{d} S_{k l}}{\mathrm{~d} t}= & -\sum_{i=1}^{G} k_{k i} \bar{\omega}_{k i}\left(S_{l}^{i}+\Lambda_{i} \sum_{j=1}^{G} \omega_{k i j} S_{j l}\right) \\
& -k_{k}^{*} \bar{v}_{k}^{*} \sum_{j=1}^{G} v_{k j}^{*} S_{j l} \\
\frac{\mathrm{d} S_{l}^{k}}{\mathrm{~d} t}= & \sum_{i=1}^{G} \sum_{j=1}^{G} k_{i j} \omega_{i j k} \bar{\omega}_{i j} \Lambda_{i}\left(S_{l}^{j}+\frac{1}{2} \Lambda_{j} \sum_{h=1}^{G} \omega_{i j h} S_{h l}\right) \\
& +\sum_{i=1}^{G} k_{i}^{*} v_{i k}^{*} \bar{v}_{i}^{*}\left(S_{l}^{i}+\Lambda_{i} \sum_{j=1}^{G} v_{i j}^{*} S_{j l}\right) \\
& +\frac{\Lambda_{k i F}(t)-S_{l}^{k}}{\tau}-R_{V} S_{l}^{k} \\
\left.S_{k l}\right|_{t=0}= & \begin{cases}1 & \text { if } k=l \\
0 & \text { otherwise }\end{cases} \\
\left.S_{l}^{k}\right|_{t=0}= & \Lambda_{k l 0}=\frac{\partial^{2} \bar{P}_{0}}{\partial \log \sigma_{k 0} \partial \log \sigma_{l 0}}\left(\sigma_{0}, \zeta\right) .
\end{aligned}
$$

Most often, the marginal distribution with respect to the number of repeating units $\bar{P}\left(\mathbf{1}_{G}, \zeta\right)$ is sought and so $\sigma=1_{G}$. If also $\zeta=1^{N}$, the starting point $\sigma_{0}$ should be a vector of numbers as close to zero as necessary, so that $\sigma=\mathbf{1}_{G}$ is approached only by values of $\sigma_{i}<1$. Now, a possible solution of eqs (27)-(32) for $\sigma=1_{G}$ and $\zeta=1^{N}$ leads to $\Lambda_{i}=A_{i}$ and $\sigma_{0}=1_{G}$. If NewtonRaphson's method converges towards this solution, it means that the gelation time has not yet been attained, because all reactive groups belong to finite molecules. If $\sigma=\mathbf{1}_{G}$ can be attained with some or all $\sigma_{i 0}<1$, it will result in $\Lambda_{i}<A_{i}$ for some $i$ and gelation has occurred.

In order to estimate any marginal distribution according to the number of repeating units $\mathrm{U}^{\mathrm{m}}$ by numerical inversion of $\bar{P}, n_{\mathrm{MAx}}^{m}$ values of this discrete transform must be evaluated along a circle in complex plane with $\left|\zeta^{m}\right|=$ constant $\leqslant 1$, with all other values of $\zeta^{n}$ or $\sigma_{i}$ equal to 1 . The starting value of vector $\sigma_{0}$ for the computation of each of these points should be the one found in the previous computation. The first of all should be a vector of small positive real numbers for the evaluation along the real positive axis.

Evaluation of average molecular weights and weight fraction of gel

In order to compute the moments of the VNMND, rate equations for the derivatives of $\bar{P}$ are obtained by differentiation of eq. (14) with respect to $\log \sigma_{k}$ and/or $\log \zeta^{m}:$

$$
\begin{aligned}
R_{\Lambda_{k}}= & \sum_{i=1}^{G} \sum_{j=1}^{G} k_{i j}\left[\frac{\partial \Lambda_{k}}{\partial \log \sigma_{i}}\left(\bar{\omega}_{i j} \Lambda_{j}-A_{j}\right)\right. \\
& \left.+\frac{1}{2} \omega_{i j k} \vec{v}_{i j} \Lambda_{i} \Lambda_{j}\right] \\
& +\sum_{i=1}^{G} k_{i}^{*}\left[\frac{\partial \Lambda_{k}}{\partial \log \sigma_{i}}\left(\bar{v}_{i}^{*}-1\right)+v_{i k}^{*} \bar{v}_{i}^{*} \Lambda_{i}\right] \\
R_{\Lambda^{4}}= & \sum_{i=1}^{G} \sum_{j=1}^{G} k_{i j} \frac{\partial \Lambda^{k}}{\partial \log \sigma_{i}}\left(\bar{\omega}_{i j} \Lambda_{j}-A_{j}\right)
\end{aligned}
$$

$$
\begin{aligned}
& +\sum_{i=1}^{c} k_{i}^{*} \frac{\partial \Lambda^{k}}{\partial \log \sigma_{i}}\left(\vec{v}_{i}^{*}-1\right) \\
& R_{\Lambda_{u}}=\sum_{i=1}^{G} \sum_{j=1}^{G} k_{i j}\left\{\frac{\partial \Lambda_{k l}}{\partial \log \sigma_{i}}\left(\bar{\omega}_{i j} \Lambda_{j}-A_{j}\right)\right. \\
& +\bar{\omega}_{i j}\left[\frac{1}{2} \omega_{i j k} \omega_{i j i} \Lambda_{i} \Lambda_{j}+\Lambda_{i k} \Lambda_{j l}\right. \\
& \left.\left.+\Lambda_{j}\left(\omega_{i j k} \Lambda_{i l}+\omega_{i j l} \Lambda_{i k}\right)\right]\right\} \\
& +\sum_{i=1}^{G} k_{i}^{*}\left[\frac{\partial \Lambda_{k l}}{\partial \log \sigma_{i}}\left(\bar{v}_{i}^{*}-1\right)\right. \\
& \left.\bar{v}_{i}^{*}\left(v_{i k}^{*} \Lambda_{i l}+v_{i l}^{*} \Lambda_{i k}+v_{i k}^{*} v_{i i}^{*} \Lambda_{i}\right)\right] \\
& R_{\Lambda_{i}^{i}}=\sum_{i=1}^{G} \sum_{j=1}^{G} k_{i j}\left[\frac{\partial \Lambda_{l}^{k}}{\partial \log \sigma_{i}}\left(\bar{\omega}_{i j} \Lambda_{j}-A_{j}\right)\right. \\
& \left.+\bar{\omega}_{i j}\left(\Lambda_{i}^{k} \Lambda_{j l}+\omega_{i j l} \Lambda_{j} \Lambda_{i}^{k}\right)\right] \\
& +\sum_{i=1}^{G} k_{i}^{*}\left[\frac{\partial \Lambda_{l}^{k}}{\partial \log \sigma_{i}}\left(\bar{v}_{i}^{*}-1\right)+v_{i l}^{*} v_{i}^{*} \Lambda_{i}^{k}\right] \\
& R_{\Lambda^{\prime \prime}}=\sum_{i=1}^{G} \sum_{j=1}^{G} k_{i j}\left[\frac{\partial \Lambda^{k l}}{\partial \log \sigma_{i}}\left(\bar{\omega}_{i j} \Lambda_{j}-A_{j}\right)+\bar{\omega}_{i j} \Lambda_{i}^{k} \Lambda_{j}^{l}\right] \\
& +\sum_{i=1}^{G} k_{i}^{*} \frac{\partial \Lambda^{k l}}{\partial \log \sigma_{i}}\left(\bar{v}_{i}^{*}-1\right) \\
& R_{\Lambda_{\mathrm{slm}}}=\sum_{i=1}^{G} \sum_{j=1}^{G} k_{i j}\left\{\frac{\partial \Lambda_{k l m}}{\partial \log \sigma_{i}}\left(\bar{\omega}_{i j} \Lambda_{j}-A_{j}\right)\right. \\
& +\bar{\omega}_{i j}\left[\Lambda_{i k l} \Lambda_{j m}+\Lambda_{i k m} \Lambda_{j l}+\Lambda_{i l m} \Lambda_{j k}\right. \\
& +\omega_{i j k} \Lambda_{i l} \Lambda_{j m}+\omega_{i j l} \Lambda_{i k} \Lambda_{j m}+\omega_{i j m} \Lambda_{i k} \Lambda_{j l} \\
& +\Lambda_{j}\left(\frac{1}{2} \omega_{i j k} \omega_{i j l} \omega_{i j m} \Lambda_{i}+\omega_{i j k} \Lambda_{i l m}\right. \\
& +\omega_{i j l} \Lambda_{i k m}+\omega_{i j m} \Lambda_{i k l}+\omega_{i j k} \omega_{i j l} \Lambda_{i m} \\
& \left.\left.\left.+\omega_{i j k} \omega_{i j m} \Lambda_{i l}+\omega_{i j l} \omega_{i j m} \Lambda_{i k}\right)\right]\right\} \\
& +\sum_{i=1}^{G} k_{i}^{*}\left[\frac{\partial \Lambda_{k l m}}{\partial \log \sigma_{i}}\left(\bar{v}_{i}^{*}-1\right)+\bar{v}_{i}^{*}\left(v_{i k}^{*} \Lambda_{i l m}\right.\right. \\
& +v_{i l}^{*} \Lambda_{i k m}+v_{i m}^{*} \Lambda_{i k l}+v_{i k}^{*} v_{i l}^{*} \Lambda_{i m}+v_{i k}^{*} v_{i m}^{*} \Lambda_{i l} \\
& \left.\left.+v_{i i}^{*} v_{i m}^{*} \Lambda_{i k}+v_{i k}^{*} v_{i l}^{*} v_{i m}^{*} \Lambda_{i}\right)\right]
\end{aligned}
$$

$$
\begin{aligned}
R_{\Lambda_{i m}}= & \sum_{i=1}^{G} \sum_{j=1}^{G} k_{i j}\left\{\frac{\partial \Lambda_{i m}^{k}}{\partial \log \sigma_{i}}\left(\bar{\omega}_{i j} \Lambda_{j}-A_{j}\right)\right. \\
& +\bar{\omega}_{i j}\left[\Lambda_{i}^{k} \Lambda_{j l m}+\Lambda_{i l}^{k} \Lambda_{j m}+\Lambda_{i m}^{k} \Lambda_{j l}\right. \\
& +\omega_{i j l}\left(\Lambda_{j m} \Lambda_{i}^{k}+\Lambda_{j} \Lambda_{i m}^{k}\right)+\omega_{i j m}\left(\Lambda_{j l} \Lambda_{i}^{k}\right. \\
& \left.\left.\left.+\Lambda_{j} \Lambda_{i l}^{k}\right)+\omega_{i j l} \omega_{i j m} \Lambda_{j} \Lambda_{i}^{k}\right]\right\} \\
& +\sum_{i=1}^{G} k_{i}^{*}\left[\frac{\partial \Lambda_{l m}^{k}}{\partial \log \sigma_{i}}\left(\bar{v}_{i}^{*}-1\right)\right. \\
& \left.+\bar{v}_{i}^{*}\left(v_{i i}^{*} \Lambda_{i m}^{k}+v_{i m}^{*} \Lambda_{i l}^{k}+v_{i l}^{*} v_{i m}^{*} \Lambda_{i}^{k}\right)\right]
\end{aligned}
$$




$$
\begin{aligned}
R_{\Lambda_{m}^{u}}= & \sum_{i=1}^{G} \sum_{j=1}^{G} k_{i j}\left\{\frac{\partial \Lambda_{m}^{k l}}{\partial \log \sigma_{i}}\left(\bar{\omega}_{i j} \Lambda_{j}-A_{j}\right)\right. \\
& +\bar{\omega}_{i j}\left[\Lambda_{i}^{k l} \Lambda_{j m}+\Lambda_{i m}^{k} \Lambda_{j}^{l}+\Lambda_{i}^{k} \Lambda_{j m}^{l}\right. \\
& \left.\left.+\omega_{i j m}\left(\Lambda_{j} \Lambda_{i}^{k l}+\Lambda_{i}^{k} \Lambda_{j}^{l}\right)\right]\right\} \\
& +\sum_{i=1}^{G} k_{i}^{*}\left[\frac{\partial \Lambda_{m}^{k l}}{\partial \log \sigma_{i}}\left(\bar{v}_{i}^{*}-1\right)+v_{i m}^{*} \bar{v}_{i}^{*} \Lambda_{i}^{k l}\right] \\
R_{\Lambda^{k i m}}= & \sum_{i=1}^{G} \sum_{j=1}^{G} k_{i j}\left[\frac{\partial \Lambda^{k i m}}{\partial \log \sigma_{i}}\left(\bar{\omega}_{i j} \Lambda_{j}-A_{j}\right)\right. \\
& \left.+\bar{\omega}_{i j}\left(\Lambda_{i}^{k l} \Lambda_{j}^{m}+\Lambda_{i}^{k m} \Lambda_{j}^{l}+\Lambda_{i}^{k} \Lambda_{j}^{l m}\right)\right] \\
& +\sum_{i=1}^{G} k_{i}^{*} \frac{\partial \Lambda^{k l m}}{\partial \log \sigma_{i}}\left(\bar{v}_{i}^{*}-1\right) .
\end{aligned}
$$

Inserting these expressions in mass balance equations, such as the one in a CSTR, a system of non-linear first-order partial differential equations results. Its characteristics are the same as the ones used for computing $\bar{P}$, eqs (27)-(32). Along with them, the derivatives of $\bar{P}$ verify

$$
\begin{aligned}
& \frac{\mathrm{d} \Lambda^{k}}{\mathrm{~d} t}=\frac{\Lambda_{F}^{k}(t)-\Lambda^{k}}{\tau}-R_{V} \Lambda^{k} \\
& \frac{\mathrm{d} \Lambda_{k l}}{\mathrm{~d} t}=\sum_{i=1}^{G} \sum_{j=1}^{G} k_{i j} \bar{\omega}_{i j}\left[\Lambda_{i k} \Lambda_{j l}+\Lambda_{j}\left(\omega_{i j k} \Lambda_{i l}\right.\right. \\
& \left.\left.+\omega_{i j l} \Lambda_{i k}\right)+\frac{1}{2} \omega_{i j k} \omega_{i j l} \Lambda_{i} \Lambda_{j}\right] \\
& +\sum_{i=1}^{G} k_{i}^{*}\left[\bar{v}_{i}^{*}\left(v_{i k}^{*} \Lambda_{i l}+v_{i l}^{*} \Lambda_{i k}+v_{i k}^{*} v_{i i}^{*} \Lambda_{i}\right)\right] \\
& +\frac{\Lambda_{k l F}(t)-\Lambda_{k l}}{\tau}-R_{V} \Lambda_{k l} \\
& \frac{\mathrm{d} \Lambda_{l}^{k}}{\mathrm{~d} t}=\sum_{i=1}^{G} \sum_{j=1}^{G} k_{i j} \bar{\omega}_{i j}\left(\Lambda_{i}^{k} \Lambda_{j l}+v_{i j l} \Lambda_{j} \Lambda_{i}^{k}\right) \\
& +\sum_{i=1}^{G} k_{i}^{*} v_{i l}^{*} \bar{v}_{i}^{*} \Lambda_{i}^{k}+\frac{\Lambda_{l F}^{k}(t)-\Lambda_{l}^{k}}{\tau}-R_{V} \Lambda_{i}^{k} \\
& \frac{\mathrm{d} \Lambda^{k l}}{\mathrm{~d} t}=\sum_{i=1}^{G} \sum_{j=1}^{G} k_{i j} \bar{\omega}_{i j} \Lambda_{i}^{k} \Lambda_{j}^{t}+\frac{\Lambda_{F}^{k l}(t)-\Lambda^{k l}}{\tau}-R_{V} \Lambda^{k l} \\
& \frac{\mathrm{d} \Lambda_{k l m}}{\mathrm{~d} t}=\sum_{i=1}^{G} \sum_{j=1}^{G} k_{i j} \bar{\omega}_{i j}\left[\Lambda_{i k l} \Lambda_{j m}+\Lambda_{i k m} \Lambda_{j l}+\Lambda_{i l m} \Lambda_{j k}\right. \\
& +\omega_{i j k} \Lambda_{i l} \Lambda_{j m}+\omega_{i j l} \Lambda_{i k} \Lambda_{j m}+\omega_{i j m} \Lambda_{i k} \Lambda_{j l} \\
& +\Lambda_{j}\left(\omega_{i j k} \Lambda_{i l m}+\omega_{i j l} \Lambda_{i k m}+\omega_{i j m} \Lambda_{i k l}\right. \\
& +\omega_{i j k} \omega_{i j l} \Lambda_{i m}+\omega_{i j k} \omega_{i j m} \Lambda_{i l}+\omega_{i j l} \omega_{i j m} \Lambda_{i k} \\
& \left.\left.+\frac{1}{2} \omega_{i j k} \omega_{i j l} \omega_{i j m} \Lambda_{i}\right)\right]+\sum_{i=1}^{G} k_{i}^{*} \bar{v}_{i}^{*}\left(v_{i k}^{*} \Lambda_{i l m}\right. \\
& +v_{i l}^{*} \Lambda_{i k m}+v_{i m}^{*} \Lambda_{i k l}+v_{i k}^{*} v_{i l}^{*} \Lambda_{i m} \\
& \left.+v_{i k}^{*} v_{i m}^{*} \Lambda_{i l}+v_{i l}^{*} v_{i m}^{*} \Lambda_{i k}+v_{i k}^{*} v_{i l}^{*} v_{i m}^{*} \Lambda_{i}\right) \\
& +\frac{\Lambda_{k l m F}(t)-\Lambda_{k l m}}{\tau}-R_{V} \Lambda_{k l m}
\end{aligned}
$$

$$
\begin{aligned}
& \frac{\mathrm{d} \Lambda_{l m}^{k}}{\mathrm{~d} t}=\sum_{i=1}^{G} \sum_{j=1}^{G} k_{i j} \bar{\omega}_{i j}\left[\Lambda_{i}^{k} \Lambda_{j l m}+\Lambda_{i l}^{k} \Lambda_{j m}+\Lambda_{i m}^{k} \Lambda_{j l}\right. \\
& +\omega_{i j l}\left(\Lambda_{j m} \Lambda_{i}^{k}+\Lambda_{j} \Lambda_{i m}^{k}\right) \\
& \left.+\omega_{i j m}\left(\Lambda_{j l} \Lambda_{i}^{k}+\Lambda_{j} \Lambda_{i l}^{k}\right)+\omega_{i j l} \omega_{i j m} \Lambda_{j} \Lambda_{i}^{k}\right] \\
& +\sum_{i=1}^{G} k_{i}^{*} \bar{v}_{i}^{*}\left(v_{i l}^{*} \Lambda_{i m}^{k}+v_{i m}^{*} \Lambda_{i l}^{k}+v_{i l}^{*} v_{i m}^{*} \Lambda_{i}^{k}\right) \\
& +\frac{\Lambda_{l m F}^{k}(t)-\Lambda_{i m}^{k}}{\tau}-R_{V} \Lambda_{l m}^{k} \\
& \frac{\mathrm{d} \Lambda_{m}^{k l}}{\mathrm{~d} t}=\sum_{i=1}^{G} \sum_{j=1}^{G} k_{i j} \bar{\omega}_{i j}\left[\Lambda_{i}^{k l} \Lambda_{j m}+\Lambda_{i m}^{k} \Lambda_{j}^{l}+\Lambda_{i}^{k} \Lambda_{j m}^{l}\right. \\
& \left.+\omega_{i j m}\left(\Lambda_{j} \Lambda_{i}^{k l}+\Lambda_{i}^{k} \Lambda_{j}^{i}\right)\right]+\sum_{i=1}^{G} k_{i}^{*} v_{i m}^{*} \bar{v}_{i}^{*} \Lambda_{i}^{k l} \\
& +\frac{\Lambda_{m F}^{k l}(t)-\Lambda_{m}^{k l}}{\tau}-R_{V} \Lambda_{m}^{k l} \\
& \frac{\mathrm{d} \Lambda^{k l m}}{\mathrm{~d} t}=\sum_{i=1}^{G} \sum_{j=1}^{G} k_{i j} \bar{\omega}_{i j}\left(\Lambda_{i}^{k l} \Lambda_{j}^{m}+\Lambda_{i}^{k m} \Lambda_{j}^{l}+\Lambda_{i}^{k} \Lambda_{j}^{l m}\right) \\
& +\frac{\Lambda_{F}^{k l m}(t)-\Lambda^{k l m}}{\tau}-R_{V} \Lambda^{k l m} .
\end{aligned}
$$

A simpler and computationally more efficient method should be used before gelation. Rate equations for the moments $\lambda_{i j k \ldots}^{m m p}$ are obtained replacing $\sigma=\mathbf{1}_{G}$, $\zeta=1^{N}$ in eqs (40)-(48). As there is no gel, all endgroups belong to finite molecules, and so the following equalities hold:

$$
\begin{aligned}
& A_{k}=\lambda_{k}=\Lambda_{k}\left(1_{G}, 1^{N}\right) \\
& U_{k}=\lambda^{k}=\Lambda^{k}\left(1_{G}, 1^{N}\right)
\end{aligned}
$$

and the moments verify a set of ordinary differential equations given in Table 4.

\section{GEL STRUCTURE AND ELASTIC PROPERTIES}

Several pioneers of polymer science, such as Kuhn, Guth, James, Mark, Flory, Gee and Treloar, have contributed to the theory of rubber elasticity [see Flory (1953) and Treloar (1975)]. However, many essential problems remain incompletely understood in spite of 50 years of research. Even the simpler problem of the prediction or correlation of the small-strain equilibrium shear modulus, $G_{e}$, has no clear-cut solution. Two extreme models are available for describing the network, the affine and the phantom models. In addition, it was once believed that an appreciable contribution to $G_{\mathrm{e}}$ came from the so called "trapped entanglements" (Langley, 1968). In the early eighties, it was generally accepted that the following relation held for $G_{e}$ :

$$
G_{\mathrm{e}}=\left(v_{\mathrm{e}}-h \mu_{\mathrm{e}}\right) R T+G_{N \mathrm{e}}^{0} T_{\mathrm{e}} .
$$

In this equation, $v_{e}$ and $\mu_{e}$ are, respectively, the mole concentrations of elastically active chains (EANC) and junctions (EANJ) (they will be rigorously defined below). The empirical parameter $h$, between 0 and 1 , allows for intermediate behaviour between affine 
Table 4. Mass balance equations for the moments of VNMND in a CSTR before gelation

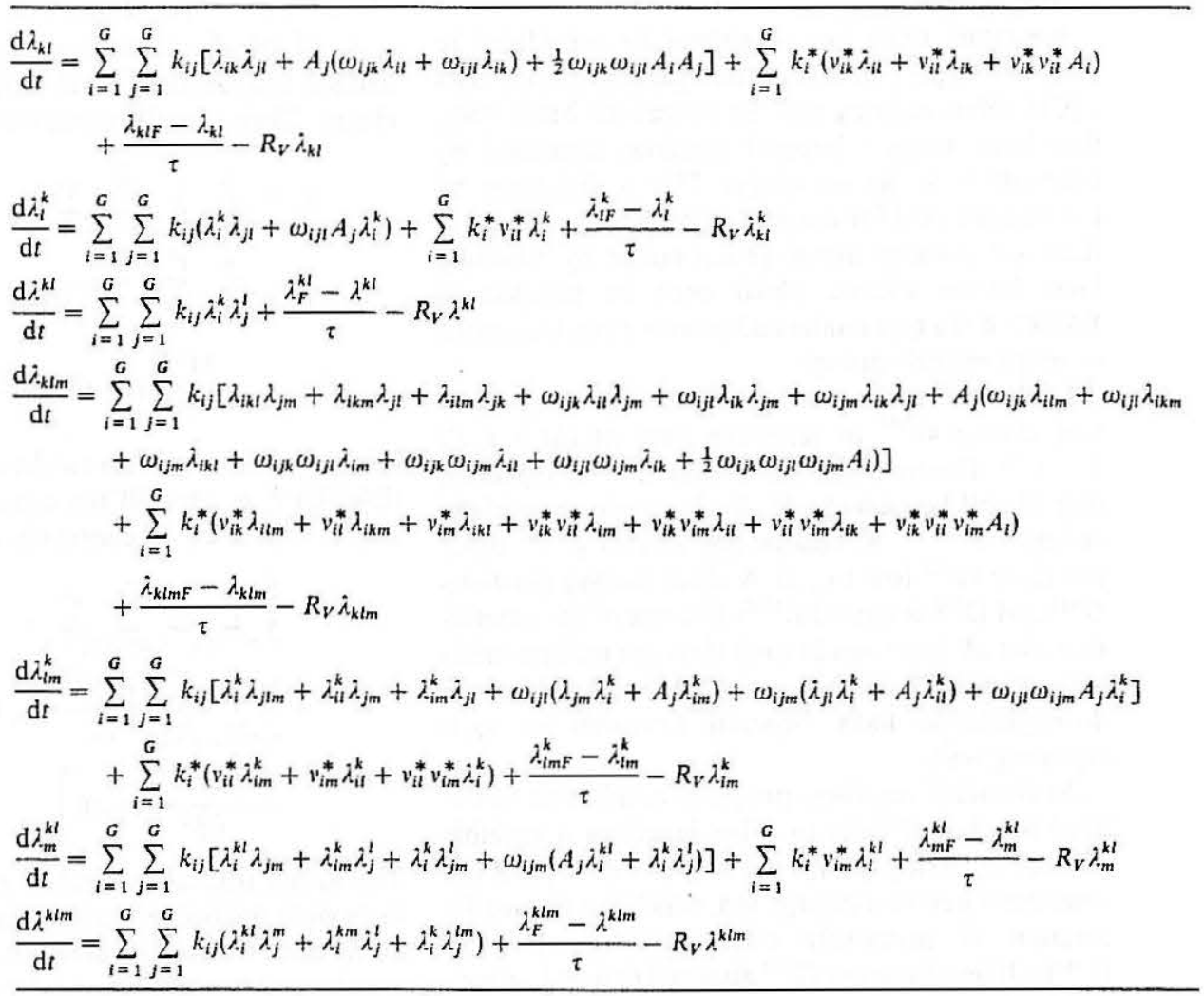

$(h=0)$ and phantom networks $(h=1)$ (Dossin and Graessley, 1979). The term $G_{N e}^{0} T_{e}$ was introduced in order to take into account entanglements between chains. It is the product of a modulus $G_{N e}^{0}$, in principle depending only on the chemical nature of polymer, and of a factor $T_{e}$, representing the fraction of the maximum concentration of topological interactions.

Although at one time it seemed that eq. (59) had a sound experimental basis [see e.g. Pearson and Graessley (1980), Valles and Macosko (1979), Gottlieb et al. (1981) and also Dusek (1986)], Flory and coworkers have criticized the concept of trapped entanglements between chains contributing to network elasticity (Flory and Erman, 1984) and have refined the elasticity theory based on different assumptions (Flory, 1977; Erman and Flory, 1978). Recent experimental and theoretical studies (Lee and Eichinger, 1990) do not support the need of Langley's contribution. Nevertheless, we will discuss briefly how $T_{e}$ can be predicted, after dealing with the much more important problem of predicting the EANJ and EANC concentrations.

Theory of branching processes (TBP) has been used for computing concentrations of EANC and EANJ soon after its introduction (Dobson and Gordon, 1964). The mathematically equivalent "recursive approach" by Macosko and Miller (Miller and Macosko, 1976, 1980) has later been used with more complex chemical systems. This could not render TBP obsolete, and application of TBP to prediction of polymer structure has lately been extended and perfected (Dusek, 1985).

However, TBP describes exactly only systems at chemical equilibrium. Some irreversible batch polycondensations (specially when strict equal reactivity of end-groups holds) lead to the same polymer structure as if a chemical equilibrium prevailed, but this assumption usually breaks down in the presence of substitution effects. No kinetically based treatment was available to cope with this situation. In fact, it was even believed that such a treatment could not be found (Dusek, 1985).

In this paper, the problem of mathematically predicting the concentration of network structures relevant to the prediction of elastic properties is solved for a general irreversible non-linear polymerization, provided that repeating units combine to form treelike molecules. As before, the key information is the set of stoichiometric coefficients of the reactions between reactive groups, which are used for establishing the needed mass balance equations.

\section{INTERNAL JUNCTIONS IN REPEATING UNITS, DISTRIBUTIONS OF JUNCTIONS AND EXTINCTION PROBABILITIES}

Elastic properties of gel are determined by its elastically active junctions (EANJ) which are defined (Scanlan, 1960; Case, 1960; Flory, 1982) as molecular fragments linked to three or more independent network chains (chains going to infinity). Elastically ac- 
tive chains (EANC) are all independent chains connecting EANJ.

Repeating units can sometimes be considered to contain a single junction, such as pentaerithrytol units $\mathrm{C}\left(\mathrm{CH}_{2} \mathrm{OH}\right)_{4}$ reacting with an isocyanate. Most often, they have multiple internal junctions connected by relatively long flexible chains. This is illustrated by polyamines used for curing epoxide resins with more than one primary amine group linked by relatively long flexible chains, which must be counted as EANCs if the two amine end-groups react with three or more epoxide groups.

So, let $J^{M}$ be the number of distinguishable junction classes $\mathrm{G}^{\mathrm{MJ}}$ in repeating unit $M(M=1, N$; $\left.J=J^{M}\right)$. The number of junctions $\mathrm{G}^{\mathrm{MJ}}$ in repeating unit $M$ will be named $\rho^{M J}$. Each junction is initially attached to $f_{i}^{M J} \mathbf{A}_{\mathrm{i}}$ end-groups and to $g^{M J K}$ other junctions $G^{\mathrm{MK}}$ (see Fig. 5). A chain linking junctions $\mathbf{G}^{\mathrm{MJ}}$ and $\mathbf{G}^{\mathrm{MK}}$ is named $\mathrm{L}^{\mathrm{MJK}}$. Because of the assumption that all junctions in each class are indistinguishable, there will be at most $J^{M}\left(J^{M}+1\right) / 2$ kinds of distinguishable links between junctions in each repeating unit.

As chemical reactions progress, in addition to the fixed number of links to other junctions a variable number of linking groups are added to each junction, sometimes new end-groups too, which are formed by reaction of previously existent end-groups. Let $\mathrm{G}^{\mathrm{MJ}}(\mathrm{a}, \mathrm{I})$ be a junction $\mathrm{G}^{\mathrm{MJ}}$ attached to a end-groups and $\mathrm{I}$ linking groups, respectively, and let $G^{M J}(\mathrm{a}, \mathrm{I})$ be its mole concentration, according to our usual convention, $\bar{G}^{M J}(\alpha, \zeta)$ be its vectorial discrete transform,

$$
\begin{aligned}
\bar{G}^{M J}(\alpha, \zeta)= & \sum_{a_{1}=0}^{\infty} \ldots \sum_{a_{G}=0}^{\infty} \sum_{l_{11}=0}^{\infty} \ldots \sum_{l_{G G}=0}^{\infty} \alpha_{1}^{a_{1}} \ldots \alpha_{G}^{a_{G}} \zeta_{11}^{l_{11}} \\
& \ldots \zeta_{G G}^{l_{G G}} G^{M J}\left(a_{1}, \ldots, a_{G}, l_{11}, \ldots, l_{G G}\right) .
\end{aligned}
$$

The elastic properties of the gel are related to the distributions of junctions. Each junction $\mathbf{G}^{\mathbf{M J}}$ is said to have a rank $x=0,1,2$, and so on, according to the number $x$ of network chains which start from it. So, the key quantities for the characterization of gel will be the distributions of numbers of network chains $T^{M J}(\mathrm{a}, x)$ or their generating functions $\bar{T}^{M J}(\alpha, \xi)$.

In what follows, a tree-like structure of the repeating units and of the polymer will be assumed. The extension of this approach to structures containing loops should be possible, but we could as yet find no simple procedure for systematically excluding dangling loops.

For each linking group $\mathrm{L}_{\mathrm{ij}}$ or $\mathrm{L}^{\mathrm{MJK}}$, the fractions of chains which stem from it, in both directions, and do not go to infinity, are the extinction probabilities $v_{i j}$ and $v_{j i}$ (most of the times they are different when $i \neq j)$, or $v^{M J K}$ and $v^{M K J}$. So, the following relation is the base of the present kinetic treatment:

$$
\begin{aligned}
\bar{T}^{M J}(\alpha, \xi)= & \prod_{K=1}^{J^{M I}}\left[v^{M J K}+\xi\left(1-v^{M J K}\right)\right]^{g J K} \\
& \times \bar{G}^{M J}\left[\alpha, \mathrm{v}+\xi\left(\mathbf{1}_{G G}-\mathrm{v}\right)\right] .
\end{aligned}
$$

CONCENTRATIONS OF EANJ, EANC, WEIGHT FRACTION OF PENDANT MATERIAL AND TRAPPING FACTOR

An elastically active network junction (EANJ) is defined as a junction linked to three or more network chains. Their overall concentration, $\mu_{e}$, is therefore

$$
\begin{aligned}
\mu_{e}= & \sum_{M=1}^{N} \sum_{J=1}^{J^{M}} \sum_{x=3}^{\infty} \sum_{a_{1}=0}^{\infty} \ldots \sum_{a_{G}=0}^{\infty} T^{M J}(\mathrm{a}, x) \\
= & \sum_{M=1}^{N} \sum_{J=1}^{J^{M}}\left[\bar{T}^{M J}\left(\mathbf{1}_{G}, 1\right)-\bar{T}^{M J}\left(\mathbf{1}_{G}, 0\right)\right. \\
& \left.-\frac{\partial \bar{T}^{M J}}{\partial \xi}\left(\mathbf{1}_{G}, 0\right)-\frac{1}{2} \frac{\partial^{2} \bar{T}^{M J}}{\partial \xi^{2}}\left(\mathbf{1}_{G}, 0\right)\right] .
\end{aligned}
$$

The concentration of elastically active network chains (EANC) $v_{e}$ is one-half the concentration of network chains carried by all elastically active junctions:

$$
\begin{aligned}
v_{e}= & \frac{1}{2} \sum_{M=1}^{N} \sum_{J=1}^{J^{M}} \sum_{x=3}^{\infty} \sum_{a_{1}=0}^{\infty} \ldots \sum_{a_{G}=0}^{\infty} x T^{M J}(\mathrm{a}, x) \\
= & \frac{1}{2} \sum_{M=1}^{N} \sum_{J=1}^{J^{M}}\left[\frac{\partial \bar{T}^{M J}}{\partial \xi}\left(\mathbf{1}_{G}, 1\right)-\frac{\partial \bar{T}^{M J}}{\partial \xi}\left(\mathbf{1}_{G}, 0\right)\right. \\
& \left.-\frac{\partial^{2} \bar{T}^{M J}}{\partial \xi^{2}}\left(\mathbf{1}_{G}, 0\right)\right] .
\end{aligned}
$$

The weight fraction of pendant material in gel, $w_{P}$, is the weight fraction of material linked to the gel by no more than two active network chains. The sum of $w_{P}$ with weight fraction of $\operatorname{sol} w_{s}$ is then

$$
\begin{aligned}
w_{P}+w_{s}= & \sum_{M=1}^{N} \sum_{J=1}^{J^{M}} \sum_{a_{1}=0}^{\infty} \ldots \sum_{a_{G}=0}^{\infty}\left(\bar{M}_{n}^{M J}+a_{1} M_{1}\right. \\
& \left.+\cdots+a_{G} M_{G}\right)\left[T^{M J}\left(a_{1}, \ldots, a_{G}, 0\right)\right. \\
& +T^{M J}\left(a_{1}, \ldots, a_{G}, 1\right) \\
& \left.+T^{M J}\left(a_{1}, \ldots, a_{G}, 2\right)\right] \\
= & \sum_{M=1}^{N} \sum_{J=1}^{J^{N}}\left\{\overline { M } _ { n } ^ { M J } \left[\bar{T}^{M J}\left(\mathbf{1}_{G}, 0\right)\right.\right. \\
& \left.+\frac{\partial \bar{T}^{M J}}{\partial \xi}\left(\mathbf{1}_{G}, 0\right)+\frac{1}{2} \frac{\partial^{2} \bar{T}^{M J}}{\partial \bar{\xi}^{2}}\left(\mathbf{1}_{G}, 0\right)\right] \\
& +M_{1}\left[\frac{\partial \bar{T}^{M J}}{\partial \alpha_{1}}\left(\mathbf{1}_{G}, 0\right)+\frac{\partial^{2} \bar{T}^{M J}}{\partial \alpha_{1} \partial \xi}\left(\mathbf{1}_{G}, 0\right)\right. \\
& \left.+\frac{1}{2} \frac{\partial^{3} \bar{T}^{M J}}{\partial \alpha_{1} \partial \bar{\zeta}^{2}}\left(\mathbf{1}_{G}, 0\right)\right]+\cdots \\
& +M_{G}\left[\frac{\partial \bar{T}^{M J}}{\partial \alpha_{G}}\left(\mathbf{1}_{G}, 0\right)+\frac{\partial^{2} \bar{T}^{M J}}{\partial \alpha_{G} \partial \xi}\left(\mathbf{1}_{G}, 0\right)\right. \\
& \left.\left.+\frac{1}{2} \frac{\partial^{3} \bar{T}^{M J}}{\partial \alpha_{G} \partial \xi^{2}}\left(\mathbf{1}_{G}, 0\right)\right]\right\} .
\end{aligned}
$$

In writing eq. (64), the molecular weight of each repeating unit was assumed to be shared by its junctions. $\bar{M}_{n}^{M J}$ is the number-average molecular weight allocated to junction $G^{\mathrm{MJ}}$. No molecular weight was allocated to links between junctions.

The original definition of Langley's trapping factor $T_{e}$ must be generalized, so as to encompass any network. $T_{\mathrm{e}}$ is proportional to the product of probabili- 
ties of contacts between repeating units linked to the network by two or more network chains. We will define a non-normalized $T_{e}^{*}$ as the square of weight fractions of material linked to the gel to two or more network chains (volume fractions could also be introduced). Consequently, $T_{e}$ is $T_{e}^{*}$ divided by its maximum possible value, $T_{\text {eMAX }}^{*}$, which can often be taken as the value for exact stoichiometric balance. Therefore, we write

$$
\begin{aligned}
\sqrt{T_{e}^{*}}= & \sqrt{T_{e} T_{e M A X}^{*}}=1-\sum_{M=1}^{N} \sum_{J=1}^{J} \sum_{a_{1}=0}^{\infty} \ldots \sum_{a_{G}=0}^{\infty} \\
& \times\left(\bar{M}_{n}^{M J}+a_{1} M_{1}+\cdots+a_{G} M_{G}\right) \\
& \times\left[T^{M J}(\mathbf{a}, 0)+T^{M J}(\mathrm{a}, 1)\right] \\
= & 1-\sum_{M=1}^{N} \sum_{J=1}^{J^{M}}\left\{\overline { M } _ { n } ^ { M J } \left[\bar{T}^{M J}\left(\mathbf{1}_{G}, 0\right)\right.\right. \\
& \left.+\frac{\partial \bar{T}^{M J}}{\partial \xi}\left(\mathbf{1}_{G}, 0\right)\right]+M_{1}\left[\frac{\partial \bar{T}^{M J}}{\partial \alpha_{1}}\left(\mathbf{1}_{G}, 0\right)\right. \\
& \left.+\frac{\partial^{2} \bar{T}^{M J}}{\partial \alpha_{1} \partial \xi}\left(\mathbf{1}_{G}, 0\right)\right]+\cdots+M_{G}\left[\frac{\partial \bar{T}^{M J}}{\partial \alpha_{G}}\left(\mathbf{1}_{G}, 0\right)\right. \\
& \left.\left.+\frac{\partial^{2} \bar{T}^{M J}}{\partial \alpha_{G} \partial \xi}\left(\mathbf{1}_{G}, 0\right)\right]\right\} .
\end{aligned}
$$

Taking into account eq. (61), the selected gel properties are related to the $\bar{G}^{M J}$ and their derivatives by

$$
\begin{aligned}
& \mu_{c}=\sum_{M=1}^{N} \sum_{J=1}^{J^{M}}\left[\bar{G}^{M J}\left(\mathbf{1}_{G}, \mathbf{1}_{G G}\right)\right. \\
& -\left(H^{M J}+H^{M J}+\frac{1}{2} H^{M J *}\right) \bar{G}^{M J}\left(\mathbf{1}_{G}, \mathbf{v}\right) \\
& -\left(H^{M J}+H^{M J^{\prime}}\right) \sum_{i=1}^{G} \sum_{j=1}^{G}\left(1-v_{i j}\right) \Gamma^{M J i j}\left(1_{G}, \mathrm{v}\right) \\
& -\frac{1}{2} H^{M J} \sum_{i=1}^{G} \sum_{j=1}^{G} \sum_{k=1}^{G} \sum_{l=1}^{G}\left(1-v_{i j}\right) \\
& \left.\times\left(1-v_{k l}\right) \Gamma^{M J i j k l}\left(\mathbf{1}_{G}, \mathbf{v}\right)\right] \\
& v_{e}=\frac{1}{2} \sum_{M=1}^{N} \sum_{J=1}^{J^{M}}\left\{I^{M J} \bar{G}^{M J}\left(\mathbf{1}_{G}, \mathbf{1}_{G G}\right)\right. \\
& -\left(H^{M J^{\prime}}+H^{M J^{\prime \prime}}\right) \bar{G}^{M J}\left(\mathbf{1}_{G}, \mathrm{v}\right) \\
& +\sum_{i=1}^{G} \sum_{j=1}^{G}\left(1-v_{i j}\right)\left[\Gamma^{M J i j}\left(\mathbf{1}_{G}, \mathbf{1}_{G G}\right)\right. \\
& \left.-\left(H^{M J}+2 H^{M J^{\prime}}\right) \Gamma^{M J i j}\left(1_{G}, \mathrm{v}\right)\right] \\
& -H^{M J} \sum_{i=1}^{G} \sum_{j=1}^{G} \sum_{k=1}^{G} \sum_{i=1}^{G}\left(1-v_{i j}\right) \\
& \left.\times\left(1-v_{k l}\right) \Gamma^{M J i j k l}\left(\mathbf{1}_{G}, v\right)\right\} \\
& w_{P}+w_{s}=\sum_{M=1}^{N} \sum_{J=1}^{J^{M}}\left\{\overline { M } _ { n } ^ { M J } \left[\left(H^{M J}+H^{M J}\right.\right.\right. \\
& \left.+\frac{1}{2} H^{M J^{\prime \prime}}\right) \bar{G}^{M J}\left(\mathbf{1}_{G}, \mathrm{v}\right)+\left(H^{M J}+H^{M J}\right) \\
& \times \sum_{i=1}^{G} \sum_{j=1}^{G}\left(1-v_{i j}\right) \Gamma^{M J i j}\left(\mathbf{1}_{G}, \mathbf{v}\right)
\end{aligned}
$$

$$
\begin{aligned}
& +\frac{1}{2} H^{M J^{\prime \prime}} \sum_{i=1}^{G} \sum_{j=1}^{G} \sum_{k=1}^{G} \sum_{i=1}^{G}\left(1-v_{i j}\right) \\
& \left.\times\left(1-v_{k l}\right) \Gamma^{M J i j k l}\left(\mathbf{1}_{G}, \mathbf{v}\right)\right] \\
& +\sum_{m=1}^{G} M_{m}\left[\Gamma_{m}^{M J}\left(\mathbf{1}_{G}, \mathbf{v}\right)\right. \\
& +\sum_{i=1}^{G} \sum_{j=1}^{G}\left(1-v_{i j}\right) \Gamma_{m}^{M J i j}\left(1_{G}, v\right) \\
& +\frac{1}{2} \sum_{i=1}^{G} \sum_{j=1}^{G} \sum_{k=1}^{G} \sum_{l=1}^{G}\left(1-v_{i j}\right) \\
& \left.\left.\times\left(1-v_{k l}\right) \Gamma_{m}^{M S i j k l}\left(1_{G}, v\right)\right]\right\} \\
& \sqrt{T_{e}^{*}}=1-\sum_{M=1}^{N} \sum_{J=1}^{J^{M}} \\
& \times\left\{\overline { M } _ { n } ^ { M J } \left[\left(H^{M J}+H^{M J^{\prime}}\right) \bar{G}^{M}\left(\mathbf{1}_{G}, \mathbf{v}\right)\right.\right. \\
& \left.+H^{M J} \sum_{i=1}^{G} \sum_{j=1}^{G}\left(1-v_{i j}\right) \Gamma^{M J i j}\left(\mathbf{1}_{G}, \mathbf{v}\right)\right] \\
& +\sum_{m=1}^{G} M_{m}\left[\Gamma_{m}^{M J}\left(1_{G}, v\right)\right. \\
& \left.\left.+\sum_{i=1}^{G} \sum_{j=1}^{G}\left(1-v_{i j}\right) \Gamma_{m}^{M J i j}\left(1_{G}, v\right)\right]\right\}
\end{aligned}
$$

where

$$
\begin{aligned}
\Gamma_{m n n}^{M J_{i j k l \ldots} \ldots}= & \frac{\partial \partial \ldots \partial \partial \ldots \bar{G}^{M J}}{\partial \zeta_{i j} \partial \zeta_{k i} \ldots \partial \log \alpha_{m} \partial \log \alpha_{n} \ldots}(\alpha, \zeta) \\
H^{M J}= & \prod_{K=1}^{J^{M}}\left(v^{M J K}\right)^{3 / J K} \\
H^{M J^{\prime}}= & H^{M J} \sum_{K=1}^{J^{M}} g^{M J K} \frac{1-v^{M J K}}{v^{M J K}} \\
H^{M J^{\prime \prime}}= & H^{M J}\left[\left(\sum_{K=1}^{J^{M}} g^{M J K} \frac{1-v^{M J K}}{v^{M J K}}\right)^{2}\right. \\
& \left.-\sum_{K=1}^{J^{M}} g^{M J K} \frac{\left(1-v^{M J K}\right)^{2}}{\left(v^{M J K}\right)^{2}}\right] \\
I^{M J}= & \sum_{K^{\prime}=1}^{J^{M}} g^{M J K}\left(1-v^{M J K}\right) .
\end{aligned}
$$

\section{RATE EQUATIONS FOR PREDICTING GEL PROPERTIES}

The concentrations of linking groups $\mathbf{L}_{\mathrm{ij}}$ need to be predicted. Regardless of whether they belong to the gel or not, their rate of formation is simply

$$
R_{\mathrm{L}_{i j}}=k_{i j} A_{i} A_{j} .
$$

The extinction probabilities can be computed, without the need of invoking TBP, by following the concentrations of dangling chains $\mathrm{X}_{\mathrm{ij}}(\mathrm{a})$ which start at an $\mathrm{L}_{\mathrm{ij}}$, go towards the repeating unit previously carrying the end-group $A_{j}$ and lead finally to a end-groups of different kinds. Each extinction probability $v_{i j}$ is simply the fraction of those fragments which are finite, 
regardless of the numbers of their end-groups:

$$
v_{i j}=\sum_{a_{1}=0}^{\infty} \ldots \sum_{a_{G}=0}^{\infty} X_{i j}\left(a_{1}, \ldots, a_{G}\right) / L_{i j} .
$$

Introducing the vectorial discrete transform

$\bar{X}_{i j}(\sigma)=\sum_{a_{1}=0}^{\infty} \ldots \sum_{a_{G}=0}^{\infty} \sigma_{1}^{a_{1}} \ldots \sigma_{G}^{a_{G}} X_{i j}\left(a_{1}, \ldots, a_{G}\right)$

eq. (76) becomes

$$
v_{i j}=\bar{X}_{i j}\left(\mathbf{1}_{G}\right) / L_{i j} .
$$

A rate equation for $X_{k l}(a)$ can easily be written, realizing that an existent $\mathbf{X}_{\mathbf{k l}}(\mathbf{a})$ grows by reaction of its end-groups with $\mathbf{P}(\mathbf{a}, \mathbf{n})$ or by non-intermolecular reaction of a larger fragment, is destroyed by reaction of its a end-groups and is created whenever an endgroup $\mathbf{A}_{\mathbf{k}}$ reacts intermolecularly with an end-group $\mathbf{A}_{1}$ belonging to a polymer molecule $\mathbf{P}(\mathbf{a}, \mathbf{n})$ :

$$
\begin{aligned}
\bar{R}_{\mathbf{X}_{k l}}(\sigma)= & \sum_{i=1}^{G} \sum_{j=1}^{G} k_{i j} \frac{\partial \bar{X}_{k l}}{\partial \log \sigma_{i}}\left(\bar{\omega}_{i j} \frac{\partial \bar{P}}{\partial \log \sigma_{j}}-A_{j}\right) \\
& +\sum_{i=1}^{G} k_{i}^{*} \frac{\partial \bar{X}_{k l}}{\partial \log \sigma_{i}}\left(\bar{v}_{i}^{*}-1\right) \\
& +k_{k l} A_{k} \bar{v}_{l k} \frac{\partial \bar{P}}{\partial \log \sigma_{l}} .
\end{aligned}
$$

Coming now to the links between junctions, their rate of formation is obviously nil and so their concentration is easily determined by knowing the concentrations of repeating units:

$$
L^{M I J}=\rho^{M I} g^{M I J} U^{M} .
$$

Similarly, it is necessary to introduce the concentrations of dangling chains $\mathrm{X}^{\mathrm{M} / \mathrm{s}}$ (a) which start at a $\mathrm{G}^{\mathrm{MI}}$ junction and go towards a junction $\mathbf{G}^{\mathrm{MJ}}$, leading to a end-groups of different kinds. Extinction probability $v^{M I J}$ is the fraction of those fragments which are finite, regardless of the numbers of their end-groups:

$v^{M I J}=\sum_{a_{1}=0}^{\infty} \ldots \sum_{a_{G}=0}^{\infty} X^{M I J}\left(a_{1}, \ldots, a_{G}\right) / L^{M I J}$.

The rate law for $\mathbf{X}^{M 1 J}$ (a) is identical to eq. (79), except for the absence of the term coming from the creation of new links:

$$
\begin{aligned}
\bar{R}_{\mathbf{X}^{\text {MKL. }}}(\sigma)= & \sum_{i=1}^{G} \sum_{j=1}^{G} k_{i j} \frac{\partial \bar{X}^{M K L}}{\partial \log \sigma_{i}}\left(\bar{\omega}_{i j} \frac{\partial \bar{P}}{\partial \log \sigma_{j}}-A_{j}\right) \\
& +\sum_{i=1}^{G} k_{i}^{*} \frac{\partial \bar{X}^{M K L}}{\partial \log \sigma_{i}}\left(\bar{v}_{i}^{*}-1\right) .
\end{aligned}
$$

Inserting rate laws (79) or (82) in the relevant mass balance equations of the reactor, partial differential equations are obtained. They must be solved simultaneously with the mass balance equation for the discrete transform of VNMND, $\bar{P}(\sigma, \zeta)$, for $\zeta=1^{N}$ [eqs (27)-(32)]. In the case of the CSTR, those equations are first-order and semi-linear:

$\frac{\partial \bar{X}_{k l}}{\partial t}=\sum_{i=1}^{G} \sum_{j=1}^{G} k_{i j} \frac{\partial \bar{X}_{k l}}{\partial \log \sigma_{i}}\left(\bar{\omega}_{i j} \frac{\partial \bar{P}}{\partial \log \sigma_{j}}-A_{j}\right)$

$$
\begin{gathered}
+\sum_{i=1}^{G} k_{i}^{*} \frac{\partial \bar{X}_{k l}}{\partial \log \sigma_{i}}\left(\bar{v}_{i}^{*}-1\right)+k_{k l} A_{k} \bar{v}_{l k} \frac{\partial \bar{P}}{\partial \log \sigma_{l}} \\
+\frac{\bar{X}_{k l F}(t)-\bar{X}_{k l}}{\tau}-R_{V} \bar{X}_{k l} \\
\left.\bar{X}_{k l}\right|_{t=0}=\bar{X}_{k l 0}(\sigma)
\end{gathered}
$$

Adding the $G^{2}$ equations (83) to eq. (20) does not change the characteristics $\sigma(t)$, which are computed using eqs (27)-(35) as before. Along the characteristics, $\bar{X}_{k l}$ and $\bar{X}^{M K L}$ are determined by integrating

$$
\begin{gathered}
\frac{\mathrm{d} \bar{X}_{k l}}{\mathrm{~d} t}=k_{k l} A_{k} \bar{v}_{l k} \Lambda_{l}+\frac{\bar{X}_{k l F}(t)-\bar{X}_{k l}}{\tau}-R_{V} \bar{X}_{k l} \\
\frac{\mathrm{d} \bar{X}^{M K L}}{\mathrm{~d} t}=\frac{\bar{X}_{F}^{M K L}(t)-\bar{X}^{M K L}}{\tau}-R_{V} \bar{X}^{M K L} .
\end{gathered}
$$

Before the gel point, $\bar{X}_{k l}\left(1_{G}\right)=L_{k l}$, since $\sigma(t)=1_{G}$ along the characteristic that ends at $\mathbf{1}_{G}$, and (82) degenerates into the rate law for $\mathbf{L}_{\mathrm{kl}}$, equation (75).

It remains now to establish rate laws for the $\bar{G}^{M I}$ and their derivatives $\Gamma_{m n \ldots}^{M l i j k l \ldots}$ in order to compute the right-hand sides of eqs (66)-(69). This is also easily done, since

$$
\begin{aligned}
& \mathbf{G}^{\mathrm{MI}}(\mathbf{a}, \mathbf{l})+\mathbf{A}_{\mathbf{j}} \\
& \quad \stackrel{a_{i} k_{i j}}{\longrightarrow} \mathrm{G}^{\mathrm{MI}}\left(\mathbf{a}+v_{i j}, l_{11}, \ldots, l_{i j}+1, \ldots, l_{G G}\right)
\end{aligned}
$$

and therefore

$$
\begin{aligned}
\bar{R}_{\mathrm{G}}^{\mathrm{MI}}(\alpha, \zeta)= & \sum_{i=1}^{G} \sum_{j=1}^{G} k_{i j} \frac{\partial \bar{G}^{M I}}{\partial \log \alpha_{i}} A_{j}\left[\zeta_{i j} \bar{v}_{i j}(\alpha)-1\right] \\
& +\sum_{i=1}^{G} k_{i}^{*} \frac{\partial \bar{G}^{M I}}{\partial \log \alpha_{i}}\left[\bar{v}_{i}^{*}(\alpha)-1\right] .
\end{aligned}
$$

Differentiation of eq. (87) leads to the rate laws of the $\Gamma_{m n \ldots}^{M I j i k l \cdots}$ :

$$
\begin{aligned}
R_{\Gamma_{k}^{M I}}= & \sum_{i=1}^{G} \sum_{j=1}^{G} k_{i j} A_{j}\left[\frac{\partial \Gamma_{k}^{M I}}{\partial \log \alpha_{i}}\left(\zeta_{i j} \bar{v}_{i j}-1\right)\right. \\
& \left.+\zeta_{i j} \bar{v}_{i j} v_{i j k} \Gamma_{i}^{M I}\right] \\
& +\sum_{i=1}^{G} k_{i}^{*}\left[\frac{\partial \Gamma_{k}^{M I}}{\partial \log \alpha_{i}}\left(\bar{v}_{i}^{*}-1\right)+\bar{v}_{i}^{*} v_{i k}^{*} \Gamma_{i}^{M I}\right] \\
R_{\Gamma^{M I M I}}= & \sum_{i=1}^{G} \sum_{j=1}^{G} k_{i j} \frac{\partial \Gamma^{M I k l}}{\partial \log \alpha_{i}} A_{j}\left(\zeta_{i j} \bar{v}_{i j}-1\right) \\
& +\sum_{i=1}^{G} k_{i}^{*} \frac{\partial \Gamma^{M I k l}}{\partial \log \alpha_{i}}\left(\bar{v}_{i}^{*}-1\right)+k_{k l} \bar{v}_{k l} \Gamma_{k}^{M I} A_{l} \\
k_{\Gamma_{k l}^{M I}=} & \sum_{i=1}^{G} \sum_{j=1}^{G} k_{i j} A_{j}\left[\frac{\partial \Gamma_{k l}^{M I}}{\partial \log \alpha_{i}}\left(\zeta_{i j} \bar{v}_{i j}-1\right)\right. \\
& \left.+\zeta_{i j} \bar{v}_{l j}\left(v_{i j l} \Gamma_{k i}^{M I}+v_{i j k} \Gamma_{l i}^{M I}+v_{i j k} v_{i j l} \Gamma_{i}^{M I}\right)\right] \\
& +\sum_{i=1}^{G} k_{i}^{*}\left[\frac{\partial \Gamma_{k l}^{M I}}{\partial \log \alpha_{i}}\left(\bar{v}_{i}^{*}-1\right)\right. \\
& \left.+\bar{v}_{i}^{*} v_{i k}^{*}\left(\Gamma_{i l}^{M I}+v_{i l}^{*} \Gamma_{i}^{M I}\right)\right]
\end{aligned}
$$




$$
\begin{aligned}
R_{\Gamma_{m}^{M M l}}= & \sum_{i=1}^{G} \sum_{j=1}^{G} k_{i j} A_{j}\left[\frac{\partial \Gamma_{m}^{M I k l}}{\partial \log \alpha_{i}}\left(\zeta_{i j} \bar{v}_{i j}-1\right)\right. \\
& \left.+\zeta_{i j} \bar{v}_{i j} v_{i j m} \Gamma_{i}^{M I k l}\right] \\
& +\sum_{i=1}^{G} k_{i}^{*}\left[\frac{\partial \Gamma_{m}^{M I k l}}{\partial \log \alpha_{i}}\left(\bar{v}_{i}^{*}-1\right)+\bar{v}_{i}^{*} v_{i m}^{*} \Gamma_{i}^{M I k l}\right] \\
& +k_{k l} A_{l} \bar{v}_{k l}\left(\Gamma_{m k}^{M I}+v_{k l m} \Gamma_{k}^{M I}\right) \\
R_{\Gamma^{M M n t m m}=} & \sum_{i=1}^{G} \sum_{j=1}^{G} k_{i j} A_{j} \frac{\partial \Gamma^{M I k l m n}}{\partial \log \alpha_{i}}\left(\zeta_{i j} \bar{v}_{i j}-1\right) \\
& +\sum_{i=1}^{G} k_{i}^{*} \frac{\partial \Gamma^{M I k l m n}}{\partial \log \alpha_{i}}\left(\bar{v}_{i}^{*}-1\right) \\
& +k_{k l} A_{l} \bar{v}_{k l} \Gamma_{k}^{M I m n}+k_{m n} A_{n} \bar{v}_{m n} \Gamma_{m}^{M I k l} .
\end{aligned}
$$

Inserting these rate laws in the mass balance in a CSTR, a system of first-order semi-linear partial differential equations is obtained. Its characteristics verify

$$
\begin{aligned}
\frac{\mathrm{d} \log \alpha_{k}}{\mathrm{~d} t} & =\sum_{i=1}^{G} k_{k i} A_{i}\left(1-\zeta_{k i} \bar{v}_{k i}\right)+k_{k}^{*}\left(1-\bar{v}_{k}^{*}\right) \\
\left.\alpha_{k}\right|_{t=0} & =\alpha_{k 0} .
\end{aligned}
$$

In order to pass through a previously specified value of $\alpha$ at a time $t$ for a certain matrix $\zeta$, a system of $G$ algebraic equations must be solved to find the starting vector $\alpha_{0}$ :

$$
\log \alpha_{k}\left(\log \alpha_{0}, \zeta\right)-\log \alpha_{k}=0
$$

Just as before, Newton-Raphson's method can be used to solve this system of algebraic equations, choosing small enough starting values for $\alpha_{0}$. The Jacobian of system (95),

$$
Z_{k l}=\frac{\partial \log \alpha_{k}}{\partial \log \alpha_{l 0}}
$$

can be found by solving eq. (93) simultaneously with the additional $G^{2}$ ordinary differential equations:

$$
\begin{aligned}
\frac{\mathrm{d} Z_{k l}}{\mathrm{~d} t}= & -\sum_{i=1}^{G} k_{k i} A_{i} \zeta_{k i} \bar{v}_{k i} \sum_{j=1}^{G} v_{k i j} Z_{j t} \\
& -k_{k}^{*} \bar{v}_{k}^{*} \sum_{j=1}^{G} v_{k j}^{*} Z_{k j,} \\
\left.Z_{k l}\right|_{t=0}= & \begin{cases}1 & \text { if } k=l \\
0 & \text { otherwise. }\end{cases}
\end{aligned}
$$

After computing the starting vector $\alpha_{0}$, the remaining differential equations for computing $\bar{G}^{M I}$ and its derivatives along the characteristics can be simultaneously integrated until the final value of $\alpha$ :

$$
\begin{gathered}
\frac{\mathrm{d} \bar{G}^{M I}}{\mathrm{~d} t}=\frac{\bar{G}_{F}^{M I}(t)-\bar{G}^{M I}}{\tau}-R_{Y} \bar{G}^{M I} \\
\frac{\mathrm{d} \Gamma_{k}^{M I}}{\mathrm{~d} t}=\sum_{i=1}^{G} \sum_{j=1}^{G} k_{i j} \zeta_{i j} \bar{v}_{i j} v_{i j k} A_{j} \Gamma_{i}^{M I}+\sum_{i=1}^{G} k_{i}^{*} \bar{v}_{i}^{*} v_{i k}^{*} \Gamma_{i}^{M I} \\
+\frac{\Gamma_{k F}^{M I}(t)-\Gamma_{k}^{M I}}{\tau}-R_{V} \Gamma_{k}^{M I}
\end{gathered}
$$

$$
\begin{aligned}
& \frac{\mathrm{d} \Gamma^{M I k l}}{\mathrm{~d} t}=k_{k l} \bar{v}_{k l} \Gamma_{k}^{M I} A_{l}+\frac{\Gamma_{F}^{M I k l}(t)-\Gamma^{M I k l}}{\tau}-R_{V} \Gamma^{M l k l} \\
& \frac{\mathrm{d} \Gamma_{k l}^{M I}}{\mathrm{~d} t}=\sum_{i=1}^{G} \sum_{j=1}^{G} k_{i j} A_{j} \zeta_{i j} \vec{v}_{i j} \\
& \times\left(v_{i j l} \Gamma_{k i}^{M I}+v_{i j k} \Gamma_{i i}^{M I}+v_{i j k} v_{i j l} \Gamma_{i}^{M I}\right) \\
& +\sum_{i=1}^{G} k_{i}^{*} \bar{v}_{i}^{*} v_{i k}^{*}\left(\Gamma_{i I}^{M I}+v_{i l}^{*} \Gamma_{i}^{M I}\right) \\
& +\frac{\Gamma_{k l F}^{M I}(t)-\Gamma_{k I}^{M I}}{\tau}-R_{V} \Gamma_{k I}^{M I} \\
& \frac{\mathrm{d} \Gamma_{m}^{M I k l}}{\mathrm{~d} t}=\sum_{i=1}^{G} \sum_{j=1}^{G} k_{i j} A_{j} \zeta_{i j} \bar{v}_{i j} v_{i j m} \Gamma_{i}^{M I k l} \\
& +\sum_{i=1}^{G} k_{i}^{*} \bar{v}_{i}^{*} \bar{v}_{i m}^{*} \Gamma_{i}^{M I k l} \\
& +k_{k l} A_{l} \bar{v}_{k l}\left(\Gamma_{m k}^{M I}+v_{k l m} \Gamma_{k}^{M I}\right) \\
& +\frac{\Gamma_{m F}^{M I k l}(t)-\Gamma_{m}^{M I k l}}{\tau}-R_{V} \Gamma_{m}^{M I k !} \\
& \frac{\mathrm{d} \Gamma^{M I k l m n}}{\mathrm{~d} t}=k_{k l} A_{1} \bar{v}_{k l} \Gamma_{k}^{M I m n}+k_{m n} A_{n} \bar{v}_{m n} \Gamma_{m}^{M I k l} \\
& +\frac{\Gamma_{F}^{M I k l m n}-\Gamma^{M I k l m n}}{\tau}-R_{V} \Gamma^{M I k l m n}
\end{aligned}
$$

\section{THE ALTERNATING POLYCONDENSATION OF TWO POINT-LIKE MONOMERS}

Since this kinetic treatment is entirely new, we illustrate it with the analysis of the irreversible alternating polycondensation of two point-like monomers $U^{1}\left(A_{1}\right)_{f_{1}}$ and $U^{2}\left(A_{2}\right)_{f_{2}}$ in a batch reactor, for which an analytical solution can be found.

There are no reactions consuming end-groups without forming links $\left(v_{i j}^{*}=0\right.$ for all $\left.i, j\right)$. The discrete transform of the matrix of the stoichiometric coefficients of reactions forming links is

$$
\left[\bar{v}_{i j}\right]=\left[\begin{array}{cc}
0 & 1 / \sigma_{1} \\
1 / \sigma_{2} & 0
\end{array}\right] .
$$

Mass balance equations for end-groups, linking groups, polymer molecules and dangling chains in a batch reactor without volume changes can be written as

$$
\begin{aligned}
\frac{\mathrm{d} A_{1}}{\mathrm{~d} t}= & \frac{\mathrm{d} A_{2}}{\mathrm{~d} t}=-\frac{\mathrm{d} L_{12}}{\mathrm{~d} t}=-k_{12} A_{1} A_{2} \\
k_{12}^{-1} \frac{\partial \bar{P}}{\partial t}= & \frac{1}{\sigma_{1} \sigma_{2}} \frac{\partial \bar{P}}{\partial \log \sigma_{1}} \frac{\partial \bar{P}}{\partial \log \sigma_{2}} \\
& -A_{1} \frac{\partial \bar{P}}{\partial \log \sigma_{2}}-A_{2} \frac{\partial \bar{P}}{\partial \log \sigma_{1}} \\
k_{12}^{-1} \frac{\partial \bar{X}_{12}}{\partial t}= & \frac{\partial \bar{X}_{12}}{\partial \log \sigma_{1}}\left(\frac{1}{\sigma_{1} \sigma_{2}} \frac{\partial \bar{P}}{\partial \log \sigma_{2}}-A_{2}\right) \\
& +\frac{\partial \bar{X}_{12}}{\partial \log \sigma_{2}}\left(\frac{1}{\sigma_{1} \sigma_{2}} \frac{\partial \bar{P}}{\partial \log \sigma_{1}}-A_{1}\right)
\end{aligned}
$$




$$
\begin{aligned}
& +\frac{A_{1}}{\sigma_{2}} \frac{\partial \bar{P}}{\partial \log \sigma_{2}} \\
k_{12}^{-1} \frac{\partial \bar{X}_{21}}{\partial t}= & \frac{\partial \bar{X}_{21}}{\partial \log \sigma_{1}}\left(\frac{1}{\sigma_{1} \sigma_{2}} \frac{\partial \bar{P}}{\partial \log \sigma_{2}}-A_{2}\right) \\
& +\frac{\partial \bar{X}_{21}}{\partial \log \sigma_{2}}\left(\frac{1}{\sigma_{1} \sigma_{2}} \frac{\partial \bar{P}}{\partial \log \sigma_{1}}-A_{1}\right) \\
& +\frac{A_{2}}{\sigma_{1}} \frac{\partial \bar{P}}{\partial \log \sigma_{1}} .
\end{aligned}
$$

$$
\begin{aligned}
\frac{\Lambda_{2}}{\sigma_{2} A_{2}}= & \frac{\Lambda_{20}}{\sigma_{20} A_{20}}=\zeta^{2} \sigma_{20}^{f_{2}-1} \\
\bar{P}= & \bar{P}_{t=0}-\frac{\Lambda_{10} \Lambda_{20}}{\sigma_{10} \sigma_{20} A_{10} A_{20}}\left(A_{10}-A_{1}\right) \\
= & U^{1} \zeta^{1} \sigma_{10}^{f_{1}}+U^{2} \zeta^{2} \sigma_{20}^{f_{2}} \\
& -p_{1} f_{1} U^{1} \zeta^{1} \zeta^{2} \sigma_{10}^{f_{1}-1} \sigma_{20}^{f_{2}-1} \\
\bar{X}_{12}= & \frac{\Lambda_{20}}{\sigma_{20} A_{20}}\left(A_{20}-A_{2}\right)=p_{2} A_{20} \sigma_{20}^{f_{2}-1} \\
\bar{X}_{21}= & \frac{\Lambda_{10}}{\sigma_{10} A_{10}}\left(A_{10}-A_{1}\right)=p_{1} A_{10} \sigma_{10}^{f_{1}-1} .
\end{aligned}
$$

The starting point of a characteristic passing through prescribed values of $\sigma_{1}, \sigma_{2}, \zeta^{1}$ and $\zeta^{2}$, defined by $\sigma_{10}$ and $\sigma_{20}$, must be obtained by solving the two coupled algebraic equations

$$
\begin{aligned}
\sigma_{10} & =\sigma_{1}\left(1-p_{1}\right)+p_{1} \frac{\Lambda_{20}}{\sigma_{20} A_{20}} \\
& =\sigma_{1}\left(1-p_{1}\right)+p_{1} \zeta^{2} \sigma_{20}^{f_{2}-1} \\
\sigma_{20} & =\sigma_{2}\left(1-p_{2}\right)+p_{2} \frac{\Lambda_{10}}{\sigma_{10} A_{10}} \\
& =\sigma_{2}\left(1-p_{2}\right)+p_{2} \zeta^{1} \sigma_{10}^{f_{1}-1} .
\end{aligned}
$$

Conversions of end-groups $p_{1}$ and $p_{2}$ are convenient variables for writing the solution of eqs (114)-(123) in a way which is independent from changes of volume and of apparent rate constant $k_{12}$ with end-group concentrations, and thus with time. The following relations are independent of volume changes:

$$
\begin{gathered}
A_{1}=f_{1} U^{1}\left(1-p_{1}\right) \\
A_{2}=f_{2} U^{2}\left(1-p_{2}\right) \\
L_{12}=L_{21}=f_{1} U^{1} p_{1}=f_{2} U^{2} p_{2} .
\end{gathered}
$$

Both conversions are related through the initial stoichiometric ratio $r$ :

$$
p_{2}=\frac{p_{1}}{r}=\frac{f_{1} U^{1}}{f_{2} U^{2}} p_{1} .
$$

Solution of eqs (114)-(127) leads to:

$$
\frac{\Lambda_{1}}{\sigma_{1} A_{1}}=\frac{\Lambda_{10}}{\sigma_{10} A_{10}}=\zeta^{1} \sigma_{10}^{f_{1}-1}
$$

A double root $\sigma_{10}=\sigma_{20}=1$, when $\sigma_{1}=\sigma_{2}=$ $\zeta^{1}=\zeta^{2}=1$, defines the gel point, which occurs when

$$
p_{1} p_{2}=\frac{1}{\left(f_{1}-1\right)\left(f_{2}-1\right)} .
$$

The physically meaningful roots of eqs (123) and (124) when $\sigma_{1}=\sigma_{2}=\zeta^{1}=\zeta^{2}=1$ are the lowest real positive roots not greater than 1 . When at least one of the final values of variables $\sigma_{1}, \sigma_{2}, \zeta^{1}$ or $\zeta^{2}$ is not 1 , the roots must be found by a continuation procedure.

Extinction probabilities can be computed as follows:

$$
\begin{aligned}
v_{12} & =\frac{\bar{X}_{12}(1,1)}{L_{12}}=\left.\frac{\Lambda_{20}}{\sigma_{20} A_{20}}\right|_{\sigma_{1}=\sigma_{2}=1} \\
& =\left.\sigma_{20}^{f_{2}-1}\right|_{\sigma_{1}}=\sigma_{2}=1 \\
v_{21} & =\frac{\bar{X}_{21}(1,1)}{L_{21}}=\left.\frac{\Lambda_{10}}{\sigma_{10} A_{10}}\right|_{\sigma_{1}=\sigma_{2}=1} \\
& =\left.\sigma_{10}^{f_{1}-1}\right|_{\sigma_{1}}=\sigma_{2}=1 .
\end{aligned}
$$

The concentrations of EANJ, EANC and other gel properties related to elastic praperties are obtained by computing the distributions $\bar{G}^{1}$ and $\bar{G}^{2}$ of numbers of end-groups and linking groups around each repeating unit, leading to mass balances

$$
\begin{aligned}
k_{12}^{-1} \frac{\partial \bar{G}^{1}}{\partial t}= & \frac{\partial \bar{G}^{1}}{\partial \log \alpha_{1}} A_{2}\left(\frac{\zeta_{12}}{\alpha_{1}}-1\right) \\
& +\frac{\partial \bar{G}^{1}}{\partial \log \alpha_{2}} A_{1}\left(\frac{\zeta_{21}}{\alpha_{2}}-1\right) \\
k_{12}^{-1} \frac{\partial \bar{G}^{2}}{\partial t}= & \frac{\partial \bar{G}^{2}}{\partial \log \alpha_{1}} A_{2}\left(\frac{\zeta_{12}}{\alpha_{1}}-1\right)
\end{aligned}
$$




$$
\begin{aligned}
& \quad+\frac{\partial \bar{G}^{2}}{\partial \log \alpha_{2}} A_{1}\left(\frac{\zeta_{21}}{\alpha_{2}}-1\right) \\
\left.\bar{G}^{1}\right|_{t=0}= & U^{1} \alpha_{10}^{f_{1}} \\
\left.\bar{G}^{2}\right|_{t=0}= & U^{2} \alpha_{20}^{f_{2}} .
\end{aligned}
$$

The characteristics of eqs (138) and (139) are the same, being determined by the system of ordinary differential equations

$$
\begin{aligned}
& \frac{\mathrm{d} \log \alpha_{1}}{\mathrm{~d} t} / k_{12}=A_{2}\left(\frac{\zeta_{12}}{\alpha_{1}}-1\right) \\
& \frac{\mathrm{d} \log \alpha_{2}}{\mathrm{~d} t} / k_{12}=A_{1}\left(\frac{\zeta_{21}}{\alpha_{2}}-1\right)
\end{aligned}
$$

which are easily solved, leading to

$$
\begin{aligned}
\alpha_{10} & =\zeta_{12}+\left(1-p_{1}\right)\left(\alpha_{1}-\zeta_{12}\right) \\
\alpha_{20} & =\zeta_{21}+\left(1-p_{2}\right)\left(\alpha_{2}-\zeta_{21}\right) \\
\bar{G}^{1}(\alpha, \zeta) & =U^{1}\left[p_{1} \zeta_{12}+\left(1-p_{1}\right) \alpha_{1}\right]^{f_{1}} \\
\bar{G}^{2}(\alpha, \zeta) & =U^{2}\left[p_{2} \zeta_{21}+\left(1-p_{2}\right) \alpha_{2}\right]^{f_{2}} .
\end{aligned}
$$

These solutions were not unexpected. They mean that repeating units $\mathbf{U}^{1}$ are surrounded by $\boldsymbol{A}_{1}$ end-groups or $\mathrm{L}_{12}$ linking groups, their numbers following a binomial distribution, their total number being $f_{1}$. An analogous result is valid for the units $\mathbf{U}^{2}$ :

$$
\begin{aligned}
& G^{1}=U^{1} p_{1}^{f_{1}-a_{1}}\left(1-p_{1}\right)^{a_{1}} \\
& G^{2}=U^{2} p_{2}^{f_{2}-a_{2}}\left(1-p_{2}\right)^{a_{2}} .
\end{aligned}
$$

The transforms of concentrations of repeating units linked to prescribed numbers of end-groups and $x$ network chains can be computed according to eq. (61) from the knowledge of $\bar{G}^{1}, \bar{G}^{2}$ and the extinction probabilities:

$$
\begin{aligned}
\bar{T}^{1}(\alpha, \zeta)= & U^{1}\left\{p_{1}\left[v_{12}+\xi\left(1-v_{12}\right)\right]\right. \\
& \left.+\left(1-p_{1}\right) \alpha_{1}\right\}^{f_{1}} \\
\bar{T}^{2}(\alpha, \xi)= & U^{2}\left\{p_{2}\left[v_{21}+\xi\left(1-v_{21}\right)\right]\right. \\
& \left.+\left(1-p_{2}\right) \alpha_{2}\right\}^{f_{2}} .
\end{aligned}
$$

Inversion of these transforms leads to the distributions according to the numbers of end-groups and network chains, which are multinomial distributions.

The concentrations of EANJ and EANC are easily computed using eqs (64) and (65) after obtaining the moments $\Gamma_{m n}^{M i j k l} \ldots$ by differentiating eqs (146) and (147), or, preferably, by using directly definitions (62) and (63):

$$
\begin{aligned}
\mu_{e}= & U^{1}\left[1-\phi_{1}^{f_{1}}-p_{1} f_{1}\left(1-v_{12}\right) \phi_{1}^{f_{1}-1}\right. \\
& \left.-\frac{1}{2} p_{1}^{2} f_{1}\left(f_{1}-1\right)\left(1-v_{12}\right)^{2} \phi_{1}^{f_{1}-2}\right] \\
& +U^{2}\left[1-\phi_{2}^{f_{2}}-p_{2} f_{2}\left(1-v_{21}\right) \phi_{2}^{f_{2}-1}\right. \\
& \left.-\frac{1}{2} p_{2}^{2} f_{2}\left(f_{2}-1\right)\left(1-v_{21}\right)^{2} \phi_{2}^{f_{2}-2}\right] \\
v_{e}= & \frac{1}{2} U^{1}\left[p_{1} f_{1}\left(1-v_{12}\right)\left(1-\phi_{1}^{f_{1}-1}\right)\right. \\
& \left.-p_{1}^{2} f_{1}\left(f_{1}-1\right)\left(1-v_{12}\right)^{2} \phi_{1}^{f_{1}-2}\right] \\
& +\frac{1}{2} U^{2}\left[p_{2} f_{2}\left(1-v_{21}\right)\left(1-\phi_{2}^{f_{2}-1}\right)\right.
\end{aligned}
$$

$$
\left.-p_{2}^{2} f_{2}\left(f_{2}-1\right)\left(1-v_{21}\right)^{2} \phi_{2}^{f_{2}-2}\right] .
$$

$\phi_{1}$ and $\phi_{2}$ are the fractions of initial end-groups not leading to network chains:

$$
\begin{aligned}
& \phi_{1}=1-p_{1}+p_{1} v_{12} \\
& \phi_{2}=1,-p_{2}+p_{2} v_{21} .
\end{aligned}
$$

Prediction of the weight fraction of pendant material $w_{p}$ requires the simultaneous knowledge of the weight fraction of sol, $w_{s}$. This can be done using eq. (9) and evaluating, by integration along the characteristics, the moments with respect to numbers of repeating units $\lambda^{1}$ and $\lambda^{2}$ and with respect to numbers of endgroups $\lambda_{1}$ and $\lambda_{2}$ :

$$
\begin{aligned}
w_{s}= & \bar{M}_{n}^{1} \lambda^{1}+M_{1} \lambda_{1}+\bar{M}_{n}^{2} \lambda^{2}+M_{2} \lambda_{2} \\
= & \left.\bar{M}_{n}^{1} U^{1} \sigma_{10}^{f_{1}}\right|_{\sigma_{1}=\sigma_{2}=1}+\left.M_{1} A_{1} \sigma_{10}^{f_{1}-1}\right|_{\sigma_{1}=\sigma_{2}=1} \\
& +\left.\bar{M}_{n}^{2} U^{2} \sigma_{10}^{f_{1}}\right|_{\sigma_{1}=\sigma_{2}=1}+\left.M_{2} A_{2} \sigma_{20}^{f_{2}-1}\right|_{\sigma_{1}=\sigma_{2}=1} \\
= & U^{1} v_{21}\left[\bar{M}_{n}^{1} \phi_{1}+M_{1} f_{1}\left(1-p_{1}\right)\right] \\
& +U^{2} v_{12}\left[\bar{M}_{n}^{2} \phi_{2}+M_{2} f_{2}\left(1-p_{2}\right)\right] .
\end{aligned}
$$

Use of eq. (64) yields the following expression, allowing $w_{P}$ to be computed:

$$
\begin{aligned}
w_{s}+w_{P}= & U^{1}\left\{\overline { M } _ { n } ^ { 1 } \left[\phi_{1}^{f_{1}}+p_{1} f_{1}\left(1-v_{12}\right) \phi_{1}^{f_{1}-1}\right.\right. \\
& \left.+\frac{1}{2} p_{1}^{2} f_{1}\left(f_{1}-1\right)\left(1-v_{12}\right)^{2} \phi_{1}^{f_{1}-2}\right] \\
& +M_{1} f_{1}\left(1-p_{1}\right)\left[\phi_{1}^{f_{1}-1}+p_{1}\left(1-v_{12}\right)\right. \\
& \times\left(f_{1}-1\right) \phi_{1}^{f_{1}-2}+\frac{1}{2} p_{1}^{2}\left(f_{1}-1\right) \\
& \left.\left.\times\left(f_{1}-2\right)\left(1-v_{12}\right)^{2} \phi_{1}^{f_{1}-3}\right]\right\} \\
& +U^{2}\left\{\overline { M } _ { n } ^ { 2 } \left[\phi_{2}^{f_{2}}+p_{2} f_{2}\left(1-v_{21}\right) \phi_{2}^{f_{2}-1}\right.\right. \\
& \left.+\frac{1}{2} p_{2}^{2} f_{2}\left(f_{2}-1\right)\left(1-v_{21}\right)^{2} \phi_{2}^{f_{2}-2}\right] \\
& +M_{2} f_{2}\left(1-p_{2}\right)\left[\phi_{2}^{f_{2}-1}+p_{2}\left(1-v_{21}\right)\right. \\
& \times\left(f_{2}-1\right) \phi_{2}^{f_{2}-2}+\frac{1}{2} p_{2}^{2}\left(f_{2}-1\right) \\
& \left.\left.\times\left(f_{2}-2\right)\left(1-v_{21}\right)^{2} \phi_{2}^{f_{2}-3}\right]\right\} .
\end{aligned}
$$

Langley's trapping factor is computed similarly, starting from eq. (65) and taking into account that $T_{\text {emAX }}^{*}=1$, which occurs at full conversion of both end-groups, possible only at stoichiometric balance $r=1$ :

$$
\begin{aligned}
\sqrt{T_{e}}= & 1-U^{1}\left\{\bar{M}_{n}^{1}\left[\phi_{1}^{f_{1}}+p_{1} f_{1}\left(1-v_{12}\right) \phi_{1}^{f_{1}-1}\right]\right. \\
& +M_{1} f_{1}\left(1-p_{1}\right)\left[\phi_{1}^{f_{1}-1}+p_{1}\left(1-v_{12}\right)\right. \\
& \left.\left.\times\left(f_{1}-1\right) \phi_{1}^{f_{1}-2}\right]\right\} \\
& -U^{2}\left\{\bar{M}_{n}^{2}\left[\phi_{2}^{f_{2}}+p_{2} f_{2}\left(1-v_{21}\right) \phi_{2}^{f_{2}-1}\right]\right. \\
& +M_{2} f_{2}\left(1-p_{2}\right)\left[\phi_{2}^{f_{2}-1}+p_{2}\left(1-v_{21}\right)\right. \\
& \left.\left.\times\left(f_{2}-1\right) \phi_{2}^{f_{2}-2}\right]\right\} .
\end{aligned}
$$

These results are identical to earlier predictions using TBP, as it will be shown next. They were quite useful to check the results of the computer program later used for analysing the curing of epoxy resins. 


\section{COMPARISON WITH PREDICTIONS OF THEORY OF BRANCHING PROCESSES}

Theory of branching processes (TBP), as introduced by Good and Gordon in polymer science (Good, 1962; Gordon, 1962; Gordon and Scantlebury, 1964), defines two probability generating functions for each repeating unit $U^{M}$, named $F_{0}^{M}(\zeta)$ and $F_{1}^{M}(\zeta)$. The former is the vectorial discrete transform of the normalized distribution of numbers of repeating units $\mathrm{U}^{1} \ldots \mathrm{U}^{\mathrm{N}}$ linked to a repeating unit $\mathrm{U}^{\mathrm{M}}$ in the root of a tree. If the repeating unit $\mathrm{U}^{\mathrm{M}}$ is not at the root of a tree, then $F_{1}^{M}(\zeta)$ is taken as the vectorial transform of the normalized distribution of the numbers of repeating units $U^{1} \ldots U^{N}$ linked to that unit, independently of its position in the tree (this independence is not guaranteed outside the chemical equilibrium, and may invalidate TBP in that case). These two distributions are related through

$$
F_{1}^{M}(\zeta)=\frac{\sum_{n=1}^{N} \frac{\partial F_{0}^{M}}{\partial \zeta^{n}}}{\sum_{n=1}^{N} \frac{\partial F_{0}^{M}}{\partial \zeta^{n}}\left(1^{N}\right)} .
$$

For instance, in the polycondensation of $\mathbf{U}^{1}\left(\mathbf{A}_{1}\right)_{r_{1}}$ and $\mathrm{U}^{2}\left(\mathrm{~A}_{2}\right)_{\mathrm{r}_{2}}$,

$$
\begin{aligned}
& F_{0}^{1}=\left(1-p_{1}+p_{1} \zeta^{2}\right)^{f_{1}} \\
& F_{1}^{1}=\left(1-p_{1}+p_{1} \zeta^{2}\right)^{f_{1}-1} \\
& F_{0}^{2}=\left(1-p_{2}+p_{2} \zeta^{1}\right)^{f_{2}} \\
& F_{1}^{2}=\left(1-p_{2}+p_{2} \zeta^{1}\right)^{f_{2}-1} .
\end{aligned}
$$

According to TBP, the discrete transform $\bar{W}^{M}(\zeta)$ of the distribution with respect to numbers of repeating units of trees with a repeating unit $\mathbf{U}^{\mathrm{M}}$ in their root is given by

$$
\bar{W}^{M}(\zeta)=\zeta^{M} F_{0}^{M}(u)
$$

in which vector $v$ verifies the system of algebraic equations

$$
u_{i}=\zeta^{i} F_{1}^{i}(v) \quad(i=1, N) .
$$

These results can be compared with the kinetic approach taking into account the relation between $\vec{P}$ and $\bar{W}^{M}$ :

$\bar{W}^{M}(\zeta)=\frac{\partial \bar{P}}{\partial \log \zeta^{M}}\left(\mathbf{1}_{G}, \zeta\right) / U^{M}=\Lambda^{M}\left(\mathbf{1}_{G}, \zeta\right) / U^{M}$.

In the example of the alternating polycondensation of two monomers,

$$
\begin{aligned}
v_{1} & =\zeta^{1}\left(1-p_{1}+p_{1} v_{2}\right)^{f_{1}-1} \\
v_{2} & =\zeta^{2}\left(1-p_{2}+p_{2} v_{1}\right)^{f_{2}-1} \\
\bar{W}^{1} & =\zeta^{1}\left(1-p_{1}+p_{1} v_{2}\right)^{f_{1}} \\
\bar{W}^{2} & =\zeta^{2}\left(1-p_{2}+p_{2} v_{1}\right)^{f_{2}} .
\end{aligned}
$$

The mathematical equivalence with results of kinetic approach is readily proved, since

$$
\begin{aligned}
& \left.\sigma_{10}\right|_{\sigma_{1}=\sigma_{2}=1}=1-p_{1}+p_{1} v_{2} \\
& \left.\sigma_{20}\right|_{\sigma_{1}}=\sigma_{2}=1=1-p_{2}+p_{2} v_{1} \text {. }
\end{aligned}
$$

Prediction of concentrations of EANJ and EANC or other gel properties through TBP is based on the calculation of extinction probabilities $u^{M}$ of each repeating unit (not of each linking group). They are defined as the probabilities of a root starting by a certain repeating unit $\mathrm{U}^{\mathbf{M}}$ being finite, and so they verify the algebraic equations

$$
u^{M}=F_{1}^{M}(\mathbf{u}) .
$$

So, in the case of the alternating polycondensation of two monomers,

$$
\begin{aligned}
& u^{1}=\left(1-p_{1}+p_{1} u^{2}\right)^{f_{1}-1} \\
& u^{2}=\left(1-p_{2}+p_{2} u^{1}\right)^{f_{2}-1} .
\end{aligned}
$$

It is immediately seen that $u^{1}$ and $u^{2}$ are equal to the non-zero components of the matrix of extinction probabilities previously computed by the kinetic method through eqs (136) and (137), taking into account the equivalence relations (171) and (172):

$$
\begin{gathered}
u^{1}=v_{21} \\
u^{2}=v_{12} .
\end{gathered}
$$

A relation similar to eq. (61) can generally be written for each repeating unit:

$$
\bar{T}^{M}\left(\mathbf{1}_{G}, \xi\right)=U^{M} F_{0}^{M}\left[\mathbf{u}+\xi\left(\mathbf{1}^{N}-\mathbf{u}\right)\right] .
$$

Note that in classical TBP there is no systematic way of taking into account the pendant end-groups in each repeating unit, so as to add their mass when molecular weights or weight fractions are needed. However, in the alternating polycondensation discussed here, one would write eqs (146) and (147) for $\bar{G}^{1}$ and $\bar{G}^{2}$ using a probabilistic reasoning, and then eqs (156)-(158) for predicting weight fraction of sol, weight fraction of pendant material and Langley's factor would follow. Thus, there is an exact mathematical equivalence between the kinetic approach and the probabilistic description based on TBP, in the case of this alternating polycondensation.

It is also possible to use TBP for predicting gel structure with monomers which may have more than one elastically active junction. In that case, it is necessary to introduce the vectorial discrete transforms of the normalized distributions of numbers of repeating units $\mathrm{U}^{1} \ldots \mathrm{U}^{\mathrm{N}}$ directly linked to each junction $\mathrm{G}^{\mathrm{MJ}}$, which we will name $F_{0}^{M J}(\zeta)$.

The probability that a tree with a junction $\mathrm{G}^{\mathrm{MJ}}$ at its root does not lead to infinity is $F_{0}^{M J}(u)$. So, the generalization of eq. (178) is

$$
\begin{aligned}
\bar{T}^{M J}\left(\mathbf{1}_{G}, \xi\right)= & U^{M} \rho^{M J} \prod_{K=1}^{J^{M}}\left[F_{0}^{M K}(\mathbf{u})(1-\xi)+\xi\right]^{g^{M / K}} \\
& \times F_{0}^{M J}\left[\mathbf{u}+\xi\left(1^{N}-\mathbf{u}\right)\right] .
\end{aligned}
$$

If needed, it is possible to predict the dependence of the numbers of end-groups by a probabilistic reasoning and to write expressions for the $\bar{T}^{M J}(\alpha, \xi)$.

\section{NUMERICAL CASE STUDIES}

The practical performance of the mathematical treatment just described was checked by simulating 
the cure of a tetraepoxide with a primary diamine, a system of practical importance in the field of composite materials. The same simplified kinetic scheme used by Tsou and Peppas (1988) was chosen, so that the results could be compared with an earlier treatment by TBP.

We took the ratios of kinetic constants of epoxy reaction with secondary and primary amines $k_{S} / k_{P}=0.2$ (a value of 0.5 would correspond to equal reactivity of each hydrogen) and of etherification to addition to primary amines $k_{E} / k_{P}=0.055$, giving a certain character of polyaddition (See Table 3). Molecular weights of monomers were taken as the corresponding values of 4,4'-diaminodiphenylsulphone and tetraglycidyl-4,4'-diaminodiphenylmethane, $M^{1}=248$ and $M^{2}=422$, respectively. $M_{i}=0$ for all $i=1,5$, since there is no liberation of by-products.

In Fig. 1, the evolution of weight fraction of sol as a function of epoxide conversion is shown, and in Fig. 2 weight-average molecular weight of sol vs epoxide conversion are shown. In both figures, three different values of the initial molar ratio of active hydrogens in amines to epoxide group $r$ were used $(r=1$ is the value for stoichiometric balance in the absence of etherification).

Newton iteration for computing the curves after gelation converged without problems, except at the end of the reaction, when the concentration of pri-

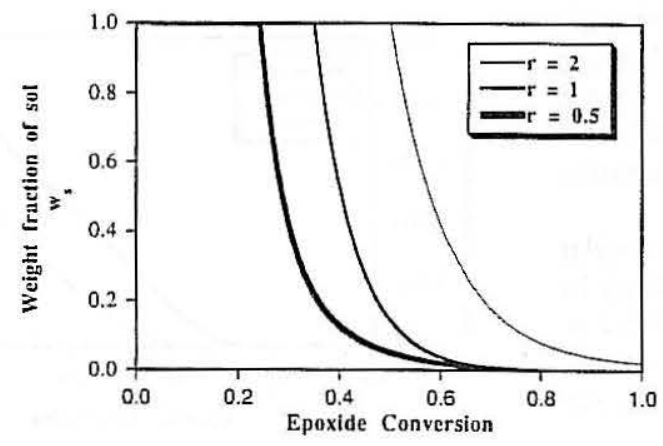

Fig. 1. Weight fraction of sol $w_{s}$ vs epoxide conversion in the curing of a tetraepoxide by a diamine (kinetic parameters in Table 3) for three different values of initial molar ratio of active hydrogens in amines and to epoxide groups $r$.

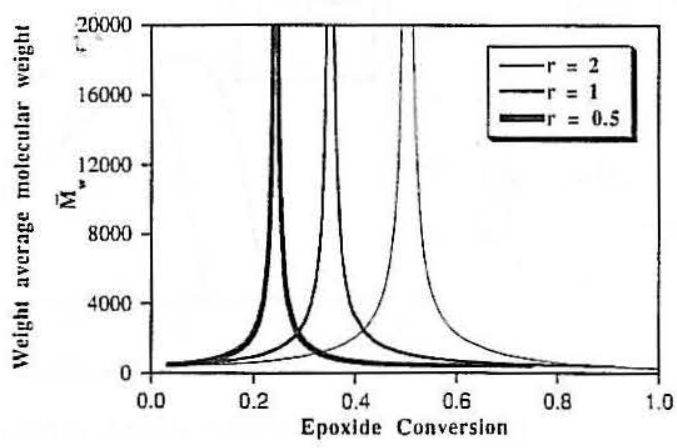

Fig. 2. Weight-average molecular weight of sol $\bar{M}_{w}$ vs epoxide conversion, for three different values of initial molar ratio of active hydrogens in amines to epoxide groups $r$. mary amines becomes virtually nil and this brings about some numerical problems due to round-off.

Results by TBP are shown for comparison purposes in Figs 3 and 4. There are some slight differences relatively to the more correct results of the kinetic treatment in the presence of the etherification reaction, mostly as far as post-gel molecular weights are concerned. It would be difficult to detect these differences experimentally. In the absence of the etherification reaction, the results would be exactly the same for this chemical system [for a further discussion of the differences between the two approaches, see the recent paper by Sarmoria and Miller (1991)].

Of course, TBP breaks down for irreversible polyadditions: it predicts that MWD for a polyaddition without termination is a Schulz-Flory distribution instead of a Poisson's distribution.

Some care is required in order to use our approach for predicting gel structure and its elastic properties, because the monomers contain reasonably long flexible chains and so are not point-like. The diamine monomer $\mathrm{U}^{1}$ contains $\rho^{11}=2$ junctions $\mathrm{G}^{11}$ of a single kind, which may be identified with the nitrogen atoms. Each of them is initially attached to $f_{1}^{11}=1$ primary amine groups $\mathbf{A}_{1}$ and to no other reactive groups $\left(f_{2}^{11}=\cdots=f_{5}^{11}=0\right)$. The two junctions are linked by an inert chain $\mathrm{L}^{111}$, which can become elastically active, and therefore $g^{111}=1$ (Fig. 5).

The $\mathrm{N}, \mathrm{N}^{\prime}, \mathrm{N}^{\prime \prime}, \mathrm{N}^{\prime \prime \prime}$ tetraglycidyl monomer $\mathrm{U}^{2}$ is supposed to contain $\rho^{21}=2$ junctions $\mathrm{G}^{21}$ of a single

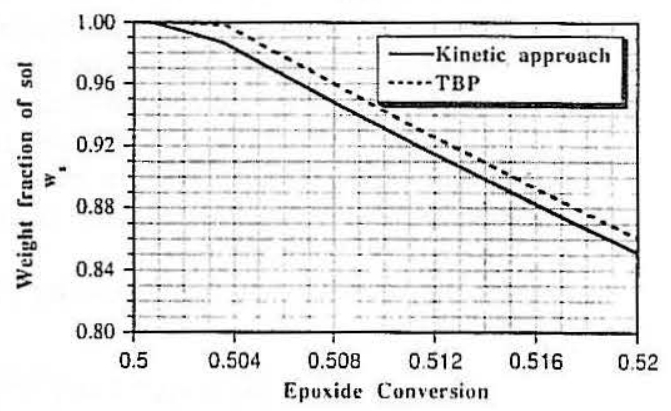

Fig. 3. Comparison between weight fraction of $\mathrm{sol}_{\mathrm{s}}$ as predicted by kinetic approach and by TBP, for the curing of a tetraepoxide with a diamine.

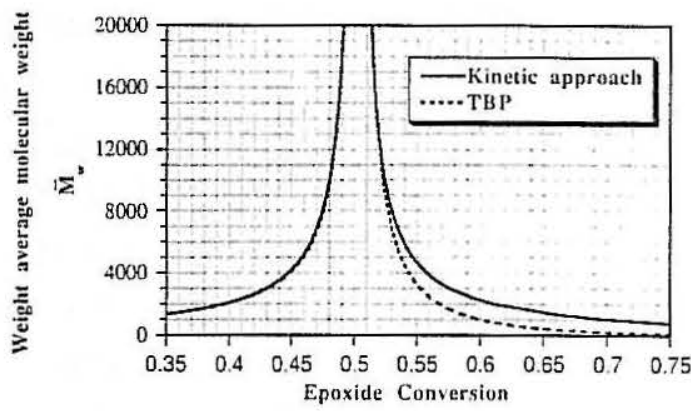

Fig. 4. Comparison between weight-average molecular weight of sol $\bar{M}_{w}$ as predicted by kinetic approach and by TBP, for the curing of a tetraepoxide with a diamine. 


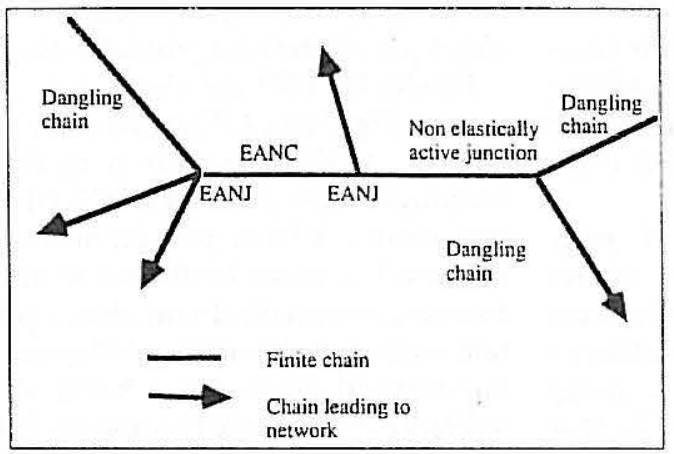

Fig. 5. Elastically active junctions and chains.

kind, which may be identified with the nitrogen atoms. Each of them is initially attached to two epoxide groups $\mathbf{A}_{2}$, and so $f_{2}^{21}=2$ and to no other reactive groups $\left(f_{1}^{21}=f_{3}^{21}=\cdots=f_{5}^{21}=0\right)$. The two junctions are linked by an inert chain $\mathbf{L}^{211}$, which can become elastically active, and therefore $g^{211}=1$.

Tsou and Peppas (1988) presented a slightly different model of the elastic behavior of the tetraepoxide repeating unit. The nitrogen atoms and the two $-\mathrm{CH}-$ groups attached to

$$
{ }_{\mathrm{O}}^{-} \mathrm{CH}_{2}
$$

were considered to be independent junctions. Each of them can lead to at most three network chains, when the epoxide group reacts with amines or hydroxyls and the resulting hydroxyl group is also etherified. However, the two pairs of chains of methylene groups between nitrogens and - $\mathrm{CH}-$ groups are too short for being elastically active. Therefore, their concentration must be subtracted from the overall EANC.

It follows from these structures that molecular weights of each monomer can be evenly shared by each of their two junctions, and so $M^{11}=248 / 2=$ 124 and $M^{21}=422 / 2=211$.

The corresponding predictions by TBP were computed using

$$
\begin{aligned}
& F_{0}^{1}=\left[a_{p}+a_{s} \zeta^{2}+a_{t}\left(\zeta^{2}\right)^{2}\right]^{2}(180) \\
& F_{0}^{2}=\left[e_{0}+e_{a} \zeta^{1}+e_{e} \zeta^{2}+e_{e} \zeta^{1} \zeta^{2}+e_{e e}\left(\zeta^{2}\right)^{2}\right]^{4} F_{0}^{11}=a_{p}+a_{s} \zeta^{2}+a_{t}\left(\zeta^{2}\right)^{2} \\
& F_{0}^{21}=\left[e_{0}+e_{a} \zeta^{1}+e_{e} \zeta^{2}+e_{e} \zeta^{1} \zeta^{2}+e_{e e}\left(\zeta^{2}\right)^{2}\right]^{2}(183) \\
& \bar{T}^{11}\left(1_{G}, \xi\right)= 2 U^{1}\left\{\left[a_{p}+a_{s} u^{2}+a_{t}\left(u^{2}\right)^{2}\right](1-\xi)+\zeta\right\} \\
& \times\left\{a_{p}+a_{s}\left[u^{2}+\xi\left(1-u^{2}\right)\right]\right. \\
&\left.+a_{t}\left[u^{2}+\xi\left(1-u^{2}\right)\right]^{2}\right\} \\
& \bar{T}^{21}\left(1_{G}, \xi\right)= 2 U^{2}\left\{\left[e_{0}+e_{a} u^{1}+e_{e} u^{2}+e_{e} u u^{1} u^{2}\right.\right. \\
&\left.\left.+e_{e e}\left(u^{2}\right)^{2}\right]^{2}(1-\xi) \times \xi\right\} \\
& \times\left\{e_{0}+e_{a}\left[u^{1}+\xi\left(1-u^{1}\right)\right]\right. \\
&+e_{e}\left[u^{2}+\xi\left(1-u^{2}\right)\right] \\
&+e_{e}\left[u^{1}+\xi\left(1-u^{1}\right)\right]\left[u^{2}+\xi\left(1-u^{2}\right)\right] \\
&\left.+e_{e e}\left[u^{2}+\xi\left(1-u^{2}\right)\right]^{2}\right\}^{2} .
\end{aligned}
$$

In these expressions, variables $a_{p}, a_{s}$ and $a_{t}$ are, respectively, concentrations of primary, secondary and tertiary amines, normalized by initial primary amine concentration. $e_{0}, e_{a}, e_{\mathrm{c}}$ and $e_{e e}$ are, respectively, concentrations of unreacted epoxides, epoxides which have reacted with an amine and epoxides which have reacted with one and two epoxide groups, normalized by initial epoxide concentration. They are computed as functions of primary amine conversion according to Tsou and Peppas (1988).

In Figs 6-8, the predicted changes of concentrations of EANJ, EANC and weight fraction of pendant materials vs epoxide conversion are shown.

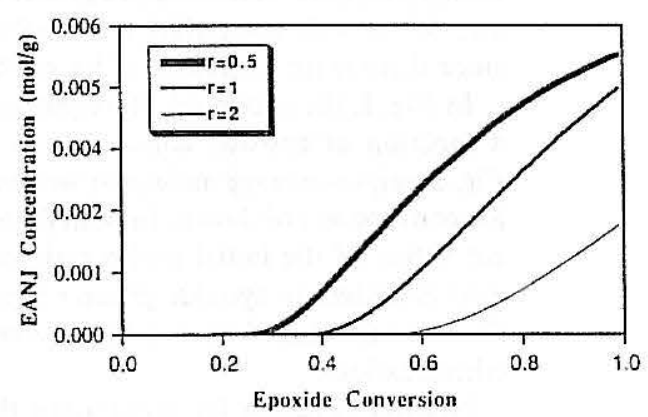

Fig. 6. EANJ vs epoxide conversion for three different values of molar ratio of active hydrogens in amines to epoxide groups $r$, for the curing of a tetraepoxide with a diamine.

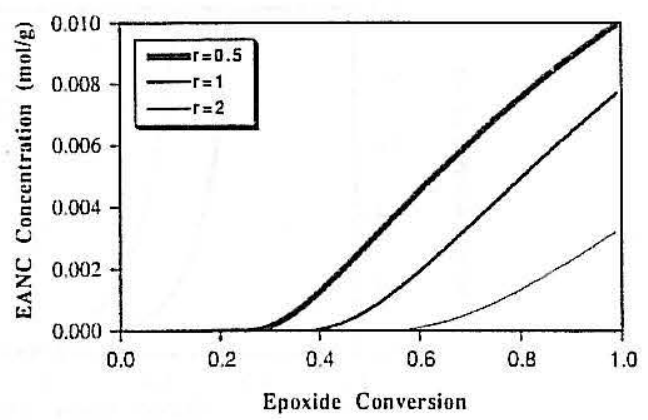

Fig. 7. EANC vs epoxide conversion for three different values of molar ratio of active hydrogens in amines to epoxide groups $r$, for the curing of a tetraepoxide with a diamine.

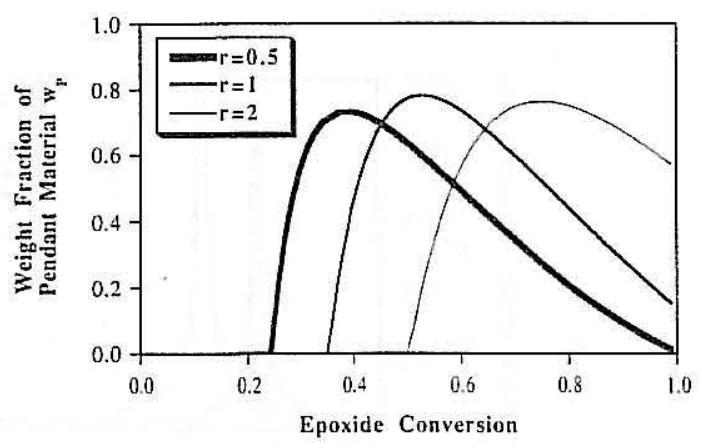

Fig. 8. Weight fraction of pendant material vs epoxide conversion for three different values of molar ratio of active hydrogens in amines to epoxide groups $r$, for the curing of a tetraepoxide with a diamine. 


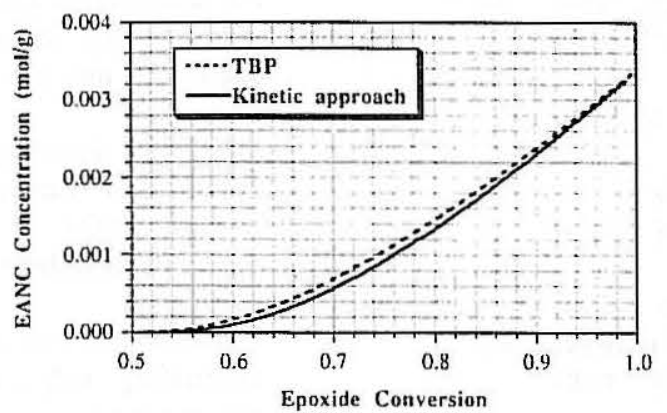

Fig. 9. Comparison between EANC as predicted by kinetic approach and by TBP, for the curing of a tetraepoxide with a diamine $(r=2)$.

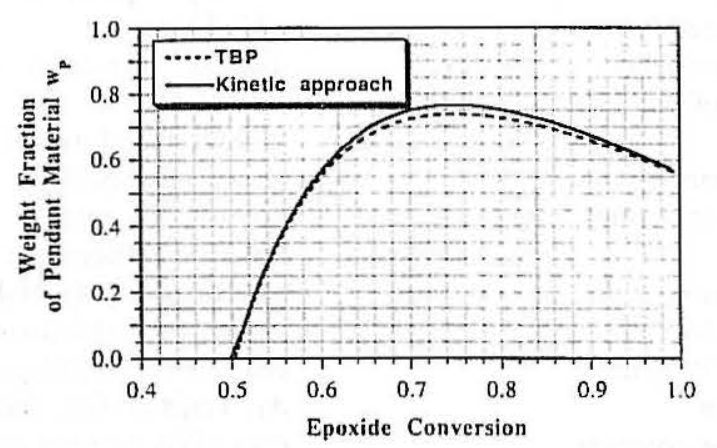

Fig. 10. Comparison between weight fraction of pendant material as predicted by kinetic approach and by TBP, for the curing of a tetraepoxide with a diamine $(r=2)$.

A comparison with results obtained through the use of TBP and of kinetic approach is shown in Figs 9 and 10. Numerical differences between the predictions of the kinetic approach and TBP are small for this chemical system, as for the weight-average molecular weight and fraction of sol. It would be necessary to have a much greater relative importance of the polyetherification of epoxides with hydroxyls to find larger errors in TBP predictions.

\section{CONCLUSIONS}

This work has removed the main drawbacks preventing the kinetic approach to have a more widespread use in polymer science and engineering when dealing with non-linear polymerizations: prediction of gel properties became possible and the whole treatment is free from the need of an analytical solution to the mass balance equations of polymer species and pseudo-species, making possible the analysis of complex chemical systems.

A radically different description of molecular structure is needed in order to compute properties of sol, such as average gyration radius and light-scattering properties, which can only be done, for the moment, using TBP (Burchard, 1982). The same problem arises in the modelling of reversible copolymerization systems.

Computations taking into account loop formation (a limited number of small loops) still need to be tried.

It cannot be assessed for the moment whether the increase in complexity coming from the expected large number of pseudo-repeating units and reactive groups can lead to reasonable computing times, and nothing is known from the comparative performance of Monte-Carlo methods.

Also, some automatic means of defining molecular fragments and computing stoichiometric coefficients taking account of intramolecular reactions seems important, in order to allow a realistic modelling of most polycondensation reactions and non-linear radical polymerizations.

There still remains a huge amount of work to be done in the modelling of complex radical polymerizations using the approach just described, although no insurmountable obstacles are likely to exist.

Acknowledgments-Financial support by JNICT (project $502 / 90 / \mathrm{MPF}$ ) is gratefully acknowledged, as well as earlier contributions by undergraduate students João C. F. Fernandes and António M. L. Ramos.

\section{a \\ $a_{i}$}

$a_{s}$

$a_{t}$

$A_{\mathrm{i}}$

$e_{0}$

$e_{a}$

$e_{e}$

$f_{i}^{m}$

$f_{i}^{M J}$

$F\left(t, \sigma_{1}, \ldots, \sigma_{G}\right.$

$\left.\bar{P}, \Lambda_{1}, \ldots, \Lambda_{G}\right)$

$F_{0}^{M}(\zeta)$

\section{NOTATION}

vector of numbers of end-groups number of end-groups $\mathbf{A}_{\mathbf{i}}$ mole concentration of primary amines, normalized by initial primary amine concentration mole concentration of secondary amines, normalized by initial primary amine concentration mole concentration of tertiary amines, normalized by initial primary amine concentration mole concentration of end-groups $\mathbf{A}_{\mathbf{i}}(i=1, G)$ mole concentration of unreacted epoxides, normalized by initial epoxide concentration

mole concentration of epoxides which have reacted with one amine, normalized by initial epoxide concentration

mole concentration of epoxides which have reacted with one epoxide, normalized by initial epoxide concentration

mole concentration of epoxides which have reacted with two epoxide groups, normalized by initial epoxide concentration number of end-groups $\mathbf{A}_{\mathbf{i}}$ in monomer $m$

number of end-groups $\boldsymbol{A}_{\mathrm{i}}$ attached to junction $G^{\mathrm{MJ}}$ in monomer $M$ right-hand side of eq. (20)

link probability generating function of repeating units $\mathrm{U}^{\mathrm{M}}$ in the root of a tree 


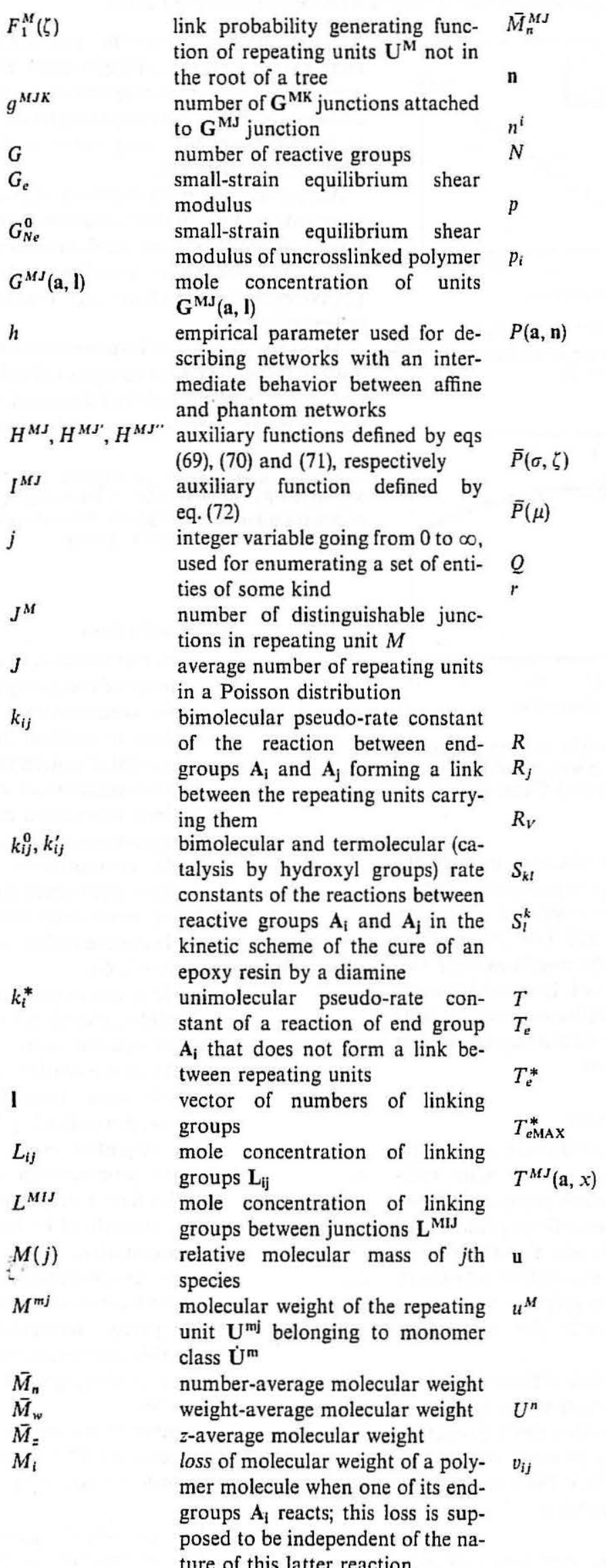

number-average molecular weight allocated to junction $\mathrm{G}^{\mathrm{MJ}}$ vector of numbers of repeating units

number of repeating units $\mathbf{U}^{\mathrm{i}}$ number of different kinds of repeating units parameter in Schulz-Flory's distribution conversion of end-group $A_{i}$ in an alternating polycondensation of two monomers mole concentration of the set of polymer molecules $\mathbf{P}(\mathbf{a}, \mathbf{n})$, containing $n$ repeating units and a end-groups of the several possible kinds

discrete transform of polymer VNMND

discrete transform of the NMWD of polymer species volume flow rate

initial stoichiometric ratio in an alternating polycondensation of two monomers (ratio of initial concentrations of end-groups $\mathbf{A}_{2}$ and $\mathbf{A}_{1}$, respectively), such as in the case of epoxy resin curing ideal gas constant

rate of formation by chemical reaction of $j$ th species

relative rate of change of volume caused by chemical reaction component of the Jacobian of $\sigma_{k}$, as defined by eq. (34)

auxiliary variables used for computing matrix $\left[S_{k l}\right]$, as defined by eq. (35)

absolute temperature

Langley's trapping factor for chain entanglements

nonnormalized Langley's trapping factor

maximum value of nonnormalized Langley's trapping factor

mole concentration of $\mathrm{G}^{\mathrm{MJ}}$ junctions connected to a end-groups and $x$ network chains

vector of the extinction probabilities of repeating units (see below) extinction probability of repeating unit $\mathrm{U}^{\mathrm{M}}$, i.e. probability that a rooted tree beginning by such an unit is finite

mole concentration of repeating units $\mathbf{U}^{n}(n=1, N)$

extinction probability of chains starting in a linking unit $\mathrm{L}_{\mathrm{ij}}$ in the direction going from the repeating unit previously carrying group $A_{\mathrm{i}}$ to the one previously carrying 
end-group $A_{j}$; it is the fraction of those chains which do not go to infinity

$v^{M J K}$

V

$w$

$w_{p}$

$w_{s}$
$W_{i j}$

$W_{i}^{*}$

$\bar{W}^{M}(\zeta)$

$x$

$X_{i j}(\mathrm{a})$

$X^{\mathrm{MIJ}}$ (a)

$Z_{k l}$

$1_{G}, 1^{N}$

$1_{G G}$

Greek letters

$x$

$\alpha_{i}$
$\Gamma_{m n+\ldots}^{M J j h l .}$

$\zeta$

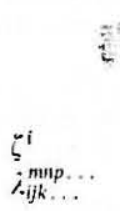

$i_{M_{i}}$

$\Lambda_{i j k \ldots}^{m n p}$ extinction probability of chains starting in a junction $\mathrm{G}^{\mathrm{MJ}}$ towards a junction $\mathrm{G}^{\mathrm{MK}}$; it is the fraction of those chains which do not go to infinity

reaction volume

weight fraction

weight fraction of pendant material

weight fraction of sol

change of volume by reaction of

1 mole of end-groups $A_{i}$ and $A_{j}$

change of volume by reaction of

1 mole of end-group $A_{i}$ by a reaction not forming links

discrete transform of the distribution with respect to numbers of repeating units of trees with a repeating unit $\mathbf{U}^{\mathbf{M}}$ in their root

number of network chains

mole concentration of dangling chains attached to a end-groups, $\mathrm{X}_{\mathrm{ij}}$ (a)

mole concentration of dangling chains attached to a end-groups, $\mathrm{X}^{\mathrm{MII}}(\mathrm{a})$

component of the Jacobian of $\alpha_{k}$, $\left(=\partial \log \alpha_{k} / \partial \log \alpha_{10}\right)$ as defined by eq. (96)

vectors with all their $G$ or $N$ components equal to 1

matrix $G$ by $G$ with all components equal to 1

vector of Laplace parameters of discrete transform of distributions of concentrations of junctions $\mathrm{G}^{\mathrm{MJ}}(\mathrm{a}, \mathrm{l})$ with respect to their numbers of end-groups a

$i$ th component of vector $\alpha$ partial derivative of $\bar{G}^{M J}$ with respect to $\zeta_{i j}, \zeta_{k l}, \ldots$ and $\sigma_{m}, \sigma_{n}, \ldots$ vector of Laplace parameters of the vectorial discrete transform associated with vectors with vector of numbers of repeating units $\mathbf{n}$ or numbers of linking groups 1 $i$ th component of vector $\zeta$ moment of NMND of polymer species of order 1 with respect to the numbers of end-groups $a_{i}, a_{j}, a_{k}, \ldots$ and of repeating units $n^{m}, n^{n}, n^{p} \ldots$

$i$ th order moment of NMWD of polymer species

partial derivative of $\bar{P}$ with respect to $\sigma_{i}, \sigma_{j}, \sigma_{k}, \ldots$ and $\zeta^{m}, \zeta^{n}, \zeta^{p} \ldots$ $\mu$

$\mu_{\mathrm{e}}$

$v_{e}$

$v_{i j k}$

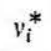

$v_{i j}^{*}$

$\xi$

$\rho^{M J}$

$\tau$

$v_{\mathrm{i}}$

$\phi_{1}, \phi_{2}$

$\omega_{i j}$

$\omega_{i j k}$

$\Omega^{m j}$

\section{Subscripts}

F

$g$

$s$

MAX

0

Superscripts

I, II

$-$

Abbreviations

$A_{\mathbf{i}}$

EANC
Laplace parameter of discrete transform of molecular weight distributions

concentration of elastically effective network junctions (EANJ) concentration of elastically effective network chains (EANC) number of $\mathbf{A}_{\mathbf{k}}$ end-groups formed by reaction of end-group $A_{i}$ with $\mathbf{A}_{\mathrm{j}}$, which are linked to the repeating unit to which $\boldsymbol{A}_{i}$ is attached vector of the $v_{i j}^{*}$, with $j=1, G$ (see below)

number of $\mathbf{A}_{\mathbf{j}}$ end-groups formed by a reaction of end group $A_{i}$ not leading to an intermolecular link Laplace parameter associated with number of network chains $x$ number of $\mathrm{G}^{\mathrm{MJ}}$ junctions in repeating unit $\mathrm{U}^{\mathrm{M}}$

vector of Laplace parameters of the vectorial discrete transform associated with vector a of the numbers of reactive groups of polymer species

$i$ th component of vector $\sigma$

space time (ratio of reactor volume by inlet volume flow rate)

vector of $N$ auxiliary Laplace parameters used in TBP

$i$ th component of the vector of auxiliary Laplace parameters $v$

fractions of initial end-groups $A_{1}$ and $A_{2}$ not leading to network chains

matrix of the $\omega_{i j k}$, with $k=1, G$ (see below)

number of $\boldsymbol{A}_{\mathbf{k}}$ end-groups formed by reaction of end group $\mathbf{A}_{\mathbf{i}}$ and $\mathbf{A}_{\mathbf{j}}$ in both repeating units they are attached to

mole fraction of subspecies $\mathbf{U}^{\mathrm{mj}}$ relative to the overall number of repeating units $\mathbf{U}^{\mathrm{m}}$

feed

gel

sol

upper limit

initial

labels of the alternative sets of apparent rate constants and stoichiometric coefficients discrete transform

reactive group of $i$ th kind elastically active network chain 


\section{EANJ \\ $\mathrm{G}^{\mathrm{MJ}}$ $\mathrm{G}^{\mathrm{MJ}}(\mathrm{a}, \mathrm{l})$ \\ $\mathbf{L}_{\mathrm{ij}}$ \\ $\mathbf{L}^{\text {MJK }}$ \\ MWD \\ NMWD \\ $\mathbf{P}(\mathbf{a}, \mathbf{n})$ \\ TBP \\ $\mathrm{U}^{\mathrm{m}}$ \\ $\mathrm{U}^{\mathrm{mj}}$}

VNMND

$\mathrm{X}_{\mathrm{ij}}(\mathrm{a})$

$\mathrm{X}^{\mathrm{MIJ}}(\mathbf{a})$ elastically active network junction junction in repeating unit $\mathbf{U}^{\mathbf{M}}$ junction $\mathrm{G}^{\mathrm{MJ}}$ which is attached to a end-groups and I linking groups group that links two repeating units, formed by reaction of endgroups $\mathbf{A}_{\mathbf{i}}$ and $\mathbf{A}_{\boldsymbol{j}}$ chain linking $\mathrm{G}^{\mathrm{MIJ}}$ and $\mathrm{G}^{\mathrm{MK}}$ molecular weight distribution number molecular weight distribution

set of polymer molecules with $\mathbf{n}$ repeating units and a end-groups theory of branching processes repeating unit of class $m$ repeating sub-unit of $j$ th kind included in class $\mathbf{U}^{\mathrm{m}}$

vectorial number molecular distribution of numbers of end-groups and repeating units, obtained from function $\mathbf{P}(\mathbf{a}, \mathbf{n})$

dangling chain starting at a linking-group $\mathbf{L}_{i j}$, going towards the repeating unit previously carrying end-group $A_{j}$ and terminated by a end-groups

dangling chain starting at a linking-group between junctions $\mathrm{L}^{\text {MIJ }}$, going towards junction $\mathrm{G}^{\mathrm{MJ}}$ and terminated by a end-groups

\section{REFERENCES}

Argyropoulos, D. S., Berry, R. M. and Bolker, H., 1987a, Polymerization beyond the gel point. I. The molecular weight of sol as a function of the extent of reaction. $J$. Polym. Sci. Polym. Phys. Edn 25, 1191-1202.

Argyropoulos, D. S., Berry, R. M. and Bolker, H., 1987b, Species distribution within the soluble phase beyond the gel point. Macromolecules 20, 357-361.

Argyropoulos, D. S., Berry, R. M. and Bolker, H., 1987c, Polymerization beyond the gel point. 2. A study of the soluble fraction as a function of the extent of reaction. Makromol. Chem. 188, 1985-1992.

Argyropoulos, D. S. and Bolker, H., 1986, Kinetics of gelation in model polycondensates. Ind. Engng Chem. Prod. Res. Dev. 25, 578-582.

Burchard, W., 1982, Static and dynamic light scattering from branched polymers and biopolymers. Adv. Polym. Sci. 48, $1-124$.

Case, L. C., 1960, Branching in polymers. I. Network defects. J. Polym. Sci. 45, 397-404.

Costa, M. R. N. and Villermaux, J., 1988, Mathematical and experimental foundaiions of linear polycondensation modeling. 1. Modeling and simulation of linear, irreversible polycondensation. Ind. Engng Chem. Res. 27, 421-429.

Costa, M. R. N. and Villermaux, J., 1989, Mathematical and experimental foundations of linear polycondensation modeling. 2. Computational aspects of the evaluation of chain length distributions and average degrees of polymerization for linear reversible polycondensation. Ind. Engng Chem. Res. 28, 702-710.

Courant, R. and Hilbert, D., 1962, Methods of Mathematical Physics, Vol. II-Partial Differential Equations, pp. 97-105. Interscience, New York.

Dobson, G. R. and Gordon, M., 1964, Theory of branching processes and statistics of rubber elasticity. J. chem. Phys. 43, 705-713.
Dossin, L. M. and Graessley, W. W., 1979, Rubber elasticity of well-characterized ${ }^{\prime}$ polybutadiene networks. Macromolecules 12, 123.

Dusek, K., 1985, Formation-structure relationships in polymer networks. Brit. Polym. J. 17, 185-189.

Dusek, K., 1986, Network formation and curing of epoxy resins. Adv. Polym. Sci. 78, 1-59.

Dusek, K., 1987, Network build-up and structure in curing of epoxy resins. Makromol. Chem. Makromol. Symp. 7, $37-53$.

Erman, B. and Flory, P. J., 1978, Theory of elasticity of polymer networks. II. The effect of geometric constraints on junctions. J. chem. Phys. 68, 5363-5369.

Erman, B. and Flory, P. J., 1982, Relationships between stress, strain and molecular constitution of polymer networks. Comparison of theory with experiments. Macromolecules 15, 806-811.

Flory, P. J., 1941a, Molecular size distribution in three dimensional polymers. I. Gelation. J. Am. Chem. Soc. 63, 3083-3090.

Flory, P. J., 1941b, Molecular size distribution in three dimensional polymers. II. Trifunctional branching units. J. Am. Chem. Soc. 63, 3091-3096.

Flory, P. J., 1941c, Molecular size distribution in three dimensional polymers. III. Tetrafunctional branching units. J. Am. Chem. Soc. 63, 3096-3100.

Flory, P. J., 1942, Constitution of three dimensional polymers and the theory of gelation. J. Am. Chem. Soc. 46, 132-197.

Flory, P. J., 1947, Molecular size distribution in three dimensional polymers. V. Post-gelation relationships. J. Am. Chem. Soc. 69, 30-35.

Flory, P. J., 1953, Principles of Polymer Chemistry. Cornell University Press, Ithaca, NY.

Flory, P. J., 1977, Theory of elasticity of polymer networks. The effect of local constraints on junctions. J. chem. Phys. $66,5720-5729$.

Flory, P. J., 1982, Elastic activity of imperfect networks. Macromolecules 15, 99-100.

Flory, P. J. and Erman, B., 1984, Silicone networks with junctions of high functionality and the theory of rubber elasticity. J. Polym. Sci. Polym. Phys. Edn 22, 49-55.

Good, I. J., 1962, Cascade theory and the molecular averages of the sol fraction. Proc. R. Soc. A272, 54-59.

Gordon, M., 1962, Good's theory of cascade processes applied to the statistics of polymer distributions. Proc. $R$. Soc. A268, 240-259.

Gordon, M. and Leonis, C., 1975a, Lauric acid/pentaerythritol monolaurate: a model melt esterification. Part 1: kinetics. J. chem. Soc. Faraday I 71, 161-177.

Gordon, M. and Leonis, C., 1975b, Lauric acid/pentaerythritol monolaurate: a model melt esterification. Part 2: statistical distribution of products. J. chem. Soc. Faraday I 71, 178-188.

Gordon, M. and Scantlebury, G. R., 1964, Non-random polycondensation: statistical theory of the substitution effect. Trans. Faraday Soc. 60, 604-621.

Gottlieb, M., Macosko, C. W., Benjamin, G. S., Meyers, K. O. and Merrill, E. W., 1981, Equilibrium modulus of model poly(dimethylsiloxane) networks. Macromolecules 14, 1039-1046.

Kuchanov, S. I., Korolev, S. V. and Slin'ko, M. G., 1983, The graph theory for a description of the configurational statistics of branched polycondensate polymers. Polym. J. 15, 775-784.

Kuchanov, S. I. and Pis'men, L. M., 1972a, Calculation of the polycondensation kinetics for monomers having reactive centres with different reactivities. Polym. Sci. USSR 14, $147-160$.

Kuchanov, S. I. and Pis'men, L. M., 1972b, Calculation of the polycondensation kinetics of monomers containing reaction centres of variable activity. Polym. Sci. USSR 14, 985-993.

Kuchanov, S. I. and Povolotskaya, Ye. S., 1982, Calculation of the gel-point for non-equilibrium polycondensation 
taking into account the "substitution effect". Polym. Sci. USSR 24, 2512-2519.

Langley, N. R., 1968, Elastically effective strand density in polymer networks. Macromolecules 1, 348-352.

Lee, K.-J. and Eichinger, B. E., 1990, Computer simulation of the structure and elasticity of polyurethane networks: 1. Polyoxypropylene tetrols and hexamethylene diisocyanate. Polymer 31, 406-413.

Macosko, C.W. and Miller, D. R., 1976, A new derivation of average molecular weights of nonlinear polymers. Macromolecules 9, 199-211.

Mikes, J, and Dusek, J., 1982, Simulation of polymer network formation by the Monte Carlo method. Macromolecules 15, 93-99.

Miller, D.R. and Macosko, C. W., 1976, A new derivation of post gel properties of network polymers. Macromolecules 9, 206-211.

Miller, D. R. and Macosko, C. W., 1980, Substitution effects in property relations for stepwise polyfunctional polymerization. Macromolecules 13, 1063-1069.

Mills, P. L., 1986a, Analysis of multiphase polycarbonate polymerization in a semibatch reactor. Chem. Engng Sci. 41, 1045-1052.

Mills, P. L., 1986b, Design of multiphase gas-liquid polymerization reactors with application to polycarbonate polymerization. Ind. Engng Chem. Proc. Des. Dev. 25, 575-584.

Mills, P. L., 1986c, Determination of polymer chain length distributions by numerical inversion of $z$-transforms. Comput. Chem. Engng 10, 399-420.

Moschiar, S. M., Piacentini, C. A. L. and Williams, R. J. J., 1988, Analysis of build-up of polyurethane networks from toluenediisocyanate and castor oil considering intramolecular reactions. Polymer 29, 914-918.

Pearson, D. S. and Graessley, W. W., 1980, Elastic properties of well-characterized ethylene-propylene copolymer networks. Macromolecules 13, 1001-1009.

Pis'men, L. M. and Kuchanov, S. I., 1971, Multifunctional polycondensation and gelation. Polym. Sci. USSR 13, 890-902.

Sarmoria, C. and Miller, D. R., 1991, Models for the first shell substitution effect in stepwise polymerization. Macromolecules $24,1833-1845$.

Scanlan, J., 1960, The effect of network fiaws on the elastic properties of vulcanizates. J. Polym. Sci. 43, 501-508.

Stockmayer, W. H., 1943, Theory of molecular size distribution and gel formation in branched-chain polymers. $J$. chem. Phys. 11, 45-55.

Stockmayer, W. H., 1944, Theory of molecular size distribution and gel formation in branched-chain polymers. II. General cross-linking. J. chem. Phys. 12, 125-131.

Treloar, L. R. G., 1975, The Physics of Rubber Elasticity, 3rd Edition. Clarendon Press, Oxford.

Tsou, A. H. and Peppas, N., 1988, Kinetic analysis of curing of tetraepoxides and diamines in the presence of etherification side reactions. J. Polym. Sci. Polym. Phys. Edn 26, 2043-2060.

Valles, E. M. and Macosko, C. W., 1979, Properties of networks formed by end linking of poly(dimethylsiloxane). Macromolecules 12, 673-679.

Váquez, 'Á., Adabbo, H. E. and Williams, R. J. J., 1984, Statistics of resols. Ind. Engng Chem. Prod. Res. Dev. 23, 375-379.

Villermaux, J. and Blavier, L., 1984, Free radical polymerization engineering-I. A new method for modeling free radical polymerizations. Chem. Engng Sci. 39, 87-89.

\section{APPENDIX A: RELATION BETWEEN DISCRETE}

TRANSFORMS OF MOLECULAR WEIGHT DISTRIBUTION AND OF NUMBER MOLECULAR WEIGHT DISTRIBUTION

The molecular weight $M[\mathrm{P}(\mathrm{a}, \mathrm{n})]$ of a molecule $\mathrm{P}(\mathrm{a}, \mathrm{n})$ containing $\left(n^{m 0}, n^{m 1}, \ldots\right)$ repeating units of subclasses $\mathrm{U}^{\mathrm{m} 0}, \mathbf{U}^{\mathrm{m} !}, \ldots$, of repeating units $\mathrm{U}^{\mathrm{m}}$, with molecular weights $M^{m 0}, M^{m 1}, \ldots$, is given by

$$
M[\mathrm{P}(\mathrm{a}, \mathrm{n})]=\sum_{m=1}^{N} \sum_{k=0}^{\infty} M^{m k} n^{m k}+\sum_{i=1}^{G} M_{i} a_{i} .
$$

The discrete transform of polymer molecular weight distribution is the function

$$
\bar{P}(\mu)=\sum_{j=0}^{\infty} \mu^{M(j)} P_{j} .
$$

The index $j$ sweeps all kinds of molecules. Taking into account (A1), this becomes

$$
\begin{aligned}
& \bar{P}(\mu)=\sum_{n^{10}=0}^{\infty} \sum_{n^{11}=0}^{\infty} \mu^{n^{10} M^{10}+n^{12} M^{11}+\cdots} \ldots \sum_{n^{* 0}=0}^{\infty} \sum_{n^{* 1}=0}^{\infty}
\end{aligned}
$$

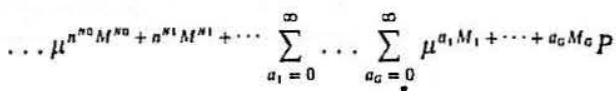

$$
\begin{aligned}
& \times\left(a_{1}, \ldots, a_{G}, n^{10}, n^{11}, \ldots, n^{N 0}, n^{N 1} \ldots\right) \text {. }
\end{aligned}
$$

Since the probabilities of occurrence of specified vectors of numbers of subclasses of each repeating unit follow multinomial distributions, the mole concentration of polymer with given numbers of end-groups $a_{1}, \ldots, a_{G}$ and of classes of repeating units $n^{10}, n^{11}, \ldots, n^{N 0}, \ldots$ is given by

$$
\begin{aligned}
P\left(a_{1}, \ldots, a_{G}, n^{10}, n^{11}, \ldots, n^{N 0}, \ldots\right) \\
=\left(\begin{array}{c}
n^{1} \\
n^{10} n^{11} n^{12} \ldots
\end{array}\right) \cdots\left(\begin{array}{c}
n^{N} \\
n^{N 0} n^{N 1} n^{N 2} \ldots
\end{array}\right) \\
\quad \times\left(\Omega^{10}\right)^{n^{10}}\left(\Omega^{11}\right)^{n^{11}} \ldots\left(\Omega^{N 0}\right)^{n^{N 0}}\left(\Omega^{N 1}\right)^{n^{N 1}} \\
\quad \ldots P\left(a_{1}, \ldots, a_{G}, n^{1}, n^{2}, \ldots, n^{N}\right) .
\end{aligned}
$$

Equation (2) is now obtained by substitution of eq. (A4) into eq. (A3), with simplification of the powers of $n^{1}, n^{2}, \ldots, n^{N}$ :

$$
\begin{aligned}
& \bar{P}(\mu)=\sum_{a_{1}=0}^{\infty} \ldots \sum_{a_{G}=0}^{\infty} \mu^{a_{3} M_{1}+\cdots+a_{G} M_{G}} \sum_{n^{i}=0}^{\infty} \\
& \ldots \sum_{n^{*}=0}^{\infty} P\left(a_{1}, \ldots, a_{G}, n^{1}, n^{2}, \ldots, n^{N}\right) \\
& \times \sum_{n^{10}=0}^{\infty} \sum_{n^{11}=0}^{\infty} \ldots\left(\mu^{M f^{10}} \Omega^{10}\right)^{n^{10}}\left(\mu^{M^{11}} \Omega^{11}\right)^{n^{11}} \\
& \cdots\left(\begin{array}{c}
n^{1} \\
n^{10} n^{11} n^{12} \ldots
\end{array}\right) \ldots \sum_{n^{N 0}=0}^{\infty} \sum_{n^{N 1}=0}^{\infty}\left(\mu^{M^{N 0}} \Omega^{N 0}\right)^{n^{N 0}} \\
& \times\left(\mu^{\mathrm{A}^{N_{1}}} \Omega^{N 1}\right)^{n^{N_{1}}} \ldots\left(\begin{array}{c}
n^{N} \\
n^{N 0} n^{N 1} n^{N 2} \ldots
\end{array}\right) \\
& =\sum_{a_{1}=0}^{\infty} \ldots \sum_{a_{G}=0}^{\infty} \mu^{a_{1} M_{1}+\cdots+a_{G} M_{G}} \sum_{n^{\prime}=0}^{\infty} \\
& \times\left(\mu^{M M^{10}} \Omega^{10}+\mu^{M^{11}} \Omega^{11}+\cdots\right)^{n^{\prime}} \\
& \cdots \sum_{n^{n}=0}^{\infty}\left(\mu^{M^{N 0}} \Omega^{N 0}+\mu^{M^{N_{1}}} \Omega^{N_{1}}+\cdots\right)^{n^{n}} \\
& \times P\left(a_{1}, \ldots, a_{G}, n^{1}, n^{2}, \ldots, n^{N}\right) \\
& =\bar{P}\left(\mu^{M_{1}}, \ldots, \mu^{M_{c}}, \mu^{M^{10}} \Omega^{10}+\mu^{M^{11}} \Omega^{11}\right. \\
& \left.+\ldots, \mu^{M^{N 0}} \Omega^{N 0}+\mu^{M^{N 1}} \Omega^{N 1}+\cdots\right) \\
& =\bar{P}\left[\mu^{M_{1}}, \ldots, \mu^{M_{c}}, \bar{\Omega}^{1}(\mu), \ldots, \bar{\Omega}^{N}(\mu)\right]
\end{aligned}
$$

in which

$$
\bar{\Omega}^{m}(\mu)=\mu^{M^{-0} 0} \Omega^{m 0}+\mu^{M^{m-1}} \Omega^{m 1}+\cdots=\sum_{j=0}^{\infty} \mu^{M^{m j}} \Omega^{m j} .
$$

APPENDIX B: KINETIC SCHEME AND STOICHIOMETRIC COEFFICIENTS IN THE RADICAL COPOLYMERIZATION OF $N$ VINYL COMPOUNDS

Tables 5-12 summarize the description of a radical copolymerization of an arbitrary number of monomers, ac- 
Table 5. Description of reactive groups

\begin{tabular}{|c|c|c|}
\hline Group $\mathbf{A}_{\mathbf{i}}$ & Description & Index $i$ \\
\hline$B_{k}$ & Monomer, containing $f_{k}$ double bonds & $k$ \\
\hline$C_{k}$ & $\begin{array}{l}\text { Set of } f_{k} \text { double bonds in a repeating unit coming from } \\
\text { a monomer which has undergone a transfer reaction }\end{array}$ & $k+N$ \\
\hline $\begin{array}{l}D_{k} \\
E_{k}\end{array}$ & $\begin{array}{l}\text { Pendant double bond coming from } \mathrm{B}_{\mathrm{k}} \\
\text { Intact polymerized fragment coming from monomer }\end{array}$ & $\begin{array}{l}k+2 N \\
k+3 N\end{array}$ \\
\hline $\mathbf{R}_{0 \mathrm{k}}^{1}$ & Radical formed by addition of a primary radical to $B_{k}$ & $k+4 N$ \\
\hline $\mathbf{R}_{0 \mathrm{k}}^{2}$ & Radical formed by transfer to monomer $\mathbf{B}_{\mathbf{k}}$ & $k+5 N$ \\
\hline $\mathbf{R}_{0 \mathrm{k}}^{3}$ & Radical site formed by transfer to polymer in a site $E_{k}$ & $k+6 N$ \\
\hline $\begin{array}{l}\mathbf{R}_{\mathrm{k} \mid}^{1} \\
\mathbf{I}\end{array}$ & $\begin{array}{l}\text { Radical ending by units coming from } \mathbf{B}_{\mathbf{k}} \text { and } \mathbf{B}_{\mathbf{l}} \\
\text { Initiator }\end{array}$ & $\begin{array}{l}(k+6) N+l \\
N^{2}+7 N+1\end{array}$ \\
\hline$I^{*}$ & Primary radical & $N^{2}+7 N+2$ \\
\hline $\mathbf{S}$ & Solvent or chainlength regulator & $N^{2}+7 N+3$ \\
\hline
\end{tabular}

Table 6. Stoichiometry of initiation reactions

\begin{tabular}{|c|c|c|c|}
\hline Reaction & $i$ & $k_{i}^{*}$ & $\bar{v}_{i}^{*}$ \\
\hline $\mathrm{I} \rightarrow \mathrm{I}^{*}$ & $N^{2}+7 N+1$ & $2 \phi k_{d}$ & $\frac{\sigma_{i+1}}{\sigma_{i}}$ \\
\hline $\mathbf{I}^{*}+\mathbf{B}_{\mathbf{k}} \rightarrow \mathbf{R}_{0 \mathrm{k}}^{1}+\left(f_{k}-1\right) \mathbf{D}_{\mathbf{k}}$ & $N^{2}+7 N+2$ & $f_{k} k_{p_{t}}^{0} A_{k}$ & $\frac{1}{\sigma_{i}}$ \\
\hline $\mathbf{I}^{*}+\mathbf{C}_{\mathrm{k}} \rightarrow \mathbf{R}_{0 \mathrm{k}}^{1}+\left(f_{\mathrm{k}}-1\right) \mathbf{D}_{\mathrm{k}}$ & $N^{2}+7 N+2$ & $f_{k} k_{p_{t}}^{0} A_{k}$ & $\frac{1}{\sigma_{i}}$ \\
\hline$I^{*}+D_{k} \rightarrow R_{0 k}^{1}$ & $N^{2}+7 N+2$ & $k_{p t}^{1} A_{k+4 N}$ & $\frac{1}{\sigma_{i}}$ \\
\hline $\mathbf{B}_{\mathbf{k}}+\mathbf{I}^{*} \rightarrow \mathbf{R}_{0 \mathbf{k}}^{1}+\left(f_{k}-1\right) \mathbf{D}_{\mathbf{k}}$ & $k$ & $f_{k} k_{p_{4}}^{0} A_{N^{2}+7 N+2}$ & $\frac{\sigma_{i+2 N}^{f_{i-1}} \sigma_{i+4 N}}{\sigma_{i}}$ \\
\hline $\mathbf{C}_{k}+I^{*} \rightarrow \mathbf{R}_{0 \mathrm{k}}^{1}+\left(f_{k}-1\right) \mathbf{D}_{k}$ & $k+N$ & $f_{k} k_{p_{1}}^{0} A_{N^{2}}+7 N+2$ & $\frac{\sigma_{i+2 N}^{f_{i-1}} \sigma_{i+4 N}}{\sigma_{i}}$ \\
\hline$D_{k}+I^{*} \rightarrow R_{0 k}^{\prime}$ & $k+2 N$ & $k_{P_{1}}^{1} A_{N^{2}+7 N+2}$ & $\frac{\sigma_{i+4 N}}{\sigma_{i}}$ \\
\hline
\end{tabular}

Table 7. Stoichiometry of propagation reactions

\begin{tabular}{|c|c|c|c|c|c|}
\hline Reaction & $i$ & $j$ & $k_{i j}$ & $\bar{v}_{i j}$ & $\bar{v}_{j i}$ \\
\hline $\mathbf{p}+\mathbf{p}^{1}+f-\mathbf{p}^{1}+\mathbf{F}$ & $k$ & $1+61 N+m$ & $f, k^{1}$ & $\sigma_{i+2 N}^{f_{i-1}} \sigma_{(m+6) N+k}$ & $\sigma_{m+3 N}$ \\
\hline $\mathbf{B}_{\mathrm{k}}+\mathbf{R}_{\mathrm{lm}} \rightarrow\left(j_{k}-1\right) \mathbf{D}_{\mathrm{k}}+\mathbf{K}_{\mathrm{mik}}+\mathbf{E}_{\mathrm{m}}$ & $\kappa$ & $(l+0) N+m$ & & $\begin{array}{c}\sigma_{i} \\
\sigma_{i+2 N}^{f_{i}-1} \sigma_{\{m+6 ! N+k}\end{array}$ & $\begin{array}{c}\sigma_{j} \\
\sigma_{m+3 N}\end{array}$ \\
\hline$B_{k}+R_{0 m}^{1} \rightarrow\left(f_{k}-1\right) D_{k}+R_{m k}^{\prime}+E_{m}$ & $k$ & $m+4 N$ & $f_{k} k_{p_{\text {nat }}}^{1}$ & $\frac{\sigma_{i}}{\sigma_{i}}$ & $\sigma_{j}$ \\
\hline $\mathbf{B}_{\mathrm{k}}+\mathbf{R}_{0 \mathrm{~m}}^{2} \rightarrow\left(f_{\mathrm{k}}-1\right) \mathbf{D}_{\mathrm{k}}+\mathbf{R}_{\mathrm{mk}}^{1}+\mathbf{C}_{\mathrm{m}}$ & $k$ & $m+5 N$ & $f_{k} k_{\text {reat }}^{1}$ & $\frac{\sigma_{i+2 N}^{j i-1} \sigma_{(m+6) N+k}}{\sigma_{i}}$ & $\frac{\sigma_{m+N}}{\sigma_{j}}$ \\
\hline $\mathbf{B}_{\mathbf{k}}+\mathbf{R}_{0 \mathrm{~m}}^{3} \rightarrow\left(f_{k}-1\right) \mathbf{D}_{\mathrm{k}}+\mathbf{R}_{\mathrm{mk}}^{1}+\mathbf{E}_{\mathrm{m}}$ & $k$ & $m+6 N$ & $f_{k} k_{p_{0+n}}^{1}$ & $\frac{\sigma_{i+2 N}^{f_{i-1}} \sigma_{(m+6) N+k}}{\sigma_{i}}$ & $\frac{1}{\sigma_{j}}$ \\
\hline $\mathbf{C}_{\mathrm{k}}+\mathbf{R}_{\mathrm{lm}}^{1} \rightarrow\left(f_{k}-1\right) \mathbf{D}_{\mathrm{k}}+\mathbf{R}_{\mathrm{mk}}^{1}+\mathbf{E}_{\mathrm{m}}$ & $k+N$ & $(l+6) N+m$ & $f_{k} k_{p_{\text {int }}}^{1}$ & $\frac{\sigma_{i+2 N}^{j_{i}-1} \sigma_{(m+6) N+k}}{\sigma_{i}}$ & $\frac{\sigma_{m+3 N}}{\sigma_{j}}$ \\
\hline $\mathbf{C}_{\mathrm{k}}+\mathbf{R}_{0 \mathrm{~m}}^{1} \rightarrow\left(f_{\mathrm{k}}-1\right) \mathbf{D}_{\mathrm{k}}+\mathbf{R}_{\mathrm{mk}}^{1}+\mathbf{E}_{\mathrm{m}}$ & $k+N$ & $m+4 N$ & $f_{k} k_{\text {pant }}^{1}$ & $\frac{\sigma_{i+2 N}^{f_{i-1}} \sigma_{(m+6) N+k}}{\sigma_{i}}$ & $\frac{\sigma_{m+3 N}}{\sigma_{j}}$ \\
\hline$C_{k}+R_{0 m}^{2} \rightarrow\left(f_{k}-1\right) D_{k}+R_{m k}^{1}+C_{m}$ & $k+N$ & $m+5 N$ & $f_{k} k_{\text {pant }}^{1}$ & $\frac{\sigma_{i+2 N}^{N-1} \sigma_{(m+' 6) N+k}}{\sigma_{i}}$ & $\frac{\sigma_{m+N}}{\sigma_{j}}$ \\
\hline $\mathbf{C}_{\mathbf{k}}+\mathbf{R}_{0 \mathrm{~m}}^{3} \rightarrow\left(f_{\mathrm{k}}-1\right) \mathbf{D}_{\mathbf{k}}+\mathbf{R}_{\mathrm{mk}}^{1}$ & $k+N$ & $m+6 N$ & $f_{\mathrm{k}} k_{p_{0 \mathrm{nat}}}^{1}$ & $\frac{\sigma_{i+2 N}^{j_{i+1}} \sigma_{(m+6) N+k}}{\sigma_{i}}$ & \\
\hline $\mathrm{D}_{\mathrm{k}}+\mathrm{R}_{\mathrm{lm}}^{1} \rightarrow \mathrm{R}_{\mathrm{mk}}^{1}+\mathrm{E}_{\mathrm{m}}$ & $k+2 N$ & $(l+6) N+m$ & $k_{p_{0 a t}}^{2}$ & $\frac{\sigma_{(m+6) N+k}}{\sigma_{i}}$ & $\frac{\sigma_{m+3 N}}{\sigma_{j}}$ \\
\hline $\mathrm{D}_{\mathrm{k}}+\mathrm{R}_{0 \mathrm{~m}}^{1} \rightarrow \mathrm{R}_{\mathrm{mk}}^{1}+\mathrm{E}_{\mathrm{m}}$ & $k+2 N$ & $m+4 N$ & $k_{p_{a+\text { at }}}^{2}$ & $\frac{\sigma_{(m+6) N+k}}{\sigma_{t}}$ & $\frac{\sigma_{m+3 N}}{\sigma_{j}}$ \\
\hline$D_{k}+R_{0 m}^{2} \rightarrow R_{m k}^{1}+C_{m}$ & $k+2 N$ & $m+5 N$ & $k_{p_{001}}^{2}$ & $\frac{\sigma_{(m+6) N+k}}{\sigma_{i}}$ & $\frac{\sigma_{m+N}}{\sigma_{j}}$ \\
\hline $\mathbf{D}_{\mathrm{k}}+\mathbf{R}_{0 \mathrm{~m}}^{3} \rightarrow \mathbf{R}_{\mathrm{mk}}^{1}$ & $k+2 N$ & $m+6 N$ & $k_{p_{00 t}}^{2}$ & $\frac{\sigma_{(m+6) N+k}}{\sigma_{i}}$ & $\frac{1}{\sigma_{j}}$ \\
\hline
\end{tabular}


Table 8. Stoichiometry of terminations by combination

\begin{tabular}{|c|c|c|c|c|c|}
\hline Reaction & $i$ & $j$ & $k_{i j}$ & $\bar{v}_{i j}$ & $\bar{v}_{j i}$ \\
\hline $\mathbf{R}_{\mathrm{kl}}^{1}+\mathbf{R}_{\mathrm{mn}}^{\prime} \rightarrow$ products & $(k+6) N+l$ & $(m+6) N+n$ & $k_{k_{k, k}}^{11}$ & $\frac{1}{\sigma_{i}}$ & $\frac{1}{\sigma_{j}}$ \\
\hline $\mathbf{R}_{\mathrm{kt}}^{1}+\mathbf{R}_{0 \mathrm{~m}}^{1} \rightarrow$ products & $(k+6) N+l$ & $m+4 N$ & $k_{t c_{i-1}}^{11}$ & $\frac{1}{\sigma_{i}}$ & $\frac{1}{\sigma_{j}}$ \\
\hline $\mathbf{R}_{\mathrm{kl}}^{1}+\mathbf{R}_{0 \mathrm{~m}}^{2} \rightarrow$ products & $(k+6) N+l$ & $m+5 N$ & $k_{l r_{i=}}^{12}$ & $\frac{1}{\sigma_{i}}$ & $\frac{1}{\sigma_{j}}$ \\
\hline $\mathbf{R}_{\mathrm{kl}}^{1}+\mathbf{R}_{0 \mathrm{~m}}^{3} \rightarrow$ products & $(k+6) N+!$ & $m+6 N$ & $k_{t i_{i=}}^{13}$ & $\frac{1}{\sigma_{i}}$ & $\frac{1}{\sigma_{j}}$ \\
\hline $\mathbf{R}_{0 \mathrm{~m}}^{1}+\mathbf{R}_{0 \mathrm{n}}^{1} \rightarrow$ products & $m+4 N$ & $n+4 N$ & $k_{t r \ldots .}^{11}$ & $\frac{1}{\sigma_{i}}$ & $\frac{1}{\sigma_{j}}$ \\
\hline $\mathbf{R}_{0 \mathrm{~m}}^{1}+\mathbf{R}_{0 \mathrm{n}}^{2} \rightarrow$ products & $m+4 N$ & $n+5 N$ & $k_{1 r_{-}}^{12}$ & $\frac{1}{\sigma_{i}}$ & $\frac{1}{\sigma_{j}}$ \\
\hline $\mathbf{R}_{0 m}^{1}+\mathbf{R}_{0 \mathrm{n}}^{3} \rightarrow$ products & $m+4 N$ & $n+6 N$ & $k_{1 / \ldots}^{13}$ & $\frac{1}{\sigma_{i}}$ & $\frac{1}{\sigma_{j}}$ \\
\hline $\mathbf{R}_{0 \mathrm{~m}}^{2}+\mathbf{R}_{0 \mathrm{n}}^{2} \rightarrow$ products & $m+5 N$ & $n+5 N$ & $k_{11 \ldots .}^{22}$ & $\frac{1}{\sigma_{i}}$ & $\frac{1}{\sigma_{j}}$ \\
\hline $\mathbf{R}_{0 m}^{2}+\mathbf{R}_{0 \mathrm{n}}^{3} \rightarrow$ products & $m+5 N$ & $n+6 N$ & $k_{k_{\ldots}}^{23}$ & $\frac{1}{\sigma_{i}}$ & $\frac{1}{\sigma_{j}}$ \\
\hline $\mathbf{R}_{0 \mathrm{~m}}^{3}+\mathbf{R}_{0 \mathrm{n}}^{3} \rightarrow$ products & $m+6 N$ & $n+6 N$ & $k_{1 r_{m .}}^{33}$ & $\frac{1}{\sigma_{i}}$ & $\frac{1}{\sigma_{j}}$ \\
\hline
\end{tabular}

Table 9. Stoichiometry of terminations by dismutation

\begin{tabular}{|c|c|c|c|}
\hline Reaction & $i$ & $k_{i}^{*}$ & $\bar{v}_{i}^{*}$ \\
\hline $\mathbf{R}_{\mathrm{kl}}^{\prime}+\mathbf{R}_{\mathrm{mn}}^{1} \rightarrow$ products & $(k+6) N+1$ & $k_{1 u_{t a}}^{11} A_{(m+6) N+n}$ & $\frac{1}{\sigma_{i}}$ \\
\hline $\mathbf{R}_{\mathrm{kl}}^{\prime}+\mathbf{R}_{\mathrm{lm}}^{\prime} \rightarrow$ products & $(k+6) N+1$ & $k_{t u_{m}}^{11} A_{m+4 N}$ & $\frac{1}{\sigma_{i}}$ \\
\hline $\mathbf{R}_{\mathrm{kl}}^{1}+\mathbf{R}_{0 \mathrm{~m}}^{2} \rightarrow$ products & $(k+6) N+l$ & $k_{t d_{m}}^{12} A_{m+5 N}$ & $\frac{1}{\sigma_{i}}$ \\
\hline $\mathbf{R}_{\mathrm{kl}}^{1}+\mathbf{R}_{0 \mathrm{~m}}^{3} \rightarrow$ products & $(k+6) N+1$ & $k_{t d_{m}}^{13} A_{m+6 N}$ & $\frac{1}{\sigma_{i}}$ \\
\hline $\mathbf{R}_{0 \mathrm{~m}}^{1}+\mathbf{R}_{0 \mathrm{n}}^{1} \rightarrow$ products & $m+4 N$ & $k_{r d_{m}}^{11} A_{m+4 N}$ & $\frac{1}{\sigma_{i}}$ \\
\hline $\mathbf{R}_{0 m}^{1}+\mathbf{R}_{0 \mathrm{n}}^{2} \rightarrow$ products & $m+4 N$ & $k_{t t_{m}}^{12} A_{m+5 N}$ & $\frac{1}{\sigma_{i}}$ \\
\hline $\mathbf{R}_{0 m}^{1}+\mathbf{R}_{0 \mathrm{n}}^{3} \rightarrow$ products & $m+4 N$ & $k_{t d_{m}}^{13} A_{m+6 N}$ & $\frac{1}{\sigma_{i}}$ \\
\hline $\mathbf{R}_{0 \mathrm{~m}}^{2}+\mathbf{R}_{0 \mathrm{n}}^{2} \rightarrow$ products & $m+5 N$ & $k_{t d_{m}}^{22} A_{m+5 N}$ & $\frac{1}{\sigma_{i}}$ \\
\hline $\mathbf{R}_{0 m}^{2}+\mathbf{R}_{0 \mathrm{n}}^{3} \rightarrow$ products & $m+5 N$ & $k_{t d . .}^{23} A_{m+6 N}$ & $\frac{1}{\sigma_{i}}$ \\
\hline $\mathbf{R}_{0 \mathrm{~m}}^{3}+\mathbf{R}_{0 \mathrm{n}}^{3} \rightarrow$ products & $m+6 N$ & $k_{t d_{m}}^{33} A_{m+6 N}$ & $\frac{1}{\sigma_{i}}$ \\
\hline
\end{tabular}

cording to the general approach developed in the body of this paper. The following assumptions were used:

(a) Chemical initiation by a single monofunctional initiator I;

(b) Equal reactivity of all double bonds in each monomer, changing as soon as one of these bonds has reacted, but not changing reactivity any further-this is needed for describing reactions of monovinyl and divinyl compounds; (c) No primary radical termination and no transfer to the initiator;

(d) Dependence of propagation rate constants on the chemical nature of the two units in the chain end;

(e) Dependence of termination and transfer rate constants on the chemical nature of the last units in the chain end;

These assumptions can easily be relaxed in order to accomodate more complex reaction schemes, provided that no 
Table 10. Stoichiometry of transfers to monomers

\begin{tabular}{llll}
\hline Reaction & \multicolumn{1}{c}{$i$} & \multicolumn{1}{c}{$k_{i}^{*}$} & $\bar{v}_{i}^{*}$ \\
\hline $\mathbf{B}_{\mathrm{k}}+\mathbf{R}_{\mathrm{lm}}^{1} \rightarrow \mathbf{R}_{0 \mathrm{k}}^{2}$ & $k$ & $k_{M_{\mathrm{m}}}^{1} A_{(m+6) N+n}$ & $\frac{\sigma_{i+5 N}}{\sigma_{i}}$ \\
$\mathbf{B}_{\mathrm{k}}+\mathbf{R}_{0 \mathrm{~m}}^{1} \rightarrow \mathbf{R}_{0 \mathrm{k}}^{2}$ & $k$ & $k_{M_{\mathrm{om}}}^{1} A_{m+4 N}$ & $\frac{\sigma_{i+5 N}}{\sigma_{i}}$ \\
$\mathbf{B}_{\mathrm{k}}+\mathbf{R}_{0 \mathrm{~m}}^{2} \rightarrow \mathbf{R}_{0 \mathrm{k}}^{2}$ & $k$ & $k_{M_{0 m}}^{2} A_{m+5 N}$ & $\frac{\sigma_{i+5 N}}{\sigma_{i}}$ \\
$\mathbf{B}_{\mathrm{k}}+\mathbf{R}_{0 \mathrm{~m}}^{3} \rightarrow \mathbf{R}_{0 \mathrm{k}}^{2}$ & $k$ & $k_{M_{0 m}}^{3} A_{m+6 N}$ & $\frac{\sigma_{i+5 N}}{\sigma_{i}}$ \\
$\mathbf{R}_{\mathrm{kl}}^{1}+\mathbf{B}_{\mathrm{m}} \rightarrow$ products & $(k+6) N+l$ & $k_{M_{\mathrm{m}}}^{1} A_{m}$ & $\frac{1}{\sigma_{i}}$ \\
$\mathbf{R}_{0 \mathrm{k}}^{1}+\mathbf{B}_{\mathrm{m}} \rightarrow$ products & $k+4 N$ & $k_{M_{\mathrm{m}}}^{1} A_{m}$ & $\frac{1}{\sigma_{i}}$ \\
$\mathbf{R}_{0 \mathrm{k}}^{2}+\mathbf{B}_{\mathrm{m}} \rightarrow$ products & $k+5 N$ & $k_{M_{\mathrm{m}}}^{2} A_{m}$ & $\frac{1}{\sigma_{i}}$ \\
$\mathbf{R}_{0 \mathrm{k}}^{3}+\mathbf{B}_{\mathrm{m}} \rightarrow$ products & $k+6 N$ & $k_{M_{\mathrm{im}}}^{3} A_{m}$ & $\frac{1}{\sigma_{i}}$ \\
\hline
\end{tabular}

Table 11. Transfers to polymer

\begin{tabular}{|c|c|c|c|}
\hline Reaction & $i$ & $k_{i}^{*}$ & $\bar{v}_{i}^{*}$ \\
\hline $\mathbf{E}_{\mathrm{k}}+\mathbf{R}_{\mathrm{tm}}^{1} \rightarrow \mathbf{R}_{0 \mathrm{k}}^{3}$ & $k+3 N$ & $k_{P_{m}}^{1} A_{(I+6) N+m}$ & $\frac{\sigma_{i+3 N}}{\sigma_{i}}$ \\
\hline $\mathbf{E}_{\mathrm{k}}+\mathbf{R}_{0 \mathrm{~m}}^{1} \rightarrow \mathbf{R}_{0 \mathrm{k}}^{3}$ & $k+3 N$ & $k_{P_{n t}}^{1} A_{m+4 N}$ & $\frac{\sigma_{i+3 N}}{\sigma_{i}}$ \\
\hline $\mathbf{E}_{\mathrm{k}}+\mathbf{R}_{0 \mathrm{~m}}^{2} \rightarrow \mathbf{R}_{0 \mathrm{k}}^{3}$ & $k+3 N$ & $k_{P_{m}}^{2} A_{m+5 N}$ & $\frac{\sigma_{i+3 N}}{\sigma_{i}}$ \\
\hline $\mathrm{E}_{\mathrm{k}}+\mathbf{R}_{\mathrm{0m}}^{3} \rightarrow \mathrm{R}_{0 \mathrm{k}}^{2}$ & $k+3 N$ & $k_{P_{m}}^{3} A_{m+6 N}$ & $\frac{\sigma_{i+3 N}}{\sigma_{i}}$ \\
\hline $\mathbf{R}_{\mathbf{k l}}^{1}+\mathbf{E}_{\mathrm{m}} \rightarrow$ products & $(k+6) N+l$ & $k_{P_{m}}^{1} A_{m+3 N}$ & $\frac{1}{\sigma_{i}}$ \\
\hline $\mathbf{R}_{0 \mathrm{k}}^{1}+\mathbf{E}_{\mathrm{m}} \rightarrow$ products & $k+4 N$ & $k_{P_{i m}}^{1} A_{m+3 N}$ & $\frac{1}{\sigma_{i}}$ \\
\hline $\mathbf{R}_{0 \mathrm{k}}^{2}+\mathbf{E}_{\mathrm{m}} \rightarrow$ products & $k+5 N$ & $k_{P_{i m}}^{2} A_{m+3 N}$ & $\frac{1}{\sigma_{i}}$ \\
\hline $\mathbf{R}_{0 \mathrm{k}}^{3}+\mathbf{E}_{\mathrm{m}} \rightarrow$ products & $k+6 N$ & $k_{P_{l m}}^{3} A_{m+3 N}$ & $\frac{1}{\sigma_{i}}$ \\
\hline
\end{tabular}

Table 12. Transfers to solvent

\begin{tabular}{|c|c|c|c|}
\hline Reaction & $i$ & $k_{i}^{*}$ & $\bar{v}_{i}^{*}$ \\
\hline $\mathbf{R}_{\mathrm{kl}}^{1}+\mathrm{S} \rightarrow \mathrm{I}^{*}$ & $(k+6) N+l$ & $k_{s_{1}}^{1} S$ & $\frac{1}{\sigma_{i}}$ \\
\hline $\mathbf{R}_{0 \mathrm{k}}^{1}+\mathrm{S} \rightarrow \mathrm{I}^{*}$ & $k+4 N$ & $k_{S_{k}}^{1} S$ & $\frac{1}{\sigma_{i}}$ \\
\hline $\mathbf{R}_{0 k}^{2}+S \rightarrow I^{*}$ & $k+5 N$ & $k_{S_{k}}^{2} S$ & $\frac{1}{\sigma_{i}}$ \\
\hline $\mathbf{R}_{0 \mathrm{k}}^{3}+\mathrm{S} \rightarrow \mathrm{I}^{*}$ & $k+6 N$ & $k_{S_{1}}^{3} S$ & $\frac{1}{\sigma_{i}}$ \\
\hline $\mathbf{R}_{\mathrm{kl}}^{\mathrm{l}}+\mathrm{S} \rightarrow \mathrm{I}^{*}$ & $N^{2}+7 N+2$ & $k_{S_{t}}^{1} S$ & $\sigma_{i}$ \\
\hline $\mathbf{R}_{0 \mathrm{k}}^{1}+\mathbf{S} \rightarrow \mathbf{I}^{*}$ & $N^{2}+7 N+2$ & $k_{S_{x}}^{1} S$ & $\sigma_{i}$ \\
\hline $\mathbf{R}_{0 \mathrm{k}}^{2}+\mathbf{S} \rightarrow \mathbf{I}^{*}$ & $N^{2}+7 N+2$ & $k_{S_{t}}^{2} S$ & $\sigma_{i}$ \\
\hline $\mathbf{R}_{0 \mathrm{k}}^{3}+\mathrm{S} \rightarrow \mathbf{I}^{*}$ & $N^{2}+7 N+2$ & $k_{S_{4}}^{3} S$ & $\sigma_{i}$ \\
\hline
\end{tabular}

molecular break-up occurs in the middle of a chain (such as $\beta$-scission of radicals).

There was no attempt to follow the number of initiator fragments per molecule, as well as transfer reactions of those fragments, but this could also be done without much added difficulty. For this reason, primary radicals coming from the initiator or from the solvent were not distinguished.

Notice also that initiator I is taken as "polymer" species with zero repeating units and a single group I (see Table 5). Monomer $k$ is a species with a single repeating unit $\mathrm{U}_{\mathrm{k}}$ and one kind of reactive groups $\mathbf{B}_{k}$, the set of its $f_{k}$ double bonds and the remaining molecule, which can undergo transfer reactions. 